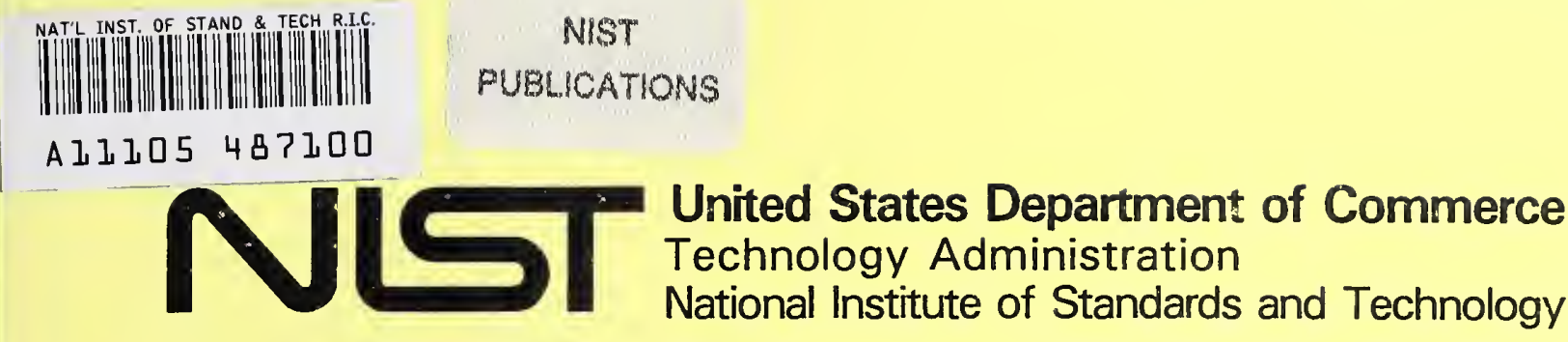

NISTIR 5075

Metrology for Radio-Frequency Technology: A Bibliography of NIST Publications

Ruth Marie Lyons, Editor

September 1998

QC

100

.056

N0.5075

1998 



\section{Metrology for Radio-Frequency Technology: A Bibliography of NIST Publications}

Ruth Marie Lyons, Editor

Radio-Frequency Technology Division

Electronics and Electrical Engineering Laboratory

National Institute of Standards and Technology

Boulder, Colorado 80303-3328

September 1998

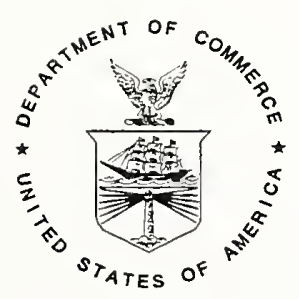

U.S. DEPARTMENT OF COMMERCE, William M. Daley, Secretary TECHNOLOGY ADMINISTRATION, Gary R. Bachula, Acting Under Secretary for Technology NATIONAL INSTITUTE OF STANDARDS AND TECHNOLOGY, Robert E. Hebner, Acting Director 



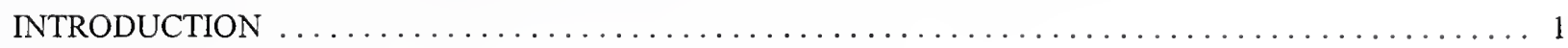

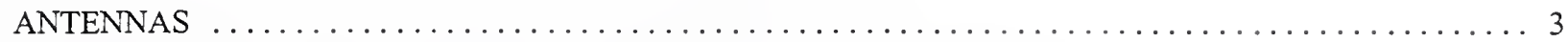

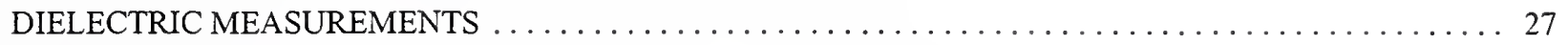

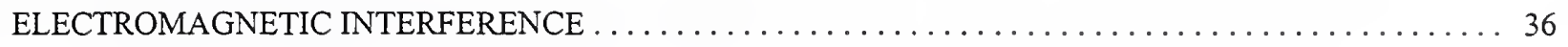

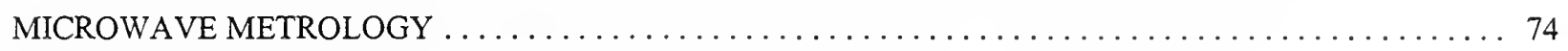

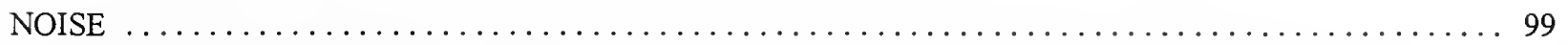

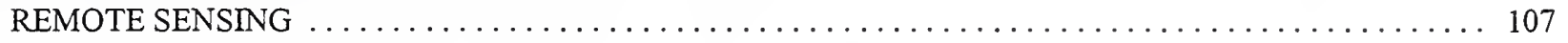

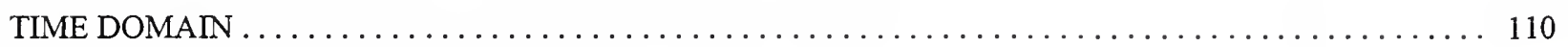

WAVEFORM METROLOGY $\ldots \ldots \ldots \ldots \ldots \ldots \ldots \ldots \ldots \ldots \ldots \ldots \ldots \ldots \ldots \ldots \ldots \ldots \ldots \ldots \ldots$

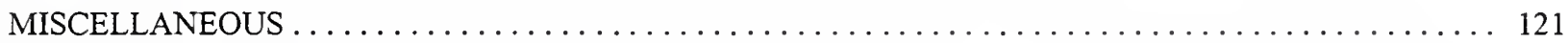

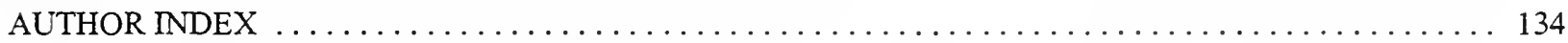





\title{
METROLOGY FOR RADIO-FREQUENCY TECHNOLOGY A BIBLIOGRAPHY OF NIST PUBLICATIONS
}

\author{
Edited by \\ Ruth Marie Lyons \\ National Institute of Standards and Technology \\ Boulder, CO 80303
}

This bibliography lists the publications by the staff of the Radio-Frequency Technology Division of the National Institute of Standards and Technology for the period January 1970 through July 1998. It supersedes NISTIR 5063, which listed the publications of the Electromagnetic Fields Division from January 1970 through July 1997. Selected earlier publications from the Division's predecessor organizations are included.

Key words: antennas; dielectric measurements; electromagnetic interference; microwave metrology; microwave power, impedance, attenuation; near-field antenna measurements; noise; nonionizing radiation; radiation hazards; standards; time domain; waveform metrology.

\section{INTRODUCTION}

The Radio-Frequency Technology Division was established in 1998 as a result of a programmatic restructuring of the former Electromagnetic Fields Division. The new name more accurately reflects the division's current technical programs and characterizes its broad mission. Programs include metrology for the fundamental microwave quantities such as power, noise, impedance, and scattering parameters; standards and metrology for wireless systems; gain, pattern, and polarization measurements for antennas; electromagnetic properties of materials; measurements for high speed microelectronics; electromagnetic field characterization; and electromagnetic compatibility.

All known work published by the Division staff from January 1970 through July 1998 ins included. Work performed by authors before joining this Division or work by authors who have left this Division has been included only when it had special significance. A miscellaneous category includes general publications. A few references are listed in more than one category where appropriate.

Several other information sources may be useful to the reader who is interested in NIST activities connected with electromagnetic metrology. Two companion bibliographies, NISTIR 5076 and NISTIR 5077, list publications of the Electromagnetic Technology Division and the Optoelectronics Division for the same period. Topics include metrology for laser systems, optical fibers, optical communication equipment, cryoelectronics, superconductors, and other unusual electrical engineering materials. An 
excellent summary of the whole field of electromagnetic metrology as it stood in 1967 was published as a special issue of the Proceedings of the IEEE (volume 55, June 1967). Advances in the following decades were described in two other special issues of the same journal (volume 66, April 1978 and volume 74, January 1986).

\section{A Note on Abbreviations}

On August 23, 1988, the National Bureau of Standards (NBS) became the National Institute of Standards and Technology (NIST); therefore, documents with either prefix are considered NIST publications. Most readers are familiar with the commonly used abbreviations for the names of the professional journals that appear in this bibliography. Some publication series are peculiar to NIST and may call for explanation. They are:

NISTIR - NIST Interagency/Internal Report

NIST TN - NIST Technical Note

NIST SP - NIST Special Publication

NIST HB - NIST Handbook

NIST JRES - NIST Journal of Research

NIST MN - NIST Monograph

\author{
NBSIR - NBS Interagency/Internal Report \\ NBS TN - NBS Technical Note \\ NBS SP - NBS Special Publication \\ NBS HB - NBS Handbook \\ NBS JRES - NBS Journal of Research \\ NBS MN - NBS Monograph
}

Purchase Procedures and Document Availability

NIST (NBS) Technical Notes, Special Publications, Handbooks, Journals of Research, and Monographs may be purchased from the U.S. Government Printing Office (GPO). The GPO website is http://www.access.gpo.gov/ and the order desk phone number is 202-512-1800. To find a GPO bookstore near you use the following website address http://www.access.gpo.gov/su_docs/sale/ abkst001.html. The Government Printing Office usually stocks these publications for only a year or two, after which they may be purchased from the National Technical Information Service whose website for information and ordering is at http://www.ntis.gov/. The phone number for ordering is $703-$ 487-4650.

Reprints of papers published in non-NIST media may be available in limited quantities from the authors. 


\section{ANTENNAS}

WITTMANN, R. C.; ALPERT, B. K.; FRANCIS, M. H.

Near-Field Antenna Measurements Using Nonideal Measurement Locations

IEEE Trans. Antenna Propagat., Vol. 46, No. 5, 716-722

May 1998

SKINNER, J. P.; KENT, B. M.; WITTMANN, R. C.; MENSA, D. L.; ANDERSH, D.

Normalization and Interpretation of Radar Images

IEEE Trans. Antenna Propagat., Vol. 46, No. 4, pp. 502-506

April 1998

SORGNIT, J.; MORA, P.; MUTH, L. A.; WITTMANN, R. C.

Uncertainty Analysis Procedures for Dynamic Radar Cross Section Measurements at the Atlantic Test

Range

NISTIR 5073

February 1998

FRANCIS, M. H.; MILLIGAN, T.

Aperture Sampling Effects in Planar Near-Field Measurements

Proc., Antenna Meas. Tech. Assoc. Symp., 17-20 November 1997, Boston, MA, pp. 79-83

November 1997

KENT, B. M.; MUTH, L.A.

Establishing a Common RCS Pange Documentation Standards Based on ANSI/NCSL Z-540-1994-1 and ISO Guide 25

Proc., Antenna Meas. Tech. Assoc. Symp., 17-20 November 1997, Boston, MA, pp. 291-296 November 1997

MUTH, L. A.; WITTMANN, R. C.; KENT, B. M.

Interlaboratory Comparisons in Radar Cross Section Measurement Assurance

Proc., Antenna Meas. Tech. Symp., 17-21 November 1997, Boston, MA, pp. 297-307

November 1997

SKINNER, J. P.; KENT, B. M.; WITTMANN, R. C.; MENSA, D. L.; ANDERSH, D.

Radar Image Normalization and Interpretation

Proc., Antenna Meas. Tech. Assoc. Symp., 17-20 November 1997, Boston, MA, pp. 303-307

November 1997

STUBENRAUCH, C. F.; MACREYNOLDS, K.; NORGARD, J. D.; SEIFERT, M.; CORMACK, R. H. Microwave Antenna Far-Field Patterns Determined from Infrared Holograms

Proc., Antenna Meas. Tech. Symp., 17-21 November 1997, Boston, MA, pp. 125-130

November 1997

FRANCIS, M. H.

Aperture Sampling Requirements

IEEE Antennas and Propagat. Magazine, Vol. 39, No. 5, pp. 76-77

October 1997

MUTH, L. A.; WITTMANN, R. C.

Calibration of Polarimetric Radar Systems

Dig., Intl. Symp. on Antennas and Propagat., 13-18 July 1997, Montreal, Canada, pp. 830-833 July 1997 
MIUTH, L. A.; WITTMANN, R. C.; KENT, B. M.

Measurement Assurance and Certification of Radar Cross Section Measurements Proc., NCSL Conf., 27-31 July 1997, Atlanta, GA, pp. 555-566 July 1997

WITTMANN, R. C.; BLACK, D. N.

Quiet-Zone Evaluation Using a Spherical Synthetic-Aperture

Dig., Intl. Symp. on Antennas and Propagat., 13-18 July 1997, Montreal, Canada, pp. 148-151

July 1997

WITTMANN, R. C.; ALPERT, B. K.; FRANCIS, M. H.

Nonideal Measurement Locations in Planar Near-Field Antenna Metrology

Dig., Intl. Symp. on Antennas and Propagat., 13-18 July 1997, Montreal, Canada, pp. $168-171$

July 1997

PRICKETT, M. J.; BLOOMFIELD, R. A.; KINZEL, G. A.; WITTMANN, R. C.; MUTH, L. A. Uncertainty Analysis for NRaD Radar Cross Section Measurements NISTIR 5061

April 1997

STUBENRAUCH, C. F.; NEWELL, A. C.; REPJAR, A. G.; MACREYNOLDS, K.; TAMURA, D. T.; HOLMLARSEN, F.; LEMANCYZYK, J.; BEHE, R.; PORTIER, G.; ZEHREN, J. C.; HOLLMANN, H.; HUNTER, J. D.; GENTLE, D. G.; DEVREEDE, J. P. M.

International Intercomparison of Horn Gain at X-Band

IEEE Trans. Antennas Propagat., Vol. 44, No. 10, pp. 1367-1374

October 1996

GUERRIERI, J. R.; MACREYNOLDS, K.; CANALES, S.; TAMURA, D. T. Mismatch Errors in Insertion-Loss Measurements Using Harmonic Mixers

Proc., Antenna Meas. Tech. Assoc. Symp., 30 Sept.-4 Oct. 1996, Seattle, WA, pp. 377-382 September 1996

MUTH, L. A.; WITTMANN, R. C.; PARNELL, W.

Polarimetric Calibration of Nonreciprocal Radar Systems

Proc., Antenna Meas. Tech. Assoc. Symp., 30 Sept.-4 Oct. 1996, Seattle, WA, pp. 389-393 September 1996

MUTH, L. A.; WITTMANN, R.C.; KENT, B.; TUTTLE, J. D.

Radar Cross Section Range Characterization

Proc., Antenna Meas. Tech. Assoc. Symp., 30 Sept.-4 Oct. 1996, Seattle, WA, pp. 267-278 September 1996

STUBENRAUCH, C. F.; MACREYNOLDS, K.; NEWELL, A. C.; CORMACK, R. H.; WILL, J. E.; NORGARD, J. D.

Phaseless Measurements of Antenna Near Fields Employing Holographic Phase Retrieval

Proc., Antenna Meas. Tech. Assoc. Symp., 30 Sept.-4 Oct. 1996, Seattle, WA, pp. 20-24 September 1996 
Antennas (cont.)

WILL, J. E.; NORGARD, J. D.; SEGA, R. M.; SEIFERT, M.; PESTA, A.; CLEARY, J.; STUBENRAUCH, C. F.; MACREYNOLDS, $\mathrm{K}$.

Antenna Near Field Phase Data from Infrared Thermograms by Fourier Iterative Plane-to-Plane Techniques

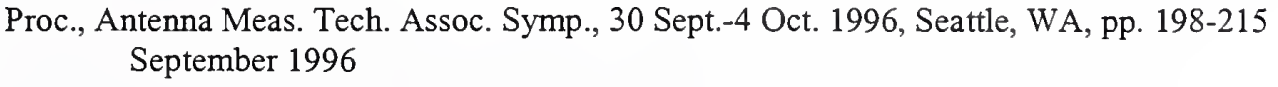

WITTMANN, R. C.; ALPERT, B. K.; FRANCIS, M. H.

Planar Near-Field Antenna Measurements Using Non-Ideal Measurement Locations

Proc., Antenna Meas. Tech. Assoc. Symp., 30 Sept.-4 Oct. 1996, Seattle, WA, pp. 74-79 September 1996

WITTMANN, R. C.; BLACK, D. N.

Antenna/RCS Range Evaluation Using a Spherical Synthetic-Aperture Radar

Proc., Antenna Meas. Tech. Assoc. Symp., 30 Sept.-4 Oct. 1996, Seattle, WA, pp. 406-410 September 1996

WILL, J. E.; NORGARD, J.D.; SEGA, R. M.; SEIFERT, M.; PESTA, A.; CLEARY, J.; STUBENRAUCH, C. F.; MACREYNOLDS, $K$.

Near-Field to Far-Field Antenna Pattern Measurements Using Infrared Imaging and Fourier Interactive Plane-to-Plane Techniques

Proc., 6th Dual-Use Technol. and Apps. Conf., 4-6 June 1996; Syracuse, NY; pp. 266-271 June 1996

WILL, J. E.; NORGARD, J. D.; STUBENRAUCH, C. F.; MACREYNOLDS, K.; SEIFERT, M.; SEGA, R. M. Phase Measurements of Electromagnetic Fields Using Infrared Imaging Techniques and Microwave Holography

Proc., 10th Intl. AeroSense Symp., 8-12 April 1996; Orlando, FL; Vol. 2766, pp. 323-333 April 1996

FRANCIS, M. H.; NEWELL, A. C.; GRIMM, K. R.; HOFFMAN, J.; SCHRANK, H. E.

Comparison of Ultralow-Sidelobe Antenna Far-Field Patterns Using the Planar Near-Field Method and the Far-Field Method

IEEE Antenna Propagat. Magazine, Vol. 37, No. 6, pp. 7-15 December 1995

FRANCIS, M. H.

A Comparison of K-Correction and Taylor-Series Correction for Probe-Position Errors in Planar NearField Scanning

Proc., Antenna Meas. Tech. Assoc. Symp., 13-17 November 1995, Williamsburg, VA, pp. 331340

November 1995

GUERRIERI, J. R.; CANALES, S.; MACREYNOLDS, K.; TAMURA, D. T.

Planar Near-Field Measurements and Microwave Holography for Measuring Aperture Distribution on a $60 \mathrm{GHz}$ Active Array Antenna

Proc., Antenna Meas. Tech. Assoc. Symp., 13-17 November 1995, Williamsburg, VA, pp. 295300

November 1995

MUTH, L. A.; LEWIS, R. L.; WITTMANN, R. C.

Polarimetric Calibration of Reciprocal-Antenna Radars

Proc., Antenna Meas. Tech. Assoc. Symp., 13-17 November 1995, Williamsburg, VA, pp. 3-8 November 1995 
LEWIS, R. L.

Spherical-Wave Source-Scattering Matrix Analysis of Antennas and Antenna-Antenna Interactions NIST TN 1373

July 1995

LEWIS, R. L.; MUTH, L. A.; WITTMANN, R. C.

Polarimetric Calibration of Reciprocal Antenna Radars: A Study of RCS Polarization Uncertainty Due to Target Depolarization

NISTIR 5033

March 1995

LEWIS, R. L.; MUTH, L. A.; WITTMANN, R. C.

RangeCAD and the NIST RCS Uncertainty Analysis

NISTIR 5022

December 1994

FRANCIS, M. H.; KREMER, D. P.; JACOBSON, M. D.; FEDOR, L. S.; HAZEN, D. A.; MADSEN, W. B. A Dual-Frequency Millimeter-Wave Radiometer Antenna for Airborme Remote Sensing of Atmosphere and Ocean

Proc., Antenna Meas. Tech. Assoc. Symp., 3-7 October, 1994 Long Beach, CA, pp. 3-9

October 1994

GUERRIERI, J. R.; TAMURA, D. T.

Effects of Microwave Absorber on Insertion-Loss Measurements

Proc., Antenna Meas. Tech. Assoc. Symp., 3-7 October 1994, Long Beach, CA, pp. 221-229

October 1994

GUERRIERI, J. R.; TAMURA, D. T.

Effects of Microwave Absorber on Insertion-Loss Measurements

Proc., Antenna Meas. Tech. Assoc. Symp., 3-7 October 1994, Long Beach, CA, pp. 221-229

October 1994

WITTMANN, R. C.; FRANCIS, M. H.; MUTH, L. A.; LEWIS, R. L.

Proposed Analysis of RCS Measurement Uncertainty

Proc., Antenna Meas. Tech. Assoc. Symp., 3-7 October, Long Beach, CA, pp. 51-57

October 1994

JACOBSON, M. D.; FEDOR, L. S.; HAZEN, D. A.; MADSEN, W. B.; FRANCIS, M. H.; KREMER, D. P. A Dual-Frequency Millimeter-Wave Radiometer Antenna for Airborne Remote Sensing of Atmosphere and Ocean

Microwave J., pp. 24-38

September 1994

MUTH, L. A.; GARY, J. M.

Accurate Computations of Radar Cross Sections of Simple Objects

Proc., MIKON '94 Microwave Conf. 30 May-2 June 1994, Ksąiż, Poland, pp. 298-302 June 1994

FRANCIS, M. H.; NEWELL, A. C.; GRIMM, K. R.; HOFFMAN, J.; SCHRANK, H. E.

Planar Near-Field Measurements of Low-Sidelobe Antennas

NIST JRES, Vol. 99, No. 2, pp. 143-167

March-April 1994 
WITTMANN, R. C.; FRANCIS, M. H.; MUTH, L. A.; LEWIS, R. L.

Proposed Uncertainty Analysis for RCS Measurements NISTIR 5019

January 1994

FRANCIS, M. H.; NEWELL, A. C.; GRIMM, K. R.; HOFFMAN, J.; SCHRANK, H. E.

Planar Near-Field Measurements of Low-Sidelobe Antennas

Proc., Antenna Meas. Tech. Assoc. Symp., 4-8 October 1993, Dallas, TX, pp. 184-189 October 1993

GUERRIERI, J. R.; KREMER, D. P.; RUSYN, T.

A New Extrapolation/Spherical/Cylindrical Measurement Facility at the National Institute of Standards and Technology

Proc., Antenna Meas. Tech. Assoc. Symp., 4-8 October 1993, Dallas, TX, pp. 343-352 October 1993

KREMER, D. P.; NEWELL, A. C.; ROSE, C.; TRABELSI, A.; PINKASI, M.

Planar Near-Field Alignment

Proc., Antenna Meas. Tech. Assoc. Symp., 4-8 October 1993, Dallas, TX, pp. 198-204

October 1993

FRANCIS, M. H.; MACREYNOLDS, K.; CANALES, S.

Dual-Port Circularly Polarized Probe Standards at the National Institute of Standards and Technology NISTIR 5007

August 1993

STUBENRAUCH, C. F.

Development of Near-Field Test Procedures for Communication Satellite

Lecture Notes, Antenna Measurement Tech. Assoc. Workshop on Communication Satellite

Antenna Meas., Ann Arbor, MI, July 2, 1993

July 1993

FRANCIS, M. H.; REPJAR, A. G.; KREMER, D. P.

A Certification Plan for a Planar Near-Field Range Used for High-Performance Phased-Array Testing Proc., Antenna Meas. Tech. Assoc. Symp., 19-23 October 1992, Columbus, OH, pp. 8-24 through 8-30

October 1992

GUERRIERI, J. R.; TAMURA, D. T.; REPJAR, A. G.

Accurate Planar Near-Field Probe Correction Using Dual-Port Circularly-Polarlized Probes

Proc., Antenna Meas. Tech. Assoc. Symp., 19-23 October 1992, Columbus, OH, pp. 10-17 through $10-25$

October 1992

MACREYNOLDS, K.; REPJAR, A. G.; KREMER, D. P.; CANALES, S.

Phased Array Testing and Diagnostics Using Planar Near-Field Scanning

Proc., Antenna Meas. Tech. Assoc. Symp., 19-23 October 1992, Columbus, OH, pp. 8-8 through 8-13

October 1992

WITTMANN, R. C.; NEWELL, A. C.; STUBENRAUCH, C. F.; MACREYNOLDS, K.; FRANCIS, M. H. Simulation of the Merged-Spectrum Techniques for Aligning Planar Phased-Array Antennas, Part I NISTIR 3981

October 1992 
FRANCIS, M. H.; REPJAR, A. G.; KREMER, D. P.

A Certification Plan for a Planar Near-Field Range Used for High-Performance Phased-Array Testing NISTIR 3991

July 1992

MUTH, L. A.

Probe-Position Error Correction in Planar Near-Field Measurement at $60 \mathrm{GHz}$ : Experimental

Verification

NIST JRES, Vol. 97, No. 2, pp. 273-297

March-April 1992

WITTMANN, R. C.

Probe-Corrected Spherical Near-Field Scanning Theory in Acoustics

IEEE Trans. Instrum. Meas., Vol. IM-41, No. 1, pp. 17-21

February 1992

FRANCIS, M. H.; WITTMANN, R. C.

Swept Frequency Gain Measurements from 33-50 GHz at the National Institute of Standards and Technology

Proc., Antenna Meas. Tech. Assoc.Symp., 7-11 October 1991, Boulder, CO, pp. 1-3 through 1-6 October 1991

REPJAR, A. G.; GUERRIERI, J. R.; KREMER, D. P.; CANALES, S.

Determining Faults on a Flat Phased Array Antenna Using Planar Near-Field Techniques

Proc., Antenna Meas. Tech. Assoc.Symp., 7-11 October 1991, Boulder, CO, pp. 8-11 through

$8-19$

October 1991

WITTMANN, R. C.; YAGHJIAN, A. D.

Spherical-Wave Expansions of Piston-Radiator Fields

J. Acoust. Soc. Amer., Vol. 30, No. 3, pp. 1647-1655

September 1991

MUTH, L. A.

General Analytic Correction for Probe-Position Errors in Spherical Near-Field Measurements

NIST JRES, Vol. 96, No. 4, pp. 391-410

July-August 1991

MUTH, L. A.; LEWIS, R. L.

Personal Computer Codes for Analysis of Planar Near Fields

NISTIR 3970

June 1991

ESTIN, A. J.; JANEZIC, M. D.

Improvements in Dielectric Measurements with a Resonant Cavity

Proc., IEEE Instrum. Meas. Technol. Conf., 14-16 May 1991, Atlanta, GA, pp. 573-579

May 1991

WITTMANN, R. C.

Probe-Corrected Spherical Near-Field Scanning Theory in Acoustics

Proc., IEEE Instrum. Meas. Technol. Conf., 14-16 May 1991, Atlanta, GA, pp. 283-286

May 1991 
MUTH, L. A.

Analytic Correction for Probe-Position Errors in Spherical Near-Field Measurements

Proc., 7th Intl. Conf. on Antennas and Propagat., 15-18 April 1991, York, England, pp. $762-765$

April 1991

MUTH, L. A.; LEWIS, R. L.

A General Technique to Correct Probe Position Errors in Planar Near-Field Measurements to Arbitrary Accuracy

IEEE Trans. Antennas Propagat., Vol. 38, No. 12, pp. 1925-1932

December 1990

FRANCIS, M. H.; MACREYNOLDS, $K$.

Evaluation of Dual-Port Circularly Polarized Probes for Planar Near-Field Measurements

Proc., Antenna Meas. Tech. Assoc. Symp., 8-11 October 1990, Philadelphia, PA, pp. 13-3

through 13-8

October 1990

MUTH, L. A.; NEWELL, A. C.; LEWIS, R. L.; CANALES, S.; KREMER, D. P.

Experimental and Theoretical Probe Position Error Correction in Near-Field Antenna Measurements Proc., Antenna Meas. Tech. Assoc. Symp., 8-11 October 1990, Philadelphia, PA, pp. 13-27 through 13-30

October 1990

KREMER, D. P.; NEWELL, A. C.

Alignment Fixture for Millimeter Waveguide

IEEE Trans. Antennas and Propagat., Vol. 32, No. 3, pp. 45-48

June 1990

WITTMANN, R. C.

Spherical Near-Field Scanning: Determining the Incident Field Near a Rotatable Probe

Proc., IEEE Intl. Symp. Antenna Propagat. Soc., 7-11 May 1990, Dallas, TX, pp. 224-227

May 1990

HILL, D. A.; EHRET, R. L.

Near-Field Gain of Pyramidal Horns from 18 to $40 \mathrm{GHz}$

NISTIR 89-3924

November 1989

FRANCIS, M. H.

Antenna Far-Field Pattern Accuracies at Millimeter Wave Frequencies Using the Planar Near-Field Technique

Proc., Antenna Meas. Tech. Assoc. Symp., 9-13 October 1989, Monterey, CA, pp. 11-16

through 11-21

October 1989

GUERRIERI, J. R.; KREMER, D. P.

Automated Multi-Axis Motor Controller and Data Acquisition System for Near-Field Scanners

Proc., Antenna Meas. Tech. Assoc. Symp., 9-13 October 1989, Monterey, CA, pp. 12-24

through 12-28

October 1989 
KREMER, D. P.; NEWELL, A. C.; AGEE, D. A.

Absorber Characterization

Proc., Antenna Meas. Tech. Assoc. Symp. 9-13 October 1989, Monterey, CA, pp. 13-7 through 13-11.

October 1989

MUTH, L. A.; LEWIS, R. L.

Planar Near-Field Codes for Personal Computers

NISTIR 89-3929

October 1989

NEWELL, A. C.; GUERRIERI, J. R.; PERSINGER, R. R.; STILES, J. A.; MCFARLANE, E. J.

Comparison of Antenna Boresight Measurements Between Near-Field and Far-Field Ranges

Proc., Antenna Meas. Tech. Assoc. Symp., 9-13 October 1989, Monterey, CA, pp. 1-24 through $1-29$

October 1989

NEWELL, A. C.; KREMER, D. P.; GUERRIERI, J. R.

Improvements in Polarization Measurements of Circularly Polarized Antennas

Proc., Antenna Meas. Tech. Assoc. Symp., 9-13 October 1989, Monterey, CA, pp. 1-30 through $1-36$

October 1989

\section{MUTH, L. A.; LEWIS, R. L.}

An Iterative Technique to Correct Probe Position Errors in Planar Near-Field to Far-Field Transformations

Proc., 1989 Intl. Symp. on Antennas and Propagat., 22-25 August 1989, Nagoya, Japan, pp. 901-904

August 1989

WITTMANN, R. C.

Comments on Spherical Near-Field Antenna Measurements

Review for Antenna Propagat. Soc. Newsletter, Vol. 31, No. 3, pp. 35-36

June 1989

HILL, D. A.

Antennas for Geophysical Applications-Antenna Handbook: Theory, Applications and Design (Chapter 3),

U. T. Lo and S. W. Lee, eds., Van Nostrand Reinhold Co., pp. 23-1 through 23-6 1988

MUTH, L. A.; LEWIS, R. L.

An Iterative Technique to Correct Probe Position Errors in Planar Near-Field to Far-Field

Transformations

NISTTN 1323

October 1988

FRANCIS, M. H.; REPJAR, A. G.; KREMER, D. P.

Antenna Measurements for Millimeter Waves at the National Bureau of Standards

Proc., Antenna Meas. Tech. Assoc., 12-16 September 1988, Atlanta, GA, pp. 13-13 through 13-17

September 1988 
Antennas (cont.)

KREMER, D. P.; REPJAR, A. G.

Antenna Calibrating Standards Using CW and Pulsed-CW Measurements and the Planar Near-Field Method

Proc., Antenna Meas. Tech. Assoc., 12-16 September 1988, Atlanta, GA, pp. 13-21 through 13-29

September 1988

NEWELL, A. C.

Development of Near-Field Test Procedures for Communication Satellite Antennas, Phase I, Part 2

NBSIR 87-3081

August 1988

WITTMANN, R. C.

Spherical Wave Operators and the Translation Formulas

IEEE Trans. Antennas Propagat., Vol. 36, No. 8, pp. 1078-1087

August 1988

BAIRD, R. C.; NEWELL, A. C.; STUBENRAUCH, C. F.

A Brief History of Near-Field Measurements of Antennas at the National Bureau of Standards

IEEE Trans. Antennas Propagat., Spec. Issue on Near-Field Scanning Technology, Vol. 36, No.

6, pp. 727-733

June 1988

LEWIS, R. L.; NEWELL, A. C.

Efficient and Accurate Method for Calculating and Representing Power Density in the Near Zone of Microwave Antennas

IEEE Trans. Antennas Propagat., Spec. Issue on Near-Field Scanning Technology, Vol. 36, No.

6, pp. 890-891

June 1988

NEWELL, A. C.; STUBENRAUCH, C. F.

Effect of Random Errors in Planar Near-Field Measurement

IEEE Trans. Antennas Propagat., Spec. Issue on Near-Field Scanning Technology, Vol. 36, No.

6, pp. 769-773

June 1988

NEWELL, A. C.

Improved Polarization Measurements Using a Modified Three Antenna Technique

IEEE Trans. Antennas Propagat., Spec. Issue on Near-Field Scanning Technology, Vol. 36, No.

6, pp. 852-854

June 1988

NEWELL, A. C.

Error Analysis Techniques for Planar Near-Field Measurements

IEEE Trans. Antennas Propagat., Spec. Issue on Near-Field Scanning Technology, Vol. 36, No.

6, pp. 754-768

June 1988

REPJAR, A. G.; NEWELL, A. C.; FRANCIS, M. H.

Accurate Determination of Planar Near-Field Correction Parameters for Linearly Polarized Probes

IEEE Trans. Antenna Propagat., Spec. Issue on Near-Field Meas., Vol. 36, No. 6, pp. 855-868

June 1988 
MUTH, L. A.

Displacement Errors in Antenna Near-Field Measurements and Their Effect on the Far Field IEEE Trans. Antennas Propagat., Vol. 36, No. 5, pp. 581-591 May 1988

FRANCIS, M. H.; STUBENRAUCH, C. F. Comparison of Measured and Calculated Antenna Side Lobe Coupling Loss in the Near Field Using Approximate Far-Field Data IEEE Trans. Antennas Propagat., Vol. 36, No. 3, pp. 438-441 March 1988

LEWIS, R. L.

Spherical-Wave Source-Scattering Matrix Analysis of Coupled Antennas; A General System Two-Port Solution

IEEE Trans. Antennas Propagat., Vol. AP-35, No. 12, pp. 1375-1380

December 1987

LEWIS, R. L.; WITTMANN, R. C.

Improved Spherical and Hemispherical Scanning Algorithms

IEEE Trans. Antennas Propagat., Vol. AP-35, No. 12, pp. 1381-1388

December 1987

HILL, D. A.; FRANCIS, M. H.

Out-of-Band Response of Antenna Arrays

IEEE Trans. Electromagn. Compat., Vol. EMC-29, No. 4, pp. 282-288 November 1987

FRANCIS, M. H.; HILL, D. A.

Out-of-Band Response of Array Antennas

Proc., Antenna Meas. Tech. Assoc., 28 September-2 October 1987, Seattle, WA, pp. 14-19 September 1987

MUTH, L. A.

Experimental Study of Interpanel Interactions at $3.3 \mathrm{GHz}$

Proc., Antenna Meas. Tech. Assoc., 28 September-2 October 1987, Seattle, WA, pp. 25-29 September 1987

HILL, D. A.; FRANCIS, M. H.

Out-of-Band Response of Antenna Arrays Proc., 1987 IEEE Intl. Symp. Electromagn. Compat., 25-27 August 1987, Atlanta, GA, pp. $435-438$

August 1987

REPJAR, A. G.; NEWELL, A. C.; TAMURA, D. T.

Extrapolation Range Measurements for Determining Antenna Gain and Polarization NBS TN 1311

August 1987

HILL, D. A.; CAVCEY, K. H.

Coupling Between Two Antennas Separated by a Planar Interface

IEEE Trans. Geosci. Remote Sensing, Vol. GE-25, No. 4, pp. 422-431

July 1987 
DAYWITT, W. C.

A Simple Technique for Determining Joint Losses on a Coaxial Line from Swept-Frequency Reflection Data

IEEE Trans. Instrum. Meas., Vol. IM-36, pp. 468-473 June 1987

FITZGERRELL, R. G.

Three PC-Computer Programs for Antenna Calculations Primarily for Use Below $1000 \mathrm{MHz}$ IEEE Antenna Propagat. Soc. Newslett., Vol. 29, No. 3, pp. 30-32 June 1987

KANDA, M.; ORR, R. D.

Near-Field Gain of a Horn and an Open-Ended Waveguide; Comparison Between Theory and Experiment

IEEE Trans. Antennas Propagat., Vol. AP-35, No. 1, pp. 33-40

January 1987

LARSEN, E. B.

Calibration and Meaning of Antenna Factor and Gain for EMI Antennas

Interference Technology Engineers' Master (ITEM 1986), pp. 113-335

1986

HILL, D. A.

Far-Field Transient Response of an Antenna from Near-Field Data

NBSIR 86-3063

December 1986

HUL, D. A.; KOEPKE, G. H.

A Near-Field Array of Yagi-Uda Antennas for Electromagnetic Susceptibility Testing

IEEE Trans. Electromagn. Compat., Vol. EMC-28, No. 4, pp. 273-276

November 1986

BAIRD, R. C.; DAYWITT, W. C.; NEWELL, A. C.; PERERA, S.; REPJAR, A. G.; WAIT, D. F.; ESTIN, A. J.

Calibration Requirements for EHF Satellite Communication Systems

NBSIR 86-3058

October 1986

FITZGERRELL, R. G.

Standard Linear Antennas, $30 \mathrm{GHz}$ to $1000 \mathrm{MHz}$

Proc., 5th Intl. Conf. on Electromagn. Compat., 29 September-2 October 1986, University of York, England, pp. 147-153

October 1986

KANDA, M.; ORR, R. D.

Near-Field Gain of a Horn and an Open-Ended Waveguide; Comparison Between Theory and

Experiment

Proc., 5th Intl. Conf. on Electromagn. Compat., 29 September-2 October 1986, University of York, England, pp. 137-145

October 1986 
MUTH, L. A.

Gradient Displacement Errors in Antenna Near-Field Measurements and Their Effects on the Far Field NBS TN 1306

October 1986

HILL, D. A.; FRANCIS, M. H.

Out-of-Band Response of Antenna Arrays

NBSIR 86-3047

June 1986

KANDA, M.; ORR, R. D.

Near-Field Gain of a Horn and an Open-Ended Waveguide; Comparison Between Theory and Experiment

Proc., 1986 Intl. IEEE Antenna Propagat Soc. Symp., 8-13 June 1986, Philadelphia, PA, pp. 91-94

June 1986

NEWELL, A. C.; STUBENRAUCH, C. F.

The Effect of Random Errors in Planar Near-Field Measurements

Proc., 1986 Intl. IEEE Antenna Propagat. Soc. Symp., 8-13 June 1986, Philadelphia, PA, pp. $195-198$

June 1986

FITZGERRELL, R. G.

Linear Gain - Standard Antennas Below $1000 \mathrm{MHz}$

NBS TN 1098

May 1986

JESCH, R. L.

Measured Vehicular Antenna Performance

National Institute of Justice, NIJ Report-201-85

May 1986

NEWELL, A. C.; STUBENRAUCH, C. F.; BAIRD, R. C.

Calibration of Microwave Antenna Gain Standards

Proc. IEEE, Spec. Issue on Radio Meas. Methods and Standards, Vol. 74, Vol. 1, pp. 129-132 January 1986

MUTH, L. A.

Interelement Interactions in Phased Arrays: Theory, Methods of Data Analysis, and Theoretical Simulations

NBS TN 1091

December 1985

YAGHJIAN, A. D.; WITTMANN, R. C.

The Receiving Antenna as a Linear Differential Operator: Application to Spherical Near-Field Scanning IEEE Trans. Antennas Propagat., Vol. AP-33, No. 11, pp. 1175-1185

November 1985

NEWELL, A. C.; REPJAR, A. G.

Development of Near-Field Test Procedures for Communication Satellite Antennas, Phase I, Part 1 NBSIR 85-3031

September 1985 
A Theory of Mutual Impedances and Multiple Reflections in an N-Element Array Environment

Proc., 1985 Intl. Symp. Antennas and Electromagn. Theory, 26-28 August 1985, Beijing, China, pp. 440-443

August 1985

HILL, D. A.; KOEPKE, G. H.

An Array of Dipoles for Plane Wave Synthesis

Proc., 1985 Intl. Symp. Antennas and Electromagn. Theory, 26-28 August 1985, Beijing, China, pp. $422-427$

August 1985

MUTH, L. A.

A Theory of Mutual Impedances and Multiple Reflections in an N-Element Array Environment Proc., 1985 Intl. Symp. Antennas Propagat., 20-22 August 1985, Kyoto, Japan, pp. 719-722 August 1985

HILL, D. A.; KOEPKE, G. H.

A Near-Field Array of Yagi-Uda Antennas for Electromagnetic Susceptibility Testing NBS TN 1082 July 1985

NEWELL, A. C.; FRANCIS, M. H.; KREMER, D. P.; GRIMM, K. R.

Results of Planar Near-field Testing with Ultralow Sidelobe Antennas

Dig., Intl. IEEE Antenna Propagat. Soc. Symp. on Antennas and Propagat., 17-21 June 1985,

University of British Columbia, Vancouver, Canada, pp. 693-698 June 1985

JESCH, R. L.

Measured Vehicular Antenna Performance

IEEE Trans. Veh. Technol., Vol. VT-34, No. 2, pp. 97-107

May 1985

HILL, D. A.

Out-of-Band Response of Reflector Antennas

NBSIR 85-3021

April 1985

WYSS, J. C.; SHEERAN, S.

A Passive, Optical Modulator and Link for Antennas

IEEE J. Lightwave Technol., Vol. LT-3, No. 2, pp. 316-321

April 1985

CRUZ, J. E.; LARSEN, E. B.

Screenroom Measurements of Antenna Factors

Proc., 1985 Instrum. and Meas. Technol. Conf., 20-22 March 1985, Tampa, FL, p. 208 March 1985

LARSEN, E. B.; CRUZ, J. E.

$\mathrm{E}$ and H Fields in Transmission Lines and Coils for Susceptibility Testing, Probe Calibration, and RF

Exposure Chambers

Proc., 1985 Instrum. and Meas. Technol. Conf., 20-22 March 1985, Tampa, FL, p. 199 March 1985 
MUTH, L. A.

A Theory of Mutual Impedances and Multiple Reflections in an N-Element Array Environment NBS TN 1078

February 1985

KANDA, M.

Transients in a Resistively Loaded Loop Antenna

Proc., 1984 Intl. Symp. on Electromagn. Compat., 16-18 October 1984, Tokyo, Japan, Vol. 1, pp. 286-290

October 1984

NEWELL, A. C.; FRANCIS, M. H.; KREMER, D. P.

The Determination of Near-Field Correction Parameters for Circularly Polarized Probes Proc., 1984 Antenna Meas. Tech. Assoc. Conf., 2-4 October 1984, Atlanta, GA, pp. 3A3-1 through 3A3-29

October 1984

STUBENRAUCH, C. F.; FRANCIS, M. H.

Comparison of Measured and Calculated Mutual Coupling in the Near Field Between Microwave Antennas

Proc., 1984 Antenna Applications Symp., 19-21 September 1984, University of Illinois, Urbana-Champaign, IL, pp. 1-21

September 1984

WYSS, J. C.; ANSON, W. J.; ORR, R. D.

Building Penetration Project

NBSIR 84-3009

September 1984

DAYWITT, W. C.

A Preliminary Investigation into Using the Sun as a Source for G/T Measurements NBSIR 84-3015

August 1984

KANDA, M.

An Electromagnetic Near-Field Sensor for Simultaneous Electric and Magnetic-Field Measurements

IEEE Trans. Electromagn. Compat., Vol. EMC-26, No. 3, pp. 102-110

August 1984

STUBENRAUCH, C. F.; FRANCIS, M. H.

Comparison of Measured and Calculated Mutual Coupling in the Near Field Between Microwave Antennas

NBSIR 84-3010

June 1984

WITTMANN, R. C.

Probe Correction in Spherical Near-field Scanning Viewed as an Ideal Probe Measuring an Effective Field

Dig., 1984 Intl. Symp. Antenna Propagat., 25-29 June 1984, Boston, MA, pp. 674-677 June 1984 
FITZGERRELL, R. G.

A Source of $\mathrm{E}$ and $\mathrm{H}$ Fields for Antenna-Factor Calibration

IEEE Trans. Electromagn. Compat., Vol. EMC-26, No. 2, pp. 58-65

May 1984

DAYWITT, W. C.

An Error Analysis for the Use of Presently Available Lunar Radio Flux Data in Broadbeam

Antenna-System Measurements

NBS TN 1973

February 1984

HILL, D. A.

Theory of Near-Field Phased Arrays for Electromagnetic Susceptibility Testing NBS TN 1072

February 1984

MA, M. T.

Arrays of Discrete Elements

Antenna Engineering Handbook, Chapter 3, R. C. Johnson and H. Jasik, eds., McGraw-Hill Book Co.

January 1984

GREENLEE, D. H.; KANDA, M.; CHANG, D. C.

The Characteristics of Iris-Fed Millimeter Wave Rectangular Microstrip Patch Antennas NBS TN 1063

October 1983

BENSEMA, W. D.

Handbook for Broadband Isotropic Antenna System Volume 1 - Operations Manual NBSIR 83-1693

July 1983

YAGHJIAN, A. D.

Approximate Formulas for the Far Field and Gain of Open-Ended Rectangular Waveguide NBSIR 83-1689

May 1983

REPJAR, A. G.; NEWELL, A. C.; BAIRD, R. C.

Antenna Gain Measurements by an Extended Version of the NBS Extrapolation Method

IEEE Trans. Instrum. Meas., Vol. IM-32, No. 1, pp. 88-91

March 1983

WAIT, D. F.

Precision Measurement of Antenna System Noise Using Radio Stars

IEEE Trans. Instrum. Meas., Vol. IM-32, No. 1, pp. 110-116

March 1983

BAIRD, R. C.; ESTIN, A. J.

The Orbiting Standards Package: A Recalibratable Satellite Instrument Assembly for Measuring Large Earth Station Antennas

Proc., 1982 Antenna Meas. Tech. Assoc., 5-7 October 1982, New Mexico State University, Las

Cruces, NM, pp. 5-1 through 5-9

October 1982 
FITZGERRELL, R. G.

A Partial Loop Source of E and H Fields for Antenna Factor Calibration (A Loop Cell)

Proc., 1982 Antenna Meas. Tech. Assoc., 5-7 October 1982, New Mexico State University, Las Cruces, NM, pp. 15-1 through 15-22

October 1982

FRANCIS, M. H.; YAGHJIAN, A. D.

Computations of Antenna Side-Lobe Coupling in the Near Field Using Approximate Far-Field Data NBSIR 82-1674

August 1982

REPJAR, A. G.; NEWELL, A. C.; BAIRD, R. C.

Antenna Gain Measurements by an Extended Version of the NBS Extrapolation Method Dig., 1982 Conf. on Prec. Electromagn. Meas., 28 June-1 July 1982, Boulder, CO, pp. F-7 through F-9

June 1982

STUBENRAUCH, C. F.; SPIESS, W.; GALLIANO, P. G.; BABJI, T.

International Intercomparison of Electric Field Strength at $100 \mathrm{MHz}$

Dig., 1982 Conf. on Prec. Electromagn. Meas., 28 June-1 July 1982, Boulder, CO, pp. P-3 through P-4

June 1982

WAIT, D. F.

Precision Measurement of Antenna System Noise Using Radio Stars

Dig., 1982 Conf. on Prec. Electromagn. Meas., 28 June-1 July 1982, Boulder, CO, p. F-17

June 1982

WYSS, J. C.; KANDA, M.; MELQUIST, D. G.; ONDREJKA, A. R.

Optical Modulator and Link for Broadband Antennas

Dig., 1982 Conf. on Prec. Electromagn. Meas., 28 June-1 July 1982, Boulder, CO, pp. P-16 through $\mathrm{P}-17$

June 1982

KANDA, M.; CHANG, D. C.; GREENLEE, D. H.

The Characteristics of Iris-Fed Millimeter Wave Rectangular Microstrip Patch Antennas

Proc., 1982 IEEE Antenna Propagat. Soc. Symp., 24-28 May 1982, University of New Mexico, Albuquerque, NM, pp. 292-295

May 1982

KANDA, M.

The Effects of Resistive Loading on TEM Horns

IEEE Trans. Electromagn. Compat., Vol. EMC-24, No. 2, pp. 245-255

May 1982

REPJAR, A. G.; KREMER, D. P.

Accurate Evaluation of a Millimeter Wave Compact Range Using Planar Near-Field Scanning

IEEE Trans. Antennas Propagat., Vol. AP-30, No. 3, pp. 419-425

May 1982

WLSON, P. F.; CHANG, D. C.; MA, M. T.

Input Impedance of a Probe Antenna Exciting a TEM Cell

NBS TN 1054

April 1982 
YAGHJAN, A. D.

A Delta-Distribution Derivation of the Electric Field in the Source Region

J. Electromagn. Soc., Vol. 2, No. 2, pp. 161-167

April-June 1982

ESTIN, A. J.; STUBENRAUCH, C. F.; REPJAR, A. G.; NEWELL, A. C.

Optimized Wavelength-Sized Scalar Horns as Antenna Radiation Standards

IEEE Trans. Instrum. Meas., Vol. IM-31, No. 1, pp. 53-56

March 1982

YAGHJIAN, A. D.

Efficient Computation of Antenna Coupling and Fields Within the Near-Field Region

IEEE Trans. Antennas Propagat., Vol. AP-30, No. 1, pp. 113-128

January 1982

MA, M. T.

Synthesis of Broadband Antenna Arrays as Possible Over-the-Horizon Radars Research Topics in EM Wave Theory

Chapter 9, John Wiley Interscience, pp. 188-209 1981

TREADO, M. J.; TAGGART, H. E.; NELSON, R. E.; WORKMAN, J. L.

Fixed and Base Station Antennas

National Institute of Justice (NIJ) Standard-0204.01 (supersedes National Institute for Law

Enforcement and Criminal Justice (NILECJ) STD-0204.00, dated November 1977)

December 1981

BAIRD, R. C.

Microwave Antenna Measurement Services at the National Bureau of Standards

Dig., Antenna Meas. Tech. Assoc. Mtg.

October 1981

BENSEMA, W. D.

Broadband Orthogonal Array Antenna System: Microprocessor Control and Computation

Record, "EMC '81," 1981 IEEE Intl. Symp. on Electromagn. Compat., 18-20 August 1981,

Boulder, $\mathrm{CO}$

August 1981

REEVE, G. R.

Synthesized Isotropic Pattern Antennas for EM Field Measurements

Record, "EMC '81," 1981 IEEE Intl. Symp. on Electromagn. Compat., 18-20 August 1981,

Boulder, $\mathrm{CO}$

August 1981

KERNS, D. M.

Plane-Wave Scattering Matrix Theory of Antennas and Antenna-Antenna Interactions

NBS MN 162

June 1981

LEWIS, R. L.

Spherical-Wave Source-Scattering-Matrix Analysis of the Mutual Coupling Between Two Antennas

Dig., 1981 IEEE Antenna Propagat. Soc. Intl. Symp., pp. 261-264

June 1981 
LEWIS, R. L.

Spherical Scanning Data Processing: An Algorithm for Halving the Data Processing Effort when the Radiation into the Back Hemisphere is Negligible

Dig., 1981 IEEE Antenna Propagat. Soc. Intl. Symp., pp. 247-249

June 1981

REEVE, G. R.; WAINWRIGHT, A. E.

A Frequency Tracking, Tuned, Receiving Monopole

Dig., 1981 IEEE Antenna Propagat. Soc. Intl. Symp.

June 1981

STUBENRAUCH, C. F.; YAGHJIAN, A. D.

Determination of Mutual Coupling Between Co-Sited Microwave Antennas and Calculation of

Near-Zone Electric Field

NBSIR 81-1630

June 1981

YAGHJIAN, A. D.

A Delta-Distribution Derivation of the Electric Field in the Source Region

Dig., 1981 IEEE Antenna Propagat. Soc. Intl. Symp., pp. 334-337

June 1981

LEWIS, R. L.

Efficient Computation of the Far-Field Radiated by an Arbitrary Rectangular-Aperture Distribution

NBSIR 81-1643

April 1981

YAGHJIAN, A. D.

An Approximate Expression for the Principal Beamwidth of Directive Antennas in Terms of Aperture Fields

NBSIR 81-1644

March 1981

REPJAR, A. G.; KREMER, D. P.

Results of Planar Near-Field Measurements on a Compact Range at 18 and $54 \mathrm{GHz}$

Dig., IEEE Antenna Propagat. Soc. Symp.

1980

HILL, D. A.; WAIT, J. R.

Electromagnetic Characteristics of a Coaxial Cable with Periodic Slots

IEEE Trans. Electromagn. Compat., Vol. EMC-22, No. 4, pp. 303-307

November 1980

WAIT, J. R.; HILL, D. A.

Fields of a Horizontal Loop of Arbitrary Shape Buried in a Two-Layer Earth

Radio Sci., Vol. 15, No. 5, pp. 903-912

September-October 1980

KANDA, M.

The Characteristics of a Linear Antenna with Tapered Resistive and Capacitive Loading

Proc., 1980 IEEE Antenna Propagat. Soc. Intl. Symp., 2-6 June 1980, Quebec, Canada, Vol. 2,

AP. $18-2$, pp. 696-699

June 1980 
Antennas (cont.)

KANDA, M.

Transients in Resistively Loaded Antennas and Their Comparison with Conical Antennas and TEM Horns

IEEE Trans. Antennas Propagat., Vol. AP-28, No. 1, pp. 132-136

January 1980

KANDA, M.

Analytical and Numerical Techniques for Analyzing an Electrically Short Dipole with a Nonlinear Load

IEEE Trans. Antennas Propagat., Vol. AP-28, No. 1, pp. 71-78 January 1980

STUBENRAUCH, C. F.

Some Recent Near-Field Antenna Measurements

Proc., 1979 Antenna Application Symp., September, 1979

September 1979

KANDA, M.

The Effects of Resistive Loading on TEM Horns

NBSIR 79-1601

August 1979

MA, M. T.

A Theoretical Study of Unbalanced Ground Effects on Receiving Dipoles

NBSIR 79-1605

May 1979

KANDA, M.

The Time Domain Characteristics of a Traveling Wave Linear Antenna with Linear and Non-Linear Loads

NBSIR 78-892 (Restricted)

February 1979

WACKER, P. F.

Plane-Radial Scanning Techniques With Probe Correction; Natural Orthogonalities with Respect to Summation on Planar Measurement Lattices

Dig., IEEE Antenna Propagat. Soc. Symp., pp. 561-564

February 1979

WACKER, P. F.

Qualitative Survey of Near-Field Analysis and Measurement

NBSIR 79-1602

January 1979

KANDA, M.

Analytical and Numerical Techniques for Analyzing an Electrically Short Dipole with a Nonlinear Load

NBSIR 78-898

November 1978

LAWTON, R. A.; ONDREJKA, A. R.

Antennas and the Associated Time Domain Range for the Measurement of Impulsive Fields NBS TN 1008

November 1978 
TAGGART, H. E.; SHAFER, J. F.

Testing of Electronic Industries Association Land-Mobile Communication Antenna Gain Standards at the National Bureau of Standards

IEEE Trans. Veh. Technol. Vol. VT-27, pp. 259-264

November 1978

DAYWITT, W. C.

An Error Analysis for the Measurement of Satellite EIRP using a Calibrated Radio Star

IEEE Trans. Instrum. Meas., Vol. IM-27, No. 3, pp. 253-258

September 1978

DOUGHERTY, H. T.; ESTIN, A. J.; MORGAN, W. L.; WOODRUFF, J. J.

The Orbiting Standards Platform

Proc., 1978 Antenna Applications Symp., 20-22 September 1978, Urbana-Champaign, IL, pp. 1-9

September 1978

ESTIN, A. J.; BAIRD, R. C.

Feasibility Study of Orbiting Standards Platform

NBSIR 78-886

June 1978

KANDA, M.

A Relatively Short Cylindrical Broadband Antenna with Tapered Resistive Loading for Picosecond Pulse Measurements

IEEE Trans. Antennas Propagat., Vol. AP-26, No. 3, pp. 439-447

May 1978

KANDA, M.

A Relatively Short Cylindrical Broadband Antenna with Tapered Resistive Loading for Picosecond Pulse Measurements

Dig., IEEE Antennas Propagat. Soc., pp. 230-233

May 1978

DAYWITT, W. C.

Atmospheric Propagation Equations Used in the NBS Earth Terminal Measurement System NBSIR 78-883

April 1978

WAIT, D. F.

Earth Terminal Measurement System Operations Manual

NBSIR 78-879

April 1978

DAY, G. W.; STUBENRAUCH, C. F.

Laser Far-Field Beam-Profile Measurements by the Focal Plane Technique NBS TN 1001

March 1978

KANDA, M.

Transients in Resistively Loaded Antennas and Their Comparison with Conical Antennas and TEM Horns

NBSIR 78-876

March 1978 
WACKER, P. F.

Symmetry Analysis Applied to Wave Theory

Proc., Intl. IEEE Antenna Propagat. Soc. Symp., pp. 169-172

1977

WACKER, P. F.

Symmetry Analysis Applied to Scattering, Inverse Scattering, and Antenna Patterns: Measurements,

Moment Method, and Characteristic Modes

Proc., 1977 Intl. IEEE Antenna Propagat. Soc. Symp., pp. 177-180

1977

DAYWITT, W. C.

Error Equations Used in the NBS Earth-Terminal Measurement System

NBSIR 78-869

December 1977

YAGHJLAN, A. D.

A Direct Approach to the Derivation of Electric Dyadic Green's Functions

NBS TN 1000

December 1977

DAYWITT, W. C.

A Precision Earth-Terminal System for Accurate C/KT, G/T, and EIRP Measurements with a Calibrated Radio Star

Proc., 1977 Intl. Union of Radio Sci. (Union Radio Scientifique Internationale) Symp. on Meas. in Telecommunications, 3-7 October 1977, Lannion, France, pp. 1-4

October 1977

MA, M. T.; FITZGERRELL, R. G.

Design of a Van-Top Low-Profile HF Antenna

OTR-77-131

October 1977

YAGHJIAN, A. D.

Near-Field Antenna Measurements on a Cylindrical Surface: A Source Scattering-Matrix Formulation NBS TN 696

September 1977

KANDA, $M$.

A Relatively Short Cylindrical Broadband Antenna with Tapered Resistive Loading for Picosecond Pulse Measurements

NBSIR 77-861

August 1977

\section{LEWIS, R. L.}

The Use of Three Term Recursion Relations for Numerical Computations as Applied to Near-Field Spherical Scanning

Proc., Intl. Union of Radio Sci. (Union Radio Scientifique Internationale) Intl. Symp.,

20-24 June 1977, pp. 224-226

June 1977 
WACKER, P. F.

Recent Results with Spherical Near-Field Antenna Scanning at the National Bureau of Standards Proc., 1977 European Space Agency Symp., 6-8 June 1977, Noordwijk, Netherlands, pp. 159-164

June 1977

WACKER, P. F.; NEWELL, A. C.

Advantages and Disadvantages of Planar, Circular Cylindrical and Spherical Scanning and Description of the NBS Antenna Scanning Facilities

Proc., 1977 European Space Agency Symp., 6-8 June 1977, Noordwijk, Netherlands, pp. $115-121$

June 1977

WAIT, D. F.

Satellite Earth Terminal G/T Measurements

Microwave J., Vol. 20, No. 4, pp. 49, 51, 58

April 1977

YAGHJIAN, A. D.

A Direct Approach to the Derivation of Electric Dyadic Green's Functions

Dig., Antenna Propagat. Soc. Symp., pp. 76-156

October 1976

DAYWITT, W. C.

Error Equations Used in the NBS Precision G/T Measurement System

NBSIR 76-842

September 1976

KANDA, $M$.

Study of Error Analysis for Absolute Flux Density Measurements of Cassiopeia

IEEE Trans. Instrum. Meas., Vol. IM-25, No. 3, pp. 173-182

September 1976

LARSEN, E. B.; ANDREWS, J. R.

Using Fiber Optics in a Broadband, Sensitive, Isotropic Antenna - $15 \mathrm{kHz}$ to $150 \mathrm{MHz}$

Proc., IEEE Intl. Symp. on Electromagn. Compat., 13-15 July 1976, Washington, DC, pp. 385-389

July 1976

CRAWFORD, M. L.

Calibration of Broadbeam Antennas Using Planar Near-Field Measurements

Proc., 1976 Conf. on Prec. Electromagn. Meas., 28 June-1 July 1976, Boulder, CO, pp. 53-56 June 1976

NEWELL, A. C.; REPJAR, A. G.

Results of Spherical Near-Field Measurements on Narrow-Beam Antennas

Proc., 1976 Conf. on Prec. Electromagn. Meas., 28 June-1 July 1976, Boulder, CO, pp. 382-385 June 1976

WACKER, P. F.

Non-Planar Near-Field Measurements: Spherical Scanning

NBSIR 75-809

1975 
YAGHJIAN, A. D.

Upper-Bound Errors in Far-Field Antenna Parameters Determined from Planar Near-Field Measurements.

Part 1: Analysis

NBS TN 667

October 1975

DAYWITT, W. C.; KANDA, M.

G/T Measurement Errors with Radio Stars

Proc., 1975 IEEE Intl. Antenna and Propagat. Symp., 2-4 June 1975, Urbana-Champaign, IL, Session 20,pp. 460-463

June 1975

NEWELL, A. C.

Improved Polarization Measurements Using a Modified Three Antenna Technique

Proc., 1975 IEEE Intl. Antenna and Propagat. Symp., 2-4 June 1975, Urbana-Champaign, IL, Session 15, pp. 337-340

June 1975

NEWELL, A. C.; YAGHJAN, A. D.

Study of Errors in Planar Near-Field Measurements

Proc., 1975 IEEE Intl. Antenna and Propagat. Symp., 2-4 June 1975, Urbana-Champaign, IL, Session 20, pp. 470-473

June 1975

KANDA, M.

Accuracy Considerations in the Measurement of the Power Gain of a Large Microwave Antenna

IEEE Trans. Antennas Propagat., Vol. AP-23, No. 3, pp. 407-411

May 1975

KERNS, D. M.

Plane-Wave Scattering Matrix Theory of Antennas and Antenna-Antenna Interactions: Formulation and Applications

NBSIR 75-824

January 1975

MA, M. T.

Theory and Application of Antenna Arrays

John Wiley Interscience

1974

WACKER, P. F.

Near-Field Antenna Measurements Using a Spherical Scan: Efficient Data Reduction with Probe

Correction

Dig., Conf. on Prec. Electromagn. Meas., No. 113, pp. 286-288 London, England 1974

NEWELL, A. C.; CRAWFORD, M. L.

Planar Near-Field Measurements on High Performance Array Antennas

NBSIR 74-380

July 1974 
BUSSEY, H. E.; LARSEN, E. B.

Buried Antenna Performance: Development of Small Resonant Buried Antennas

RADC-TR-74-169M, AD 783274

June 1974

KANDA, M.

Accuracy Considerations in the Measurement of the Power Gain of a Large Microwave Antenna

Proc., 1974 Intl. Antenna Propagat. Soc. Symp., 10-12 June 1974, Atlanta, GA, pp. 43-45 June 1974

NEWELL, A. C.; CRAWFORD, M. L.

Planar Near-Field Measurements on Phased Array Antennas

Proc., 1974 Intl. IEEE Antenna Propagat. Soc. Symp., 10-12 June 1974, Atlanta, GA, p. 423

June 1974

WACKER, P. F.

Antenna Measurements at the National Bureau of Standards Near-Field and Extrapolation Techniques Proc., 1974 Colloquium on Microwave Communication 24-30 June 1974, Budapest, PME-113-114

June 1974

BAIRD, R. C.; KERNS, D. M.

The Accurate Determination of Millimeter-Wave Antenna Characteristics by Deconvolution and Extrapolation Techniques

Proc., 1974 Millimeter-Wave Conf., 26-28 March, 1974, Vol. 2, pp. E 2-1 through E 2-12 March 1974

NEWELL, A. C.; BAIRD, R. C.; WACKER, P. F.

Accurate Measurement of Antenna Gain and Polarization at Reduced Distances by an Extrapolation Technique

IEEE Trans. Antennas Propagat., Vol. AP-21, No. 4, pp. 418-431 July 1973

KERNS, D. M.

Comment on Correction of Errors in Aerial Far-Field Radiation-Pattern Determinations

Electronics Lett., Vol. 7, No. 24, pp. 706

December 1971

NEWELL, A. C.; KERNS, D. M.

Determination of Both Polarization and Power Gain of Antennas by a Generalized 3-Antenna Measurement Method

Electronics Lett., Vol. 7, No. 3, pp. 68-70

January 1971 
GEYER, R. C.; BAKER-JARVIS, J. R.; VANDERAH, T. A.; MANTESE, J. F

Complex Permittivity and Permeability Estimation of Composite Electroceramics

Advances in Dielectric Ceramic Materials, Vol. 88, pp. 115-129

May 1998

GEYER, R. G.; JONES, C. A.; KRUPKA, J.

Complex Permeability Measurements of Ferrite Ceramics Used in Wireless Communications

Advances in Dielectric Ceramic Materials, Vol. 88, pp. 93-113

May 1998

GEYER, R. G.; JONES, C. A.; KRUPKA, J.

Microwave Characterization of Dielectric Ceramics for Wireless Communiucations

Advances in Dielectric Ceramic Materials, Vol. 88, pp. 75-91

May 1998

KRUPKA, J.; WEIL, C. M.

Recent Advances in Metrology for the Electromagnetic Characterization of Bulk Materials at Microwave Frequencies

Proc., MIXON XII, Intl. Microwave Conf., 20-22 May 1998, Krakow, Poland, pp. 243-253 May 1998

JANEZIC, M. D.; WILLIAMS, D. F.

Permittivity Characterization from Transmission-Line Measurement

Dig., IEEE 1997 MTT Intl. Symp., 8-12 June 1997, Denver, CO, pp. 1343-1345

June 1997

KRUPKA, J.; DERZAKOWSKI, K.; ABRAMOWICZ, A.; TOBAR, M.; GEYER, R. G.

Complex Permittivity Measurements of Extremely Low Loss Dielectric Materials Using Whispering

Gallery Modes

Dig., IEEE 1997 MTT Intl. Symp., 8-12 June 1997, Denver, CO, pp. 1347-1350 June 1997

LEWIS, R. L.

Relative Permittivity Measurement of Rectangular Copper-Laminated Substrates Using the Full-Sheet

Resonance Technique

NIST IR 5062

April 1997

WEIL, C. M.; JANEZIC, M. D.; VANZURA, E. J.

Intercomparison of Permeability and Permittivity Measurements Using the Transmission/Reflection Method in 7 and $14 \mathrm{~mm}$ Coaxial Air Lines

NIST TN 1386

March 1997

LEWIS, R. L.

Relative Permittivity Measurement of Square Copper-Laminated Substrates Using the Full Sheet

Resonance Technique

NISTIR 5053

January 1997

BAKER-JARVIS, J. R.; RIDDLE, B. F.

Dielectric Measurements Using a Reentrant Cavity: Mode-Matching Analysis

NIST TN 1384

November 1996 
KRUPKA, J.; GEYER, R. G.; BAKER-JARVIS, J. R.; CEREMUGA, J.

Measurements of the Complex Permittivity of Microwave Circuit Board Substrates Using Split Dielectric Resonator and Reentrant Cavity Techniques

Proc., 7th Intl. Conf. Dielectric Materials Meas. Appli., 23-26 September 1996, Bath, England Pub. No. 430, pp. 21-24

September 1996

GEYER, R. G.; KRUPKA, J.; SENGUPTA, L.; SENGUPTA, S.

Microwave Properties of Composite Ceramic Phase Shifter Material

Proc., IEEE Intl. Symp. for Apps. of Ferroelectrics, 18-21 August 1996, East Brunswick, NJ, pp. 851-854

August 1996

JONES, C. A.; KANTOR, Y.; GROSVENOR, J. H.

Permittivity Measurements of Low-Loss Dielectric Materials at $60 \mathrm{GHz}$

Proc., Intl. Conf. Millimeter and Submillimeter Waves Appli., 5-7 August 1996, Denver, CO, pp. 263-273

August 1996

KRUPKA, J.; BLONDY, P.; CROS, D.; GUILLON, P.; GEYER, R. G.

Whispering-Gallery Modes and Permeability Tensor Measurements in Magnetized Ferrite Resonators IEEE Microwave Theory Tech., Vol. 44, No. 7, pp. 1097-1102

July 1996

GEYER, R. G.; KRUPKA, J.

Microwave Behavior of Ferrites: Theory and Experiment

Dig., 1996 Conf. on Prec. Electromag. Meas., 17-20 June 1996; Braunschweig, Germany;

pp. 206-207

June 1996

KRUPKA, J.; PIETRUSZKO, S.; GEYER, R. G.; BAKER-JARVIS, J. B.; DERZAKOWSKI, K.

Semiconductors Resistivity Measurements Using Split-Dielectric Resonator Technique

Proc., MIKON XI, Intl. Microwave Conference, 27-30 May 1996, Warsaw, Poland, pp. 361-364

May 1996

KRUPKA, J.; GEYER, R. G.

Complex Permeability of Demagnetized Microwave Ferrites Near and Above Gyromagnetic Resonance

IEEE Trans. Magn. Vol. 32, No. 3, pp. 1924-1933

May 1996

GEYER, R. G.; KRUPKA, J.

Complex Permeability Measurements of Microwave Ferrites

Proc., Materials Research Soc., 7-12 April 1996, San Francisco, CA, pp. 257-262 April 1996

BAKER-JARVIS, J. R.; GROSVENOR, J. H.

Dielectric and Magnetic Measurements at Elevated Temperatures in the Frequency Band $50 \mathrm{MHz}$ to $2 \mathrm{GHz}$ NISTIR 5045

March 1996 
BAKER-JARVIS, J. R.; JANEZIC, M. D.

Analysis of a Two-Port Flanged Coaxial Holder for Shielding Effectiveness and Dielectric Measurements of Thin Films and Thin Materials

IEEE Trans. Electromag. Compat., Vol. 38, No. 1, pp. 67-70

February 1996

MANTESE, J. F.; MICHELI, A. L.; DUNGAN, D. F.; GEYER, R. G.; BAKER-JARVIS, J. R.;

GROSVENOR, J. H.

Applicability of Effective Medium Theory to Ferroelectric/Ferrimagnetic Composites with Composition and Frequency-Dependent Complex Permittivities and Permabilities

J. Appl. Phys., Vol. 79, No. 3, pp. 1655-1660

February 1996

BAKER-JARVIS, J. R.; JONES, C. A.; RIDDLE, B. F.; JANEZIC, M. D.; GEYER, R. G.; GROSVENOR, J. H.; WEL, C.M.

Dielectric and Magnetic Measurements: A Survey of Nondestructive, Quasi-Nondestructive, and ProcessControl Techniques

Research in Nondestructive Evaluation, Vol. 7, pp. 117-136

1995

VANDERAH, T. A.; HUANG, Q.; WONG-NG, W.; CHAKOUMAKOS, B. C.; GOLDFARB, R. B.;

GEYER, R. G.; BAKER-JARVIS, J. R.; ROTH, R. S.; SANTORO, A.

Preparation, Crystal Structure, Dielectric Properties, and Magnetic Behavior of $\mathrm{Ba}_{2} \mathrm{Fe}_{2} \mathrm{Ti}_{4} \mathrm{O}_{13}$ J. Solid State Chem., Vol. 120, pp. 121-127

November 1995

WEח, C. M.

The NIST Metrology Program on Electromagentic Characterization of Materials

Proc., Symp. on Materials and Processes for Wireless Communication, 15-16 Nov. 1995,

Boston, MA, pp. 35-48

November 1995

BAKER-JARVIS, J. R.; JONES, C. A.

Dielectric Measurements on Printed-Wiring and Circuit Boards, Thin Films, and Substrates:

An Overview

Proc., Materials Res. Soc., April 18, 1995, San Francisco, CA, pp. 153-164 April 1995

GEYER, R. G.; KRUPKA, J.

Microwave Dielectric Properties of Anisotropic Materials at Cryogenic Temperatures

IEEE Trans. Instrum. Meas., Vol. 44, No. 2, pp. 329-331 April 1995

CEREMUGA, J.; KRUPKA, J.; GEYER, R. G.; MODELSKI, J. Influence of Film Thickness and Air Gaps in Surface Impedance Measurements of High Temperature Superconductors Using the Dielectroc Resonator Technique

IEIC Trans. Electron. (Japanese), Vol. E78-C, No. 8, pp. 1106-1110

December 1994

VANZURA, E. J.; BAKER-JARVIS, J. R.; GROVSENOR, J. H.; JANEZIC, M. D. Intercomparison of Permittivity Measurements Using the Transmission/Reflection Method in 7-mm Coaxial Transmission Lines

IEEE Trans. Microwave Theory Tech., Vol. 42, No. 11, pp. 2063-2070 November 1994 
BAKER-JARVIS, J. R.; JANEZIC, M. D.; DOMICH, P. D.; GEYER, R. G.

Analysis of an Open-Ended Coaxial Probe with Lift-Off for Nondestructive Testing

IEEE Trans. Instrumen. Meas., Vol. 43, No. 5, pp. 711-718

October 1994

GEYER, R. G.; KRUPKA, J.; KUHN, M.; HINKEN, J.

Dielectric Properties of Single Crystals of $\mathrm{Al}_{2} \mathrm{O}_{3}, \mathrm{LaAlO}_{3}, \mathrm{NdGaO}_{3}, \mathrm{SrTiO}_{3}$, and $\mathrm{MgO}$ at Cryogenic

Temperatures

IEEE Trans. Microwave Theory Tech., Vol. 42, No. 10, pp. 1886-1889

October 1994

GEYER, R. G.; MANTESE, J. F.; BAKER-JARVIS, J. R.

Effective Medium Theory for Ferrite-Loaded Materials

NIST TN1371

October 1994

JONES, C. A.

Substrate and Thin Film Measurements

Proc., 1994 Wireless Workshop, 16-19 October 1994, Phoenix, AZ, pp. 1-13

October 1994

BAKER-JARVIS, J. R.; JANEZIC, M. D.

Distribution of Dielectric Relaxation Times and the Moment Problem

Dig., 1994 Conf. on Prec. Electromagn. Meas., 27 June-1 July, 1994, Boulder, CO, pp.356-357 June 1994

GEYER, R. G.; KRUPKA, J.

Dielectric Properties of Materials at Cryogenic Temperatures and Microwave Frequencies

Dig., 1994 Conf. on Prec. Electromagn. Meas., 27 June-1 July 1994, Boulder, CO, pp. 350-351 June 1994

KRUPKA, J.; GEYER, R. G.; CROS, D.

Use of Higher-Order TE ${ }_{\text {oy } \delta}$ Modes in Dielectric Measurements of Dielectric Rod Resonators Proc., MIKON '94 Microwave Conf., 30 May-2 June 1994, Ksąiż, Poland, pp. 567-572 June 1994

BAKER-JARVIS, J. R.; JANEZIC, M. D.

Open-Ended Coaxial Probes for Nondestructive Testing of Substrates and Circuit Boards

Proc., Mater. Res. Soc., April 4-8, 1994, San Francisco, CA, pp. 215-220

April 1994

BAKER-JARVIS, J. R.

Dielectric and Magnetic Relaxation by Maximum-Entropy Method

Smart Mater. Struct., Vol. 2, pp. 113-123

1993

GROSVENOR, J. H.

NIST Measurement Service for Electromagnetic Characterization of Materials NISTIR 5006

August 1993 
VANZURA, E. J.; GEYER, R. G.; JANEZIC, M. D.

The NIST 60-Millimeter Diameter Cylindrical Cavity Resonator: Performance Evaluation for Permittivity

Measurements

NIST TN 1354

August 1993

BAKER-JARVIS, J. R.; JANEZIC, M. D.; STAFFORD, R. B.

Shielded Open-Circuited Sample Holders for Dielectric and Magnetic Measurements of Liquids and Powders

NISTIR 5001

March 1993

CLARK, A. V.; SCHAPS, S. R.; BAKER-JARVIS, J. R.; GEYER, R. G.

Development of Electromagnetic Probes for Intelligent Processing of Dielectric Materials

NISTIR 4963, pp. 34-37

November 1992

BAKER-JARVIS, J. R.; GEYER, R. G.; DOMICH, P. D.

A Nonlinear Least-Squares Solution with Causality Constraints Applied to Transmission Line

Permittivity and Permeability Determination

IEEE Trans. Instrum. Meas., Vol. 41, No. 5, pp. 646-652

October 1992

BAKER-JARVIS, J. R.

Dielectric and Magnetic Measurement Methods in Transmission Lines: An Overview

Proc., AMTA Workshop on EM Characterization of Materials for Antenna/RCS Applications, 20-25 July 1992, Chicago, IL, pp. 1-23

July 1992

GEYER, R. G.; BAKER-JARVIS, J. R.; JANEZIC, M. D.; DOMICH, P. D.

Spectral Characterization of Ferrites for Use as Magnetic Reference Materials

Dig., 1992 Conf. on Prec. Electromagn. Meas., 9-12 June 1992, Paris, France, pp. 107-108 June 1992

VANZURA, E. J.; WEIL, C. M.; WILLIAMS, D. F.

Complex Permittivity Measurements of Gallium Arsenide Using a High-Precision Resonant Cavity

Dig., 1992 Conf. on Prec. Electromagn. Meas., 9-12 June 1992, Paris, France, pp. 103-104 June 1992

BAKER-JARVIS, J. R.; JANEZIC, M. D.; GROSVENOR, J. H.; GEYER, R. G.

Transmission/Reflection and Short-Circuit Line Methods for Measuring Permittivity and Permeability

NIST TN 1355

May 1992

WEIL, C. M.

The Electromagnetic Properties of Materials Program at NIST

Proc., Mat. Soc. Symp., 27-30 April 1992, San Francisco, CA, Vol. 269, pp. 517-526 April 1992

CLARK, A. V.; SCHAPS, S. R.; BAKER-JARVIS, J. R.; GEYER, R. G.;

CAPOBIANCO, T. E.; AULD, B. A.

Development of Electromagnetic Probes for Intelligent Processing of Dielectric Materials

NISTIR 4693, pp. 54-61

November 1991 
DOMICH, P. D.; BAKER-JARVIS, J. R.; GEYER, R. G.

Optimization Techniques for Permittivity and Permeability Determination

NIST JRES, Vol. 96, No. 5, pp. 565-575

September/October 1991

DOMICH, P. D.; BAKER-JARVIS, J. R.; GEYER, R. G.

Optimization Techniques for Permittiivity and Permeability Determination

NISTIR 4571

June 1991

JANEZIC, M. D.; GROSVENOR, J. H.

Improved Technique for Measuring Permittivity of Thin Dielectrics with a Cylindrical Resonant Cavity

Conf. Record, IEEE Instrum. Meas. Technol. Conf., 14-16 May 1991, Atlanta, GA, pp. 580-584

May 1991

VANZURA, E. J.; ROGERS, J. E.

Evaluation of a Resonant Circuit Model Using Reflected S-Parameter Data

Record, IEEE Instrum. Meas. Tech.C onf., 14-16 May 1991, Atlanta, GA, pp. 150-155

May 1991

WEIL, C. M.; KISSICK, W. A.

The NIST Electromagnetic Properties of Materials Program

Conf. Record, IEEE Instrum. Meas. Tech. Conf., 14-16 May 1991, Atlanta, GA, pp. 626-630 May 1991

HILL, D. A.

Electric and Magnetic Dipole Radiation in a Random Medium

Electromagnetics, Vol. 10, pp. 279-292

1990

BAKER-JARVIS, J. R.; VANZURA, E. J.; KISSICK, W. A.

Improved Technique for Determining Complex Permittivity with the Transmission/Reflection Method

IEEE Trans. Microwave Theory Tech., Vol. 38, No. 8, pp. 1096-1103

August 1990

BAKER-JARVIS, J. R.

Transmission/Reflection and Short-Circuit Line Permittivity Measurement Methods NIST TN 1341

July 1990

BAKER-JARVIS, J. R.; GEYER, R. G.

Improvements in Transmission Line Permittivity and Permeability Measurements

Dig., Conf. on Prec. Electromagn. Meas., 11-14 June 1990, Ottawa, Canada, pp. 232-233

June 1990

GEYER, R. G.; WEIL, C. M.; KISSICK, W. A.

Precision Dielectric Measurements Using a Mode-Filtered Cylindrical Cavity Resonator

Dig., Conf. on Prec. Electromagn. Meas., 11-14 June 1990, Ottawa, Canada, pp. 174-175 June 1990

GEYER, R. G.

Dielectric Characterization and Reference Materials

NIST TN 1338

April 1990 
GEYER, R. G.

Electrodynamics of Materials for Dielectric Measurement Standardization

Record, Instrum. Meas. Tech. (IMTC) Conf., 13-15 February 1990, San Jose, CA, pp. 2-7

February 1990

GEYER, R. G.; JANEZIC, M. D.; KISSICK, W. A.; BAKER-JARVIS, J. R.

Preliminary Microwave Complex Permittivity Measurements of Heavy-Metal Fluoride Glasses Proc., Third DoD Symp. on Electromagnetic Windows, 14-16 November 1989, Redstone Arsenal, Huntsville, AL, pp. 116-141

November 1989

HILL, D. A.

Near-Field Detection of Buried Dielectric Objects

IEEE Trans. Geosci. Remote Sensing, Vol. GE-27, No. 4, pp. 364-368 July 1989

VANZURA, E. J.; KISSICK, W. A.

Advances in NIST Dielectric Measurement Capability Using a Mode-Filtered Cylindrical Cavity

Proc., 1989 IEEE MTT-S Intl. Microwave Symp., 13-15 June 1989, Long Beach, CA,

pp. 901-904

June 1989

JESCH, R. L.

Measurement of Shielding Effectiveness of Different Cable and Shielding Configurations by

Mode-Stirred Techniques

IEEE Trans. Electromagn. Compat., Vol. EMC-30, pp. 222-228

August 1988

GEYER, R. G.

Dielectric Mixing Rules for Background Test Soils

NBSIR 88-3095

June 1988

LAWTON, R. A.; ANDERSON, W. T.

Two Layer Dielectric Microstrip Line Structure: $\mathrm{SiO}_{2}$ on $\mathrm{Si}$ and $\mathrm{GaAs}$ on Si: Modeling and Measurement

IEEE Trans. Microwave Theory Tech., Vol. MTT-36, No. 4, pp. 785-789

April 1988

DAYWITT, W. C.

Complex Permittivity of Beryllium Oxide Between 100 and 300 Kelvins at 9.3 Gigahertz

IEEE Trans. Instrum. Meas., Vol. IM-34, No. 1, pp. 98-99

March 1985

JESCH, R. L.

Dielectric Measurements of Oil Shale as Functions of Temperature and Frequency

IEEE. Trans. Geosci. Remote Sensing, Vol. GE-22, No. 2, pp. 99-105

March 1984

\section{BUSSEY, H. E.}

Dielectric Measurements in a Shielded Open Circuit Coaxial Line

IEEE Trans. Instrum. Meas., Vol. IM-29, No. 2, pp. 120-124

June 1980 
BUSSEY, H. E.

Open Circuited Coaxial Resonator for High Sensitivity Dielectric Measurements, Application to Lunar Soil 70051-20

Proc., 1979 Lunar and Planetary Science Conf. (10th) Natl. Aeronautics and Space Administration, 19-23 March 1979, Lyndon B. Johnson Space Center, Houston, TX March 1979

BUSSEY, H. E.

Dielectric Measurements of Lunar Soil

Proc., 1979 Lunar and Planetary Science Conf. (10th) Natl. Aeronautics and Space Administration, 19-23 March 1979, Lyndon B. Johnson Space Center, Houston, TX March 1979

JESCH, R. L.

Dielectric Measurements of Five Different Soil Textural Types as Functions of Frequency and Moisture Content

NBSIR 78-896

1978

BENNETT, W.S.; TAGGART, H. E.

Characteristics of A CISPR/VDE Far-Field EMI Test Site with Ground Screen

Proc., Third Rotterdam Electromagn. Compat. Symp.

December 1978

JONES, R. N.; BUSSEY, H. E.; LITTLE, W. E.; METZKER, R. F.

Electrical Characteristics of Corn, Wheat, and Soya in the 1-200 MHz Range

NBSIR 78-897

October 1978

ELLERBRUCH, D. A.; LITTLE, W. E.; BOYNE, H. S.; BACHMAN, D. D.

Microwave Characteristics of Snow

Proc., 1977 Western Snow Conf., 18-21 April 1977, Albuquerque, NM, p. 7 April 1977

BUSSEY, H. E.; RICHMOND, J. H.

Scattering by a Lossy Dielectric Circular Cylindrical Multilayer, Numerical Values

IEEE Trans. Antennas Propagat., Vol. 23, No. 5, pp. 723-725

September 1975

BUSSEY, H. E.; MORRIS, D.; ZALTSMAN, E. B.

International Comparison of Complex Permittivity Measurement at $9 \mathrm{GHz}$

IEEE Trans. Instrum. Meas., Vol. IM-23, No. 3, pp. 235-239

September 1974

LAWTON, R. A.

Autocorrelation and Power Measurement with Pyroelectric and Dielectric Bolometers

IEEE Trans. Instrum. Meas., Vol. IM-22, pp. 299-306

December 1973

BUSSEY, H. E.

Wavelength of a Slotted Rectangular Line Containing Two Dielectrics

NBSIR 73-326

July 1973 
Dielectric Measurements (cont.)

BUSSEY, H. E.

Rapport sur la Comparaison Intl des Mesures de Permittivite Complexe a $9 \mathrm{GHz}$ (Intl. Comparison of Complex Permittivity Measurement at $9 \mathrm{GHz}$ )

Proc., of Comite Consultatif, D'Electricite Comite, Bureau Intl. des Poids et Mesures, 12-13 October 1972, Sevres, France, pp. 124-137

October 1972 


\section{ELECTROMAGNETIC INTERFERENCE}

HILL, D. A.

Spherical-Wave Characterization of Interior and Exterior Sources

NIST IR 5072

December 1997

BUTLER, C. M.; HILL, D. A.; NOVOTNY, D. R.; KANDA, M.

EMI/EMC Metrology Challenges for Industry: A Workshop on Measurements, Standards, Calibrations and Accreditation

NISTIR 5068

November 1997

KWALKO, S. F.; KANDA, M.

The Effective Length and Input Impedance of the NIST Standard Diapole IEEE Trans. Electromagn. Compat., Vol. 39, No. 4, pp. 404-408 November 1997

KWALKO, S. F,; KANDA, M.

The Near-Zone Gain of Open-Ended Rectangular Waveguides

IEEE Trans. Electromagn. Compat., Vol. 39, No. 4, pp. 408-413

November 1997

LADBURY, J. M.; KOEPKE, G. H.; CAMELL, D. G.

Improvements in the $\mathrm{CW}$ Evaluation of Mode-Stirred Chambers

Proc., IEEE EMC Symp., 18-22 August 1997, Austin TX, pp. 33-37

August 1997

BAIRD, R. C.; KANDA, M.

Measurement Related Issues, Problems and Requirements Associated with Electromagnetic

Compatibility: Results of a Limited Survey

NIST IR 5049

July 1997

MASTERSON, K. D.; NOVOTNY, D. R.; KOEPKE, G. H.

Electromagnetic Shielding Characteristics of Optical-Fiber Connectors NIST TN 1383

April 1997

HILL, D. A.; KANDA, M.

Measurement Uncertainty of Radiated Emissions

NIST TN 1389

March 1997

HOLLOWAY, C. L.; DELYSER, R. R.; GERMAN, R. F.; MCKENNA, P.; KANDA, M.

Evaluation and Comparison of Electromagnetic Absorbers Used in Anechoic and Semi-Anechoic

Chambers for Emissions and Immunity Testing of Commercial Equipment

IEEE Trans. Electromagn. Compat., Vol. 39, No. 1, pp. 33-47

February 1997

DELYSER, R. R.; HOLLOWAY, C. L.; JOHNK, R. T.; ONDREJKA, A. R.; KANDA, M.

A New Measure of Quality Factor for Low-Frequency Anechoic Chamber Based on Absorber Reflection Coefficients

IEEE Trans. Electromagn. Compat., Vol. 38, No. 4, pp. 576-584

November 1996 
HILL, D. A.

A Reflection Coefficient Derivation for the Q of a Reverberation Chamber

IEEE Trans. Electromagn. Comp., Vol. 38, No. 4, pp. 591-592

November 1996

KODALI, V. P.; KANDA, M.

EMC/EMI Selected Readings

IEEE Press, Piscataway, NJ

November 1996

JOHNK, R. T.; RANDA, J.

Low Frequency Representation of Radio-Frequency Absorbers

Proc., IEEE Intl. Symp. on Electromagn. Compat., 19-23 August 1996, Santa Clara, CA, pp. $174-179$

August 1996

TOFANI, S.; OSSOLA, P.; D'AMORE, G.; ANGLESIO, L.; KANDA, M.; NOVOTNY, D. R.

A Three-Loop Antenna System for Performing Near Field Measurements of Electric and Magnetic Fields from Video Display Terminals (VDTs)

IEEE Trans. Electromagn. Compat., Vol. 38, No. 3, pp. 341-347

August 1996

LADBURY, J. M.; JOHNK, R. T.; ONDREJKA, A. R.

Rapid Evaluation of Mode-Stirred Chambers Using Impulsive Waveforms

NIST TN 1381

June 1996

LADBURY, J. M.; JOHNK, R. T.; ONDREJKA, A. R.

Rapid Evaluation of Mode-Stirred Chambers Using Impulsive Waveforms NIST TN 1381

June 1996

CAMELL, D. G.; KOEPKE, G. H.; SMITH, R. B.; RAKOSKI, B.

A Standard Source Method for Reducing Antenna Factor Errors in Shielded Room Measurements NIST TN 1382

March 1996

HILL, D.A.

Electromagnetic Shielding Characterization of Gaskets

NIST IR 5032

February 1996

CRAWFORD, M. L.; RIDDLE, B. F.; CAMELL, D. G.

TEM/Reverberating Chamber Electromagnetic Radiation Test Facility at Rome Laboratory NISTIR 5002

January 1996

CRAWFORD, M. L.; LOUGHRY, T. A.; HATFIELD, M. O.; FREYER, G. J.

Band-Limited White Gaussian Noise Excitation for Reverberation Chambers and Applications to

Radiated Susceptibility Testing

NIST TN 1375

January 1996 
CRAWFORD, M. L.

Alternative EMC Compliance Test Facilities

Chapter 18 in Electromagnetic Compatibility Handbook, Academic Press, San Diego, CA 1995

HILL, D. A.

Electric Dipole Excitation of a Long Conductor in a Lossy Medium

Electromagnetics, Vol. 15, pp. 301-319

1995

KANDA, M.

Methodology for Electromagnetic Interference Measurements

Chapter 15 in Electromagnetic Compatibility Handbook, Academic Press, San Diego, CA 1995

KANDA, M.

Standard Antennas for Electromagnetic Field Measurements

Chapter 16 in Electromagnetic Compatibility Handbook, Academic Press, San Diego, CA 1995

CAMELL, D. G.; MA, M. T.

Data Evaluation of a Linear System by a Second Order Transfer Function

Proc., IEEE EMC Symp., 14-18 August 1995, Atlanta, GA, pp. 511-515

August 1995

HILL, D. A.; CAMELL, D. G.; CAVCEY, K. H.; KOEPKE, G. H.

Radiated Emissions and Immunity of Microstrip Transmission Lines: Theory and Measurements TN 1377

July 1995

RANDA, J.
A Low-Frequency Model for Radio-Frequency Absorber
NIST JRES, Vol. 100, No. 3, pp. 257-267
May-June 1995

HILL, D. A.

Spatial Correlation Function for Fields in a Reverberation Chamber

IEEE Trans. Electromagn. Compat. (Correspondence), Vol. 37, No. 1, p. 1 February 1995

RANDA, J.; GILLILAND, D.; GJERTSON, W.; LAUBER, W.; McINERNEY, M.

Catalogue of Electromagnetic Environment Measurements, 30 to $300 \mathrm{~Hz}$

IEEE Trans. Electromagn. Compat., Vol. 37, No. 1, pp. 26-33

February 1995

HILL, D. A.

Gradiometer Antennas for Detection of Long Subsurface Conductors

J. Electromagn. Waves Appl., Vol. 8, No. 2, pp. 237-248

1994

KOEPKE, G. H.; RANDA, J.

Screen-Room Measurements on the NIST Spherical-Dipole Standard Radiator

NIST JRES, Vol. 99, No. 6, pp. 737-749

November/December 1994 
HILL, D. A.

Electronic Mode Stirring for Reverberation Chambers

IEEE Trans. Electromagn. Compat., Vol. 36, No. 4, pp. 294-299 November 1994

HILL, D. A.; CAVCEY, K. H.; JOHNK, R. T.

Crosstalk Between Microstrip Transmission Lines

IEEE Trans. Electromagn. Compat., Vol. 36, No. 4, pp. 314-321

November 1994

KANDA, M.

Standard Antennas for Electromagnetic Interference Measurements and Methods to Calibrate Them IEEE Trans. Electromagn. Compat., Vol. 36, No. 4, pp. 261-273

November 1994

CAMELL, D. G.; MA, M. T.

Assessment of Particular Measurement Data by a Linear Transfer Function Technique

NIST TN 1372

October 1994

HILL, D. A.; MA, M. T.; ONDREJKA, A. R.; RIDDLE, B. F.; CRAWFORD, M. L.; JOHNK, R. T. Aperture Excitation of Electrically Large, Lossy Cavities

IEEE Trans. Electromagn. Compat., Vol. 36, No. 3, pp. 169-178

August 1994

HILL, D. A.; CRAWFORD, M. L.; JOHNK, R. T.; ONDREJKA, A. R.; CAMELL, D. G.

Measurements of Shielding Effectiveness and Cavity Characteristics of Airplanes

NISTIR 5023

July 1994

JOHNK, R. T.; KANDA, M. T.

Alternative Contour Technique for the Efficient Computation of the Effective Length of an Antenna IEEE Trans. Antennas Propagat., Vol. 42, No. 5, pp. 747-749

May 1994

HILL, D. A.; CAVCEY, K. H.; JOHNK, R. T.

Crosstalk between Microstrip Transmission Lines

NISTIR 5015

December 1993

KANDA, M.

Standard Probes for Electromagnetic Field Measurements

IEEE Trans. Antennas Propagat., Vol. 41, No. 10, pp. 1349-1364

October 1993

RANDA, J.; KOEPKE, G. H.

Results of Screened-Room Measurements on NIST Standard Radiators

NISTIR 5010

October 1993 
HILL, D. A.; ADAMS, J. W.; MA, M. T.; ONDREJKA, A. R.; RIDDLE, B. F.; CRAWFORD, M. L.;

JOHNK, R. T.

Aperture Excitation of Electrically Large, Lossy Cavities

NIST TN 1361

September 1993

MA, M. T.; ADAMS, J. W.

Characterization of Unknown Linear Systems Based on Measured CW Amplitude

Record, IEEE Intl. Symp. on Electromagn. Compat., 9-13 August 1993, Dallas, TX, pp. 78-82 August 1993

NOVOTNY, D. R.; MASTERSON, K. D.; KANDA, M.

An Optically Linked Three-Loop Antenna System for Determining the Radiation Characteristics of an Electrically Small Source

Record, IEEE Intl. Symp. on Electromagn. Compat., 9-13 August 1993, Dallas, TX, pp. 300-304

August 1993

RANDA, J.; GILLILAND, D.; GJERTSON, W.; LAUBER, W.; McINERNEY, M.

Condensed Catalogue of Electromagnetic Environment Measurements, $30-300 \mathrm{~Hz}$

Record, IEEE Intl. Symp. on Electromagn. Compat., 9-13 August 1993, Dallas, TX, pp. 126-131

August 1993

MA, M. T.

Selected EMC Standards and Regulations: A Summary

NISTIR 5005

July 1993

MA, M. T.; ADAMS, J. W.

Characteristics of Unknown Linear Systems Deduced from Measured CW Magnitude

NIST JRES, Vol. 93, No. 3, pp. 297-319

May-June 1993

HILL, D. A.

Gradiometer Antennas for Tunnel Detection

Proc., 4th Tunnel Detection Symp. on Subsurface Exploration Technology, April 26-29, 1993,

Golden, $\mathrm{CO}$

April 1993

HILL, D. A.; CRAWFORD, M. L.; KANDA, M.

Aperture Coupling to Shielded Transmission Lines: Theory and Experiment

Proc. 10th Intl. Symp. on EMC, 9-11 March 1993, Zurich, Switzerland, pp. 569-571

March 1993

HILL, D. A.; CRAWFORD, M. L.; KANDA, M.; WU, D. I.

Aperture Coupling to a Coaxial Air Line: Theory and Experiment

IEEE Trans. Electromagn. Compat., Vol. 35, No. 1, pp. 69-74

February 1993

RANDA, J.

Correction Factor for Nonplanar Incident Field in Monopole Calibrations

IEEE Trans. Electromagn. Compat., Vol. 35, No. 1, pp. 94-96

February 1993 
HILL, D. A.

Electromagnetic Scattering by a Periodic Surface with a Wedge Profile

Electromagnetics, Vol. 12, pp. 247-264

1992

HILL, D. A.

Currents Induced on Multiconductor Transmission Lines by Radiation and Injection

IEEE Trans. Electromagn. Compat., Vol. 34, No. 4, pp. 445-450

November 1992

ADAMS, J. W.

Electromagnetic Shielding of RF Gaskets Measured by Two Methods

Record, 1992 IEEE Intl. Symp. on EMC, 17-21 August 1992, Anaheim, CA, pp. 154-157

August 1992

ADAMS, J. W.; CRUZ, J. E.; MELQUIST, D. G.

Comparison Measurements of Currents Induced by Radiation and Injection

IEEE Trans. Electromagn. Compat., Vol. 34, No. 3, pp. 360-362

August 1992

CRAWFORD, M. L.; RIDDLE, B. F.

A Reverberating Asymmetric TEM Cell for Radiated EMC/V and SE Testing, $10 \mathrm{kHz}-18 \mathrm{GHz}$

Record, 1992 IEEE Intl. Symp. on EMC, 17-21 August 1992, Anaheim, CA, pp. 206-213

August 1992

KOEPKE, G. H.; DRIVER, L. D.; CAVCEY, K. H.; MASTERSON, K. D.; JOHNK, R. T.; KANDA, M. New Spherical Dipole Source

Record, 1992 IEEE Intl. Symp. on EMC, 17-21 August 1992, Anaheim, CA, pp. 98-105 August 1992

RANDA, J.; KANDA, M.; ORR, R. D.

Optimized Thermo-Optic Electric-Field Probes for Microwaves and Millimeter Waves

Record, 1992 IEEE Intl. Symp. on EMC, 17-21 August 1992, Anaheim, CA, pp. 200-203

August 1992

TOFANI, S.; ONDREJKA, A. R.; KANDA, M.; HILL, D. A.

Bistatic Scattering of Absorbing Materials from 30 to $1000 \mathrm{MHz}$

IEEE Trans. Electromagn. Compat., Vol. 34, No. 3, pp. 304-307

August 1992

HILL, D. A.

Gradiometer Antennas for Tunnel Detection

NISTIR 3990

June 1992

MA, M. T.

EMC Standards and Regulations: A Brief Review

NISTIR 3989

May 1992

MA, M. T.; ADAMS, J. W.

System Response to Pulsed Excitations Estimated from Measurement of CW Amplitudes

Proc., Intl. Symp. on Electromagn. Compat., 25-27 May 1992, Beijing, China, pp. 29-32

May 1992 
HILL, D. A.; CRAWFORD, M. L.; KANDA, M.; WU, D. I.

Aperture Coupling to Shielded Transmission Lines: Theory and Experiment NISTIR 3988

April 1992

KANDA, M.; HILL, D. A.

A Three-Loop Method for Determining the Radiation Characteristics of an Electrically Small Source

IEEE Trans. Electromagn. Compat., Vol. EMC-34, No. 1, pp. 1-3

February 1992

KANDA, M.; MASTERSON, K. D.

Optically Sensed EM-Field Probes for Pulsed Fields

Proc., IEEE Spec. Issue on Antennas, Vol. 80, No. 1, pp. 209-215

January 1992

KOEPKE, G. H.; DRIVER, L. D.; CAVCEY, K. H.; MASTERSON, K. D.; JOHNK, R. T.; KANDA, M.

A Standard Spherical Dipole Source

NIST TN 1351

December 1991

MA, M. T.; LARSEN, E. B.; CRAWFORD, M. L.

Electromagnetic Fields with Arbitrary Wave Impedances Generated Inside a TEM Cell

IEEE Trans. Electromagn. Compat., Vol. EMC-33, No. 4, pp. 358-362

November 1991

MA, M. T.; ADAMS, J. W.

Phase Characteristics and Time Responses of Unknown Linear Systems Determined from Measured CW Amplitude Data

NIST TN 1349

November 1991

RANDA, J.; KANDA, M.; ORR, R. D.

Thermo-Optic Designs for Electromagnetic Field Probes for Microwaves and Millimeter Waves

IEEE Trans. Electromagn. Compat., Vol. EMC-33, No. 3, pp. 205-314

August 1991

RANDA, J.; KANDA, M.; ORR, R. D.

Resistively-Tapered-Dipole Electric-Field Probes up to $40 \mathrm{GHz}$

Proc., IEEE Intl. Electromagn. Compat. Symp. 13-15 August 1991 Cherry Hill, NJ, pp. 265-266

August 1991

HILL, D. A.

Diffraction by a Half Plane in a Lossy Medium

J. Appl. Phys., Vol. 69, No. 12, pp. 8405-8407

June 1991

ONDREJKA, A. R.; KANDA, M.

A Time-Domain Method for Measuring the Reflection Coefficient of Microwave Absorbers at Frequencies Below $1 \mathrm{GHz}$

Dig., 1991 Antenna Prop. Soc. Symp., 24-28 June 1991, London, Ontario, Canada, Vol. 3, pp. 1656-1663

June 1991 
CRAWFORD, M. L.; RIDDLE, B. F.

A Proposed TEM Driven Mode-Stirred Chamber for Large System Radiated EMC/V Testing $10 \mathrm{kHz}-40$ $\mathrm{GHz}$

Proc., 9th Intl. Symp. on Electromagn. Compat. 12-14 March 1991, Zurich, Switzerland, pp. 431-437

March 1991

\section{KANDA, M.; RANDA, J.}

Estimation of Electromagnetic Fields in Complex Environments

Proc., 9th Intl. Symp. on Electromagn. Compat., 12-14 March 1991, Zurich, Switzerland, pp. 337-342

March 1991

RANDA, J.

Simultaneous vs. Independent Injection Testing of Nonlinear Multiport Systems

Proc., 9th Intl. Symp. on Electromagn. Compat., 12-14 March 1991, Zurich, Switzerland, pp. 71-74

March 1991

HILL, D. A.

A Generalization of the Cornu Spiral for Lossy Media

J. Appl. Phys., Vol. 69, No. 3, pp. 1772-1774

February 1991

WILSON, P. F.; MA, M. T.

Fields Radiated by Electrostatic Discharges

IEEE Trans. Electromagn. Compat., Vol. EMC-33, No. 1, pp. 10-18

February 1991

CAMELL, D. G.; LARSEN, E. B.; CRUZ, J. E.; HILL, D. A.

NIST Calibration Procedure for Vertically Polarized Monopole Antennas, $30 \mathrm{kHz}$ to $300 \mathrm{MHz}$ NIST TN 1347

January 1991

RANDA, J.

Theoretical Considerations for a Thermo-Optic Microwave Electric-Field Probe

J. Microwave Power Electromagn. Energy, Vol. 25, No. 3, pp. 133-140

1990

HILL, D. A.

Near-Field and Far-Field Excitation of a Long Conductor in a Lossy Medium

NISTIR 3954

September 1990

CRAWFORD, M. L.; MA, M. T.; LADBURY, J. M.; RIDDLE, B. F.

Measurement and Evaluation of a TEM/Reverberating Chamber

NIST TN 1342

July 1990

HILL, D. A.

Quasi-Static Analysis of a Two-Wire Transmission Line Located at an Interface

Radio Sci., Vol. 25, No. 4, pp. 435-440

July-August 1990 
CRAWFORD, M. L.; LADBURY, J. M.; RIDDLE, B. F.; LARSEN, E. B.

Electromagnetic Radiation Test Facilities: Evaluation of a Small Reverberating Chamber Located at RADC, Griffiss AFB, Rome, NY

NISTIR 90-3939

June 1990

MASTERSON, K. D.; KANDA, M.

Broadband, Photonic Electric Field Sensors for EMP and HPM Applications

Proc., Fifth Natl. Conf. on High Power Microwave Technology (DoD), 10-15 June 1990, West Point, NY, pp. 388-391

June 1990

ADAMS, J. W.; WU, D. I.; BUDLONG, A.

Measurement of Electric Field Strength Near Higher-Powered Personal Transceivers

NISTIR 90-3938

May 1990

HILL, D. A.; KANDA, M.; LARSEN, E. B.; KOEPKE, G. H.; ORR, R. D.

Generating Standard Reference Electromagnetic Fields in the NIST Anechoic Chamber, 0.2 to $40 \mathrm{GHz}$ NIST TN 1335

March 1990

KANDA, M.

A Microstrip Patch Antenna as a Standard Transmitting and Receiving Antenna.

IEEE Trans. Electromagn. Compat., Vol. 32, No. 1, pp. 5-8

February 1990

MA, M. T.; CRAWFORD, M. L.

Facilities for Improving Evaluations of Electromagnetic Susceptibilities of Weapon Systems and

Electronic Equipment

NISTIR 89-3928

November 1989

WU, D. I.; KANDA, M.

Comparison of Theoretical and Experimental Data for the Near-Field of an Open Ended Rectangular Waveguide

IEEE Trans. Electromagn. Compat., Vol. EMC-31, No. 4, pp. 353-358

November 1989

KANDA, $M$.

A Microstrip Patch Antenna as a Standard Transmitting and Receiving Antenna

Dig., 1989 Intl. Symp. on Electromagn. Compat., 8-10 September 1989, Nagoya, Japan, pp. $460-462$

September 1989

MASTERSON, K. D.; DRIVER, L. D.

A Broadband, Isotropic, Photonic Electric Field Probe for Measurements from $10 \mathrm{kHz}$ to Above $1 \mathrm{GHz}$ Proc., High Bandwith Analog Applications of Photonics II, SPIE - Intl. Soc. of Optical

Engineers, Vol. 987 8-9 September 1988, Boston, MA, pp. 107-118

September 1989 
CRAWFORD, M. L.

TEM/Reverberating Chamber Design/Concept Study: A Single Facility for Large System Radiated EMC Testing, $10 \mathrm{kHz}-40 \mathrm{GHz}$ ?

Proc., EMC EXPO, 1-3 August 1989, Washington, DC, pp. B6.22-B6.29 August 1989

FITZGERRELL, R. G.

Monopole Impedance and Gain Measurements of Finite Ground Planes

National Institute of Justice Report, 200-87

May 1989

LARSEN, E. B.; EHRET, R. L.; CAMELL, D. G.; KOEPKE, G. H.

Calibration of Antenna Factor at a Ground Screen Field Site Using an Automatic Network Analyzer Proc., IEEE 1989 Natl. Symp. on Electromagn. Compat., 23-25 May 1989, Denver, CO, pp. 19-24

May 1989

MASTERSON, K. D.; DRIVER, L. D.; KANDA, M.

Photonic Probes for the Measurement of Electromagnetic Fields over Broad Bandwidths Proc., IEEE 1989 Natl. Symp. on Electromagn. Compat., 23-25 May 1989, Denver, CO, pp. 1-6

May 1989

RANDA, J.; KANDA, M.; MELQUIST, D. G.; ORR, R. D.

Thermo-optic Designs for Microwave and Millimeter-wave Electric-Field Probes Proc., IEEE 1989 Natl. Symp. on Electromagn. Compat., 23-25 May 1989, Denver, CO, pp. 7-11 May 1989

WU, D. I.; CHANG, D. C.

The Effect of an Electrically Large Stirrer in a Mode-Stirred Chamber IEEE Trans. Electromagn. Compat., Vol. EMC-31, No. 2, pp. 164-169 May 1989

KOEPKE, G. H.; MA, M. T.; BENSEMA, W. D.

Implementation of an Automated System for Measuring Radiated Emissions Using a TEM cell IEEE Trans. Instrum. Meas., Vol. IM-38, No. 2, pp. 473-479 April 1989

CRUZ, J. E.; LARSEN, E. B.

Alternative Techniques for Some Typical MIL-STD-461/462 Types of Measurements NIST TN 1320

March 1989

MA, M. T.

How High is the Level of Electromagnetic Fields Radiated by an ESD?

Proc., 8th Intl. Symp. on Electromagn. Compat., 7-9 March 1989, Zurich, Switzerland, pp. 361-365

March 1989 
CAVCEY, K. H.

Transmission Loss Through 6061 T-6 Aluminum Using a Pulsed Eddy Current Source

"Materials Evaluation," American Society for Nondestructive Testing, Vol. 47, No. 2, pp. 216-218

February 1989

HILL, D. A.

Clutter Models for Subsurface Electromagnetic Applications

NISTIR 89-3909

February 1989

HILL, D. A.

Reflection Coefficient of a Waveguide with Slightly Uneven Walls

IEEE Trans. Microwave Theory Tech., Vol MTT-37, No. 1, pp. 244-252 January 1989

KOEPKE, G. H.; MA, M. T.; BENSEMA, W. D.

Theory and Measurements of Radiated Emissions Using a TEM Cell

NIST TN 1326

January 1989

ADAMS, J. W.; FRIDAY, D. S.

Measurement Procedures for Electromagnetic Compatibility Assessment of Electroexplosive Devices

IEEE Trans. Electromagn. Compat., Vol. EMC-30, No. 4, pp. 484-494

November 1988

DRIVER, L. D.; KANDA, M.

An Optically Linked Electric and Magnetic Field Sensor for Poynting Vector Measurements in the Near Fields of Radiating Sources

IEEE Trans. Electromagn. Compat., Vol. EMC-30, No. 4, pp. 495-503

November 1988

REEVE, G. R.

Proficiency Testing for MIL-STD 462 NVLAP Laboratories

Dig., EMC EXPO '88, 10-12 May 1988, Washington, DC, pp. T33.13 through T33.15

September 1988

WU, D. I.; CHANG, D. C.

A. Hybrid Representation of the Green's Function in an Over-Moded Rectangular Cavity

IEEE Trans. Microwave Theory Tech., Vol. MTT-36, No. 9,pp. 1334-1342

September 1988

CAMELL, D. G.; LARSEN, E. B.; ANSON, W. J.

NBS Calibration Procedures for Horizontal Dipole Antennas (25 to $100 \mathrm{MHz}$ )

Proc. IEEE Intl. Symp. on Electromagn. Compat., 2-4 August 1988, Seattle, WA, pp. 390-394 August 1988

CRAWFORD, M. L.; LADBURY, J. M.

Mode-Stirred Chamber for Measuring Shielding Effectivess of Cables and Connectors an Assessment of MIL-STD 1344A Method 3008

Proc., 1988 IEEE Intl. Symp. on Electromagn. Compat., 1-5 August 1988, Seattle, WA, pp. 30-36

August 1988 
JESCH, R. L.

Measurement of Shielding Effectiveness of Different Cable and Shielding Configurations by Mode-Stirred Techniques

IEEE Trans. Electromagn. Compat., Vol. EMC-30, pp. 222-228

August 1988

KANDA, M.; ORR, R. D.

Generation of Standard Electromagnetic Fields in a TEM Cell

NBS TN 1319

August 1988

WILSON, P. F.; MA, M. T.

Techniques for Measuring the Electromagnetic Shielding Effectiveness of Materials: Part II - Near-Field Source Simulation

IEEE Trans. Electromagn. Compat., Vol. EMC-30, No. 3, pp. 251-259

August 1988

WILSON, P. F.; MA, M. T.; ADAMS, J. W.

Techniques for Measuring the Electromagnetic Shielding Effectivenss of Materials: Part I - Far-Field

Source Simulation

IEEE Trans. Electromagn. Compat., Vol. EMC-30, No. 3, pp. 239-250 August 1988

WILSON, P. F.; MA, M. T.; ONDREJKA, A. R.

Fields Radiated by Electrostatic Discharges

Proc., IEEE Intl. Symp. on Electromagn. Compat., 2-4 August 1988, Seattle, WA, pp. 179-183 August 1988

KANDA, M.; DRIVER, L. D.

An Optically Linked Electric and Magnetic Field Sensor for Poynting Vector Measurements in the Near Field of Radiating Sources

Dig., Conf. on Prec. Electromagn. Meas., 7-10 June 1988, Tsukuba Science City, Japan, pp. 32-33

June 1988

MA, M. T.

Theory and Measurements of Unintentional Radiators

Dig., Conf. on Prec. Electromagn. Meas., 7-10 June 1988, Tsukuba Science City, Japan, pp. 30-31

June 1988

RANDA, J.; KANDA, M.; MELQUIST, D. G.; SEGA, R. M.; NORGARD, J. D.

High Frequency Electric Field Probe Development

Proc., EMC EXPO '88, Intl. Conf. on Electromagn. Compat., 10-12 May 1988, Washington, DC, pp. T15.31 through T15.37

May 1988

VANZURA, E. J.

Automated System for Electromagnetic Field Generation and Immunity Testing

Proc., 1988 IEEE Instrum. Meas. Technol. Conf., 19-22 April 1988, San Diego, CA, pp. 3-10 April 1988 
FITZGERRELL, R. G.

Monopole Impedance and Gain Measurements on Finite Ground Planes

IEEE Trans. Antennas Propagat., Vol. 36, No. 3, pp. 431-438 March 1988

MA, M. T.

Understanding Reverberating Chambers as an Alternative Facility for EMC Testing

J. Electromagn. Waves Appl., Vol. 2, pp. 339-351

March/April 1988

WU, D. I.; CHANG, D. C.

The Effect of a Large Rotating Scatterer in a Rectangular Cavity

NBS TN 1317

March 1988

HILL, D. A.

A Circular Array for Plane-Wave Synthesis

IEEE Trans. Electromagn. Compat., Vol. EMC-30, No. 1, pp. 3-8

February 1988

RANDA, J.; KANDA, M.; MELQUIST, D. G.

Possible Designs for Electric-Field Strength-Probes for Millimeter Waves

NBSIR 88-3084

February 1988

WILSON, P. F.; ONDREJKA, A. R.; MA, M. T.; LADBURY, J. M.

Electromagnetic Fields Radiated From Electrostatic Discharges - Theory and Experiment

NBS TN 1314

February 1988

WILSON, P. F.

A Comparison Between Near-Field Shielding Effectiveness Measurements Based on Coaxial Dipoles and on Electrically Small Apertures

IEEE Trans. Electromagn. Compat., Vol. EMC-30, No. 1, pp. 23-28

February 1988

CAVCEY, K. H.; FRIDAY, D. S.

Aircraft Field Degradation and Electromagnetic Compatibility

NBSIR 88-3083

January 1988

HILL, D. A.

Electromagnetic Detection of Long Conductors in Tunnels

Proc., Third Tunnel Detection Symp., 12-15 January 1988, Golden, CO, pp. 518-537

January 1988

HILL, D. A.

Effect of a Thin Conducting Sheet on the Fields of a Buried Magnetic Dipole

Electromagnetics, pp. 71-79

1987 
CRAWFORD, M. L.; KOEPKE, G. H.; LADBURY, J. M.

EMR Test Facilities Evaluation of Reverberating Chamber Located at RADC, Griffiss, AFB, Rome, New York

NBSIR 87-3080

December 1987

RANDA, J.; KANDA, M.

A New Approach to Volumes Irradiated by Unknown Sources

IEEE Trans. Electromagn. Compat., Vol. EMC-29, No. 4, pp. 273-281 November 1987

VANZURA, E. J.; ADAMS, J. W.

Generating Constant Electromagnetic Fields Inside a Partially-Loaded Shielded Room

Test and Meas. World, pp. 72-83

November 1987

JESCH, R. L.

Measurement of Shielding Effectiveness of Different Cable and Shielding Configurations by

Mode-Stirred Techniques

NBSIR 87-3076

October 1987

MA, M. T.

Characterization of Unknown RF Leakage Sources: Problems, Solutions and Practical Implications

Union Radio Scientifique Internationale XXII General Assembly, 24 August - 2 September

1987, Tel Aviv, Israel

September 1987

WU, D. I.; CHANG, D. C.

An Investigation of a Ray-Mode Representation of the Green's Function in a Rectangular Cavity NBS TN 1312

September 1987

CRAWFORD, M. L.

A TEM Driven Reverberating Chamber: A Single Facility for Radiated EMS/V Testing, $10 \mathrm{kHz}-18 \mathrm{GHz}$ ?

Proc., EMC Expo 1987, Intl. Conf. on Electromagn. Compat.,19-21 May 1987, San Diego, CA, pp. T11.18 through T11.28

May 1987

MA, M. T.; BENSEMA, W. D.

Automated TEM Cell for Measuring Unintentional EM Emissions

Proc., EMC Expo 1987, Intl. Conf. on Electromagn. Compat., 19-21 May 1987, San Diego, CA, pp. T11.1 through T11.12 May 1987

CRAWFORD, M. L.; KOEPKE, G. H.

Performing EM Susceptibility/Vulnerability Measurements Using a Reverberation Chamber

Proc., 7th Intl. Symp. on Electromagn. Compat., 3-5 March 1987, Zurich, Switzerland, pp. 121-126

March 1987 
MLARTZLOFF, F. D.; WILSON, P. F.

Fast Transient Tests - Trivial or Terminal Pursuit?

Proc., 7th Intl. Symp. on Electromagn. Compat., 3-5 March 1987, Zurich, Switzerland, pp. 283-288

March 1987

RANDA, J.; KANDA, M.

A Lattice Approach to Environments Irradiated by Unknown Sources

Proc., 7th Intl. Symp. Electromagn. Compat., 3-5 March 1987, Zurich, Switzerland, pp. 191-195 March 1987

WILSON, P. F.; MA, M. T.

Techniques for Measuring the Shielding Effectiveness of Materials

Proc., 7th Intl. Symp. on Electromagn. Compat., 3-5 March 1987, Zurich, Switzerland, pp. 547-552

March 1987

KANDA, M.; DRIVER, L. D.

An Isotropic, Electric-Field Probe with Tapered Resistive Dipoles for Broadband Use, $100 \mathrm{kHz}-18 \mathrm{GHz}$

IEEE Trans. Microwave Theory Tech., Vol. MTT-35, No. 2, pp. 124-130 February 1987

HILL, D. A.

Radio-Wave Propagation from a Forest to a Clearing

Electromagnetics, pp. 217-228

1986

\section{LARSEN, E. B.}

Calibration and Meaning of Antenna Factor and Gain for EMI Antennas

Interference Technology Engineers' Master (ITEM 1986), pp. 113-335 1986

\section{MASTERSON, K. D.}

A Photonic Electric Field Probe for Frequencies up to $2 \mathrm{GHz}$

Proc., Soc. for Photo-Optical Instrumentation Engineers (SPIE), Vol. 720, p. 100 1986

CRAWFORD, M. L.; BEAN, J. L.

NSWC Reverberating Chamber: A High Power Microwave Exposure Chamber

Proc., 3rd Natl. High Power Microwave Technical Conf., 1-5 December 1986, Kirtland AFB, NM, p. 5

December 1986

HILL, D. A.; KOEPKE, G. H.

A Near-Field Array of Yagi-Uda Antennas for Electromagnetic Susceptibility Testing

IEEE Trans. Electromagn. Compat., Vol. EMC-28, No. 4, pp. 273-276

November 1986

HILL, D. A.

An Error Bound for Near-Field Array Synthesis

IEEE Trans. Electromagn. Compat., Vol. EMC-28, No. 4, pp. 273-276

November 1986 
BENSEMA, W. D.; KOEPKE, G. H.; MEDLEY, H. W.

Handbook for NBS Multisensor Automated EM Field Measurement System NBSIR 86-3056

October 1986

CRUZ, J. E.; LARSEN, E. B.

Assessment of Errors for MIL-STD-461/462

NBS TN 1300

October 1986

JESCH, R. L.

A Survey of Triaxial and Mode-Stirred Techniques for Measuring the Shielding Effectiveness of Connectors and Cables

NBSIR 86-3060

October 1986

KANDA, M.; WYSS, J. C.

Evaluation of Off-Axis Measurements Performed in an Anechoic Chamber

NBS TN 1305

October 1986

RANDA, J.; KANDA, M.

A Lattice Approach to Volumes Irradiated by Unknown Sources

NBS TN 1303

October 1986

ADAMS, J. W.; VANZURA, E. J.

Shielding Effectiveness Measurements of Plastics

EMC Technology and Interference Control News, pp. 39-44

September-October 1986

CRAWFORD, M. L.; KOEPKE, G. H.

Preliminary Evaluation of Reverberation Chamber Method for Pulsed RF Immunity Testing Record, IEEE Intl. Symp. on Electromagn. Compat., 16-18 September 1986, San Diego, CA, pp. $270-278$

September 1986

\section{KANDA, M.; DRIVER, L. D.}

An Isotropic, Electric-Field Probe with Tapered Resistive Dipoles for Broadband Use, $100 \mathrm{kHz}-18 \mathrm{GHz}$

Record, IEEE Intl. Symp. on Electromagn. Compat., 16-18 September 1986, San Diego, CA, pp. 256-261

$$
\text { September } 1986
$$

KANDA, M.; ORR, R. D.

Near-Field Gain of a Horn and an Open-Ended Waveguide: Comparison Between Theory and Experiment

Proc., 5th Intl. Conf. on Electromagn. Compat., 29 September-2 October 1986, University of York, England, pp. 137-145

September 1986 
MA, M. T.; KANDA, M.; CRAWFORD, M. L.; LARSEN, E. B.

Measuring Electromagnetic Interference, Part I, Open-Field Sites and TEM Cells; Part II, Reverberating Chambers; Part III, Anechoic Chambers and Field Probes

Test and Measurement World, Part I, pp. 72-88, February 1986; Part II, pp. 74-90, May 1986,

Part III, pp. 81-99, September 1986

September 1986

RANDA, J.; KANDA, M.

A Lattice Approach to Complex Electromagnetic Environments

Record, IEEE Intl. Symp. on Electromagn. Compat., 16-18 September 1986, San Diego, CA, pp. 329-331

September 1986

WILSON, P. F.; MA, M. T.

Shielding Effectiveness Measurements Using an Apertured TEM Cell in a Reverberation Chamber Record, IEEE Intl. Symp. on Electromagn. Compat., 16-18 September 1986, San Diego, CA, pp. 265-269

September 1986

HILL, D. A.

Out-of-Band Response of a Coax-to-Waveguide Adapter

IEEE Trans. Electromagn. Compat., Vol. EMC-28, No. 3, pp. 156-158

August 1986

WILSON, P. F.; MA, M. T.

Simple Approximate Expressions for Higher-Order Mode Cut Off and Resonant Frequencies in TEM Cells

IEEE Trans. Electromagn. Compat., Vol. EMC-28, No. 3, pp. 125-130

August 1986

HILL, D. A.; WAIT, J. R.

Anomalous Vertical Magnetic Field for Electromagnetic Induction in a Laterally Varying Thin Conductive Sheet

Radio Sci., Vol. 21, No. 4, pp. 617-621

July-August 1986

MA, M. T.; KANDA, M.

Electromagnetic Compatibility and Interference Metrology

NBS TN 1099

July 1986

CRAWFORD, M. L.; KOEPKE, G. H.

Performing EM Susceptibility/Vulnerability Measurements Using a Reverberation Chamber Proc., EMC EXPO 1986, Intl. Conf. on Electromagn. Compat., 16-19 June 1986, Washington, DC, pp. T28.7 through T28.14

June 1986

CRAWFORD, M. L.; KOEPKE, G. H.

EMR Test Facilities - Evaluation of Reverberation Chambers Located at NSWC, Dahlgren, VA NBSIR 86-3051

June 1986 
KANDA, M.; DRIVER, L. D.

A Broadband, Electric-Field Probe Using Resistively Tapered Dipoles, $100 \mathrm{kHz}-18 \mathrm{GHz}$

Proc., 1986 IEEE/MTT/S Intl. Microwave Symp. Dig., 2-4 June 1986, Baltimore, MD, pp. 621-624

June 1986

REEVE, G. R.

Altemate EMI Measurement Techniques for Microelectronic Circuits

Proc., EMC EXPO 1986, Intl. Conf. on Electromagn. Compat., 16-19 June 1986, Washington, DC, pp. T26.1 through T26.4

June 1986

WILSON, P. F.; MA, M. T.

Methods for Measuring the Near-Field and Far-Field Shielding Effectiveness of Materials

Proc., EMC Expo 1986 Intl. Conf. on Electromagn. Compat., 16-19 June 1986, Washington,

DC, pp. T28.1-T28.6

June 1986

FRIDAY, D. S.; ADAMS, J. W.

A Statistical Characterization of Electroexplosive Devices Relevant to Electromagnetic Compatibility NBS TN 1094

May 1986

JESCH, R. L.

Susceptibility of Emergency Vehicle Sirens to External Radiated Electromagnetic Fields

National Institute of Justice Technology Program, NIJ Report-200-85

May 1986

WILSON, P. F.; MA, M. T.

A Study of Techniques for Measuring the Electromagnetic Shielding Effectiveness of Materials

NBS TN 1095

May 1986

CRAWFORD, M. L.; KOEPKE, G. H.

Design, Evaluation and Use of a Reverberation Chamber for Performing Electromagnetic

Susceptibility/Vulnerability Measurements

NBS TN 1092

April 1986

KUFFEL, J.; MALEWSKI, R.; VAN HEESWIJK, R.; LAWTON, R. A.

Dynamic Performance of Digital Recorders Used for Monitoring High-Voltage Impulse Tests

Proc., IEEE Instrum. Meas. Technol. Conf., 25-27 March 1986, Boulder, CO, Vol. IM-35, No.

4, pp. 591-595

March 1986

FITZGERRELL, R. G.

Site Attenuation

IEEE Trans. Electromagn. Compat., Vol. EMC-28, No. 1, pp. 38-40

February 1986

HILL, D. A.

Electromagnetic Wave Propagation in an Asymmetrical Coal Seam

IEEE Trans. Antennas Propagat., Vol. AP-34, No. 2, pp. 244-247

February 1986 
WILSON, P. F.; MA, M. T.

Electromagnetic Shielding Effectiveness: Measurement Techniques and Interpretations

1986 IEEE Regional Conf. and Exhib. on Electromagn. Compat.,6 February 1986, Anaheim, CA, pp. 1-14

February 1986

ADAMS, J. W.; VANZURA, E. J.

Shielding Effectiveness Measurements of Plastics

NBSIR 85-3035

January 1986

FRIDAY, D. S.

Methodology for Statistical Control of the Anechoic Chamber Field Generation System

NBSIR 85-3033

January 1986

KANDA, M.; LARSEN, E. B.; BORSERO, M.; GALLIANO, P. G.; YODOSHIMA, I.; NAHMAN, N. S. Standards for Electromagnetic Fields Measurements

Proc., IEEE Spec. Issue on Radio Meas. Methods and Standards, Vol. 74, No. 1, pp. 120-128 January 1986

MA, M. T.

Measurements of Unintentional Electromagnetic Emissions

Proc., IEEE Spec. Issue on Radio Meas. Methods and Standards, Vol. 74, No. 1, pp. 110-111 January 1986

WILSON, P. F.; MA, M. T.

Measurements of Electromagnetic Shielding Capabilities of Materials

Proc., IEEE Spec. Issue on Radio Meas. Methods and Standards, Vol. 74, No. 1, pp. 112-115 January 1986

NAHMAN, N. S.; KANDA, M.; LARSEN, E. B.; CRAWFORD, M. L.

Methodology for Standard Electromagnetic Field Measurements

IEEE Trans. Instrum. Meas., Vol. IM-34, No. 4, pp. 490-503

December 1985

FITZGERRELL, R. G.

Site Attenuation

NBS TN 1089

November 1985

HILL, D. A.

A Numerical Method for Near-Field Array Synthesis

IEEE Trans. Electromagn. Compat., Vol. EMC-27, No. 4, pp. 201-211

November 1985

CRUZ, J. E.; DRIVER, L. D.; KANDA, M.

Design of the National Bureau of Standards Isotropic Magnetic Field Meter (MFM-10) $300 \mathrm{kHz}$

to $100 \mathrm{MHz}$

NBS TN 1085

October 1985 
WILSON, P. F.; CHANG, D. C.

Mode Coupling by a Longitudinal Slot for a Class of Planar Waveguiding Structures: Part I - Theory IEEE Trans. Microwave Theory Tech., Vol. MTT-33, No. 10, pp. 981-987 October 1985

WILSON, P. F.; CHANG, D. C.

Mode Coupling by a Longitudinal Slot for a Class of Planar Waveguiding Structures: Part II Applications

IEEE Trans. Microwave Theory Tech., Vol. MTT-33, No. 10, pp. 988-993 October 1985

CRAWFORD, M. L.; KOEPKE, G. H.

Comparing EM Susceptibility Measurement Results Between Reverberation and Anechoic Chambers Record, IEEE 1985 Intl. Symp. on Electromagn. Compat., 20-22 August 1985, Wakefield, MA, pp. 200-202

August 1985

FITZGERRELL, R. G.

Site Attenuation

Record, IEEE 1985 Intl. Symp. on Electromagn. Compat., 20-22 August 1985, Wakefield, MA, pp. $612-617$

August 1985

HILL, D. A.; KOEPKE, G. H.

An Array of Dipoles for Plane Wave Synthesis

Proc., 1985 Intl. Symp. on Antennas and Propagat.,20-22 August 1985, Kyoto, Japan, pp. $177-180$

August 1985

KANDA, M.; ORR, R. D.

A Radio-Frequency Power Delivery System: Procedures for Enor Analysis and Self-Calibration NBS TN 1083

$$
\text { August } 1985
$$

RANDA, J.; KANDA, M.

Directional Scanning of Complex Electromagnetic Environments

Proc., 1985 Intl. Symp. on Antennas and Propagat., 20-22 August 1985, Kyoto, Japan, pp. $899-902$

\section{August 1985}

\section{RANDA, J.; KANDA, M.}

High Frequency Errors of an Electric-field-meter in Complicated Environments

Record, IEEE 1985 Intl. Symp. on Electromagn. Compat., 20-22 August 1985, Wakefield, MA, pp. 618-621

\section{August 1985}

RANDA, J.; KANDA, M.

A Finite-Element Action Approach to the Characterization of Complex Electromagnetic Environments Proc., 1985 Intl. Symp. on Antennas and EM Theory, 26-28 August 1985, Beijing, China, pp. $48-53$

August 1985 
WILSON, P. F.; MA, M. T.

Shielding Effectiveness Measurements in a Dual TEM Cell

IEEE Trans. Electromagn. Compat., Vol. EMC-27, No. 3, pp. 137-142 August 1985

WILSON, P. F.; MA, M. T.

Factors Influencing Material Shielding Effectiveness Measurements

Record, IEEE 1985 Intl. Symp. on Electromagn. Compat., 20-22 August 1985, Wakefield, MA, pp. 29-33

August 1985

HILL, D. A.; KOEPKE, G. H.

A Near-field Array of Yagi-Uda Antennas for Electromagnetic Susceptibility Testing

NBS TN 1082

July 1985

RANDA, J.; KANDA, M.

A Directional Scanning Technique for Characterization of Complex Electromagnetic Environments

Dig., 1985 IEEE Antenna Propagat. Soc. Symp., 17-21 June 1985, University of British

Columbia, Vancouver, BC, Canada, pp. 521-524

June 1985

WYSS, J. C.; SHEERAN, S.

A Passive, Optical Modulator and Link for Antennas

IEEE J. Lightwave Technol., Vol. LT-3, No. 2, pp. 316-321

April 1985

BENSEMA, W. D.; REEVE, G. R.; KOEPKE, G. H.

A Multisensor Automated EM Field Measurement System

Proc., 1985 Instrum. and Meas. Technol. Conf., 20-22 March 1985, Tampa, FL, pp. 200-202 March 1985

CRUZ, J. E.; LARSEN, E. B.

Screenroom Measurements of Antenna Factors

Proc., 1985 Instrum. and Meas. Technol. Conf., 20-22 March 1985, Tampa, FL, p. 208

March 1985

KANDA, M.; NAHMAN, N.S.

Standards for Measurement of Electromagnetic Fields

Proc., 1985 Instrum. and Meas. Technol. Conf., 20-22 March 1985, Tampa, FL, pp. 20-23 March 1985

KANDA, M.; RANDA, J.; NAHMAN, N. S.

Possible Estimation Methodologies for Electromagnetic Field Distributions in Complex Environments

NBS TN 1081

March 1985

KANDA, M.

A Methodology for Evaluating Microwave Anechoic Chamber Measurements

Proc., 1985 Symp. and Technical Exhib. on Electromagn. Compat., 5-7 March 1985, Zurich,

Switzerland, pp. 69-74

March 1985 
LARSEN, E. B.; CRUZ, J. E.

$\mathrm{E}$ and $\mathrm{H}$ Fields in Transmission Lines and Coils for Susceptibility Testing, Probe Calibration, and RF Exposure Chambers

Proc., 1985 Instrum. and Meas. Technol. Conf., 20-22 March 1985, Tampa, FL, p. 199 March 1985

MA, M. T.; KANDA, M.; CRAWFORD, M. L.; LARSEN, E. B.

A Review of Electromagnetic Compatibility/Interference Measurement Methodologies

Proc., IEEE, Vol. 73, No. 3, pp. 388-411

March 1985

RANDA, J.; KANDA, M.

Multiple-Source, Multiple-Frequency Error of an Electric Field Meter

IEEE Trans. Antennas Propagat., Vol. AP-33, No. 1, pp. 2-9 January 1985

SHAFER, J. F.

Field Strength Levels in Vehicles Resulting from Communications Transmitters

National Institute of Justice Report, 200-83

1984

WILSON, P. F.; MA, M. T.

Input Impedance of a Probe Antenna in a TEM Cell

IEEE Trans. Electromagn. Compat., Vol. EMC-26, No. 4, pp. 154-161

November 1984

KANDA, M.

Error Analysis of Radiation Characteristics of an Unknown Interference Source Based on Power

Measurements

Proc., 1984 Intl. Symp. on Electromagn. Compat., 16-18 October 1984, Tokyo, Japan, pp. 39-44

October 1984

WILSON, P. F.; MA, M. T.

Small Aperture Analysis of the Dual TEM Cell and an Investigation of Test Object Scattering in a Single TEM Cell

NBS TN 1076

October 1984

WILSON, P. F.; MA, M. T.

Small Obstacle Loading in a TEM Cell

Proc., 1984 Intl. Symp. on Electromagn. Compat., 16-18 October 1984, Tokyo, Japan, pp. 30-35

October 1984

WYSS, J. C.; ANSON, W. J.; ORR, R. D.

Building Penetration Project

NBSIR 84-3009

September 1984

\section{ONDREJKA, A. R.; ADAMS, J. W.}

Shielding Effectiveness (SE) Measurement Techniques

Proc., 1984 EMI/RFI Technical Conf., The Soc. of Plastics Engineers, Inc., 18-20 June 1984,

Chicago, IL, pp. 19-26

June 1984 
WILSON, P. F.; MA, M. T.

Some Problems Associated with Interpreting Shielding Effectiveness Measurement Results

Proc., 1984 EMI/RFI Technical Conf., The Soc. of Plastics Eng., Inc., 18-20 June 1984, Chicago, IL, pp. 9-18

June 1984

FITZGERRELL, R. G.

A Source of $\mathrm{E}$ and $\mathrm{H}$ Fields for Antenna-Factor Calibration

IEEE Trans. Electromagn. Compat., Vol. EMC-26, No. 2, pp. 58-65

May 1984

CRAWFORD, M. L.; KOEPKE, G. H.

Operational Considerations of a Reverberation Chamber for EMC Immunity Measurements, Some

Experimental Results

Proc., 1984 IEEE Natl. Symp. on Electromagn. Compat., 24-26 April 1984, San Antonio, TX, pp. 47-54

April 1984

DRIVER, L. D.; REEVE, G. R.

Application of a Systematic Approach to an Investigation of HF Interference to a Shipboard Radar Set Proc., 1984 IEEE Natl. Symp. on Electromagn. Compat., 24-26 April 1984, San Antonio, TX, pp. 211-218

April 1984

ONDREJKA, A. R.; ADAMS, J. W.

Shielding Effectiveness (SE) Measurement Techniques

Proc., 1984 IEEE Natl. Symp. on Electromagn. Compat., 24-26 April 1984, San Antonio, TX, pp. 249-256

April 1984

WILSON, P. F.; MA, M. T.

Small Aperture Analysis of the Dual TEM Cell

Proc., 1984 IEEE Natl. Symp. on Electromagn. Compat., 24-26 April 1984, San Antonio, TX, pp. 365-369

April 1984

HILL, D. A.

Theory of Near-Field Phased Arrays for Electromagnetic Susceptibility Testing NBS TN 1072

February 1984

ALSPACH, W. J.; MLLER, C. K. S.; REEVE, G. R.

An Assessment of Electromagnetic Interference (EMI) and Electromagnetic Compatibility (EMC) Measurement Practices to Meet U.S. Army Aviation Research and Development Command EMIEMC Requirements

NBSIR 84-1698 (Restricted)

January 1984

KANDA, M.

Arrays of Discrete Elements

Antenna Engineering Handbook, Chapter 3, R. C. Johnson and H. Jasik, eds., McGraw-Hill

Book Co.

January 1984 


\section{CRAWFORD, M. L.}

Comparison of Open-Field, Anechoic Chamber and TEM Cell Facilities/Techniques for Performing Electromagnetic Radiated Emissions Measurements

Record, IEEE 1983 Intl. Symp. on Electromagn. Compat., 23-25 August 1983, Arlington, VA, pp. $413-418$

August 1983

FITZGERRELL, R. G.

E-Fields Over Ground

Record, IEEE 1983 Intl. Symp. on Electromagn. Compat., 23-25 August 1983, Arlington, VA, pp. 6-9

August 1983

LIU, B. H.; CHANG, D. C.; MA, M. T.

Design Consideration of Reverberating Chambers for Electromagnetic Interference Measurements

Record, IEEE 1983 Intl. Symp. on Electromagn. Compat., 23-25 August 1983, Arlington, VA, pp. 508-512

$$
\text { August } 1983
$$

LIU, B. H.; CHANG, D. C.; MA, M. T.

Eigenmodes and the Composite Quality Factor of a Reverberating Chamber NBS TN 1066

August 1983

WILSON, P. F.; CHANG, D. C.; MA, M. T.; CRAWFORD, M. L.

Theoretical and Experimental Analysis of Coupling Characteristics of Dual TEM Cells

Record, IEEE 1983 Intl. Symp. on Electromagn. Compat., 23-25 August 1983, Arlington, VA, pp. 513-517

August 1983

BENSEMA, W. D.

Handbook for Broadband Isotropic Antenna System

Volume 1 - Operations Manual NBSIR 83-1693

July 1983

MA, M. T.; KOEPKE, G. H.

Uncertainties in Extracting Radiation Parameters for an Unknown Interference Source Based on Power and Phase Measurements

NBS TN 1064

June 1983

KANDA, M.; RIES, F. X.

Time Domain Sensors for Radiated Impulsive Measurements

IEEE Trans. Antennas Propagat., Vol. AP-31, No. 3, pp. 438-444

May 1983

KANDA, M.

An Electric and Magnetic Field Sensor for Simultaneous Electromagnetic Near-Field MeasurementsTheory

NBS TN 1062

April 1983 
CRAWFORD, M. L.

Evaluation of Shielded Enclosure for EMI/EMC Measurements Without and With RF Anechoic Material Proc., 1983 Electromagn. Compat. Symp. and Exhib., 8-10 March 1983, Zurich, Switzerland, pp. 397-402

March 1983

CRAWFORD, M. L.

Improving the Repeatability of EM Susceptibility Measurements of Electronic Components When Using TEM Cells

SAE Technical Paper Series, 830607, International Congress and Exposition, 28 February-

4 March 1983, Detroit, MI, pp. 1-8

March 1983

KANDA, M.; RIES, F. X.; DRIVER, L. D.; ORR, R. D.

An Electric and Magnetic Field Sensor Concept for Simultaneous Near-Field Electromagnetic

Components When Using TEM Cells

Proc., 1983 Electromagn. Compat. Symp. and Exhib., 8-10 March 1983, Zurich, Switzerland, pp. 263-266

March 1983

KOEPKE, G. H.; MA, M. T.

A New Method for Determining the Emission Characteristics of an Unknown Interference Source

Proc., 1983 Electromagn. Compat. Symp. and Exhib., 8-10 March 1983, Zurich, Switzerland, pp. 263-266

March 1983

STUBENRAUCH, C. F.; SPIESS, W.; GALLIANO, P. G.; BABJI, T.

International Intercomparison of Electric Field Strength at $100 \mathrm{MHz}$

IEEE Trans. Instrum. Meas., Vol. IM-32, No. 1, pp. 235-237

March 1983

MILLER, C. K. S.

The EMI Measurement Challenge

Proc., 1983 Meas. Sci. Conf., 20-21 January 1983, Palo Alto, CA, pp. 189-197

January 1983

FITZGERRELL, R. G.

A Partial Loop Source of E and H Fields for Antenna Factor Calibration (A Loop Cell)

Proc., 1982 Antenna Meas. Techniques Assoc., 5-7 October 1982, New Mexico State

University, Las Cruces, NM, pp. 15-1 through 15-22

October 1982

MA, M. T.; KOEPKE, G. H.

A Method to Quantify Radiation Characteristics of an Unknown Interference Source NBS TN 1059

October 1982

TAGGART, H. E.

Radiated EMI Instrumentation Errors

EMC Technology Magazine, Vol. 1, No. 4, pp. 26-35

October 1982 
DRIVER, L. D.; CRUZ, J. E.

Development of the NBS Isotropic Magnetic-Field Meter (MFM-10), $300 \mathrm{kHz}$ to $100 \mathrm{MHz}$

Proc., 1982 Intl. Symp. on Electromagn. Compat., 8-10 September 1982, Santa Clara, CA, pp. $460-467$

September 1982

KANDA, M.; RIES, F. X.

Time Domain Sensors for Radiated Impulsive Measurements

Proc., IEEE 1982 Intl. Symp. on Electromagn. Compat., 8-10 September 1982, Santa Clara, CA, pp. 296-301

September 1982

KOEPKE, G. H.; MA, M. T.

A New Method for Determining the Emission Characteristics of an Unknown Interference Source

Proc., IEEE 1982 Intl. Symp. on Electromagn. Compat.,8-10 September 1982, Santa Clara, CA, pp. 151-156

September 1982

ADAMS, J. W.

Measurement of Electromagnetic Radiation from Electric Rail Cars

NBSIR 82-1669

August 1982

FITZGERRELL, R. G.

Free-Space Transmission Loss for Anechoic Chamber Performance Evaluation

IEEE Trans. Electromagn. Compat., Vol. EMC-24, No. 3, pp. 356-358

August 1982

KANDA, M.; RIES, F. X.; DRIVER, L. D.; ORR, R. D.

Design Considerations for Broadband Magnetic-Field Sensors

Dig., 1982 Conf. on Prec. Electromagn. Meas., 28 June-1 July 1982, Boulder, CO, pp. P-11

through P-13

June 1982

WYSS, J. C.; KANDA, M.; MELQUIST, D. G.; ONDREJKA, A. R.

Optical Modulator and Link for Broadband Antennas

Dig., 1982 Conf. on Prec. Electromagn. Meas., 28 June-1 July 1982, Boulder, CO, pp. P-16

through P-17

June 1982

KANDA, M.

The Effects of Resistive Loading on TEM Horns

IEEE Trans. Electromagn. Compat., Vol. EMC-24, No. 2, pp. 245-255

May 1982

ARTHUR, M. G.; ORR, R. D.; REEVE, G. R.

Planning Guidance for Future EMI Measurement Instrumentation

NBSIR 82-1662

April 1982

WILSON, P. F.; CHANG, D. C.; MA, M. T.

Input Impedance of a Probe Antenna Exciting a TEM Cell

NBS TN 1054

April 1982 
MA, M. T.; ARTHUR, M. G.

A Study of Distribution of Electromagnetic Fields Inside Buildings with Apertures Excited by an External Source

NBSIR 82-1659

February 1982

RIES, F. X.; MLLER, C. K. S.

Influence of Electromagnetic Interference on Electronic Devices Bulletin

OIML Seminar

December 1981

TAGGART, H. E.

Methods of Suppressing Automotive Interference

NBS SP 480-44

November 1981

BENSEMA, W. D.

Broadband Orthogonal Array Antenna System: Microprocessor Control and Computation

Record, "EMC '81," 1981 IEEE Intl. Symp. on Electromagn. Compat., 18-20 August 1981, Boulder, $\mathrm{CO}$

August 1981

CRUZ, J. E.

Design, Construction, and Calibration of the Broadband Electric Field Monitor (EFM-5)

Record, "EMC '81," 1981 IEEE Intl. Symp. on Electromagn. Compat., 18-20 August 1981,

Boulder, $\mathrm{CO}$

August 1981

FITZGERRELL, R. G.

Free-Space Transmission Loss for Anechoic Chamber Performance Evaluation

Record, "EMC '81," 1981 IEEE Intl. Symp. on Electromagn. Compat., 18-20 August 1981, Boulder, $\mathrm{CO}$

August 1981

LARSEN, E. B.

Background and Present Status of RF Probe Development at NBS

Record, "EMC '81," 1981 IEEE Intl. Symp. on Electromagn. Compat., 18-20 August 1981,

Boulder, $\mathrm{CO}$

August 1981

REEVE, G. R.

Synthesized Isotropic Pattern Antennas for EM Field Measurements

Record, "EMC '81," 1981 IEEE Intl. Symp. on Electromagn. Compat., 18-20 August 1981,

Boulder, $\mathrm{CO}$

August 1981

SREENIVASIAH, I.; CHANG, D. C.; MA, M. T.

Emission Characteristics of Electrically Small Radiating Sources from Tests Inside a TEM Cell

IEEE Trans. Electromagn. Compat., Vol. EMC-23, No. 3, pp. 113-121

August 1981 
SREENIVASIAH, I.; CHANG, D. C.; MA, M. T.

A Critical Study of Emission and Susceptibility Levels of Electrically Small Objects from Tests Inside a TEM Cell

Record, "EMC '81," 1981 IEEE Intl. Symp. on Electromagn. Compat., 18-20 August 1981, Boulder, $\mathrm{CO}$

$$
\text { August } 1981
$$

CLARK, H. E.

Requirements for an Effective National Nonionizing Radiation Measurement System

NBS SP 613

June 1981

REEVE, G. R.; WAINWRIGHT, A. E.

A Frequency Tracking, Tuned, Receiving Monopole

Record, IEEE/APS Symp., June 1981

June 1981

KANDA, M.

Theoretical and Experimental Investigations of Electromagnetic Field Distortion Due to a Perfectly

Conducting Rectangular Cylinder in a Transverse Electromagnetic Cell

NBS TN 1028

April 1981

MA, M. T.; CHANG, D. C.; SREENIVASIAH, I.

A Method of Determining the Emission and Susceptibility Levels of Electrically Small Objects Using a TEM Cell

NBS TN 1040

April 1981

CRAWFORD, M. L.

Options to Open-Field and Shielded Enclosure Electromagnetic Compatibility Measurements

Proc., 4th Symp. Electromagn. Compat., 10-12 March 1981, Zurich, Switzerland

March 1981

KANDA, M.

Theoretical and Experimental Investigations of Loading Effects Due to a Perfectly Conducting

Rectangular Cylinder in a Transverse Electromagnetic Cell

Proc., 4th Symp. Electromagn. Compat., 10-12 March 1981, Zurich, Switzerland

March 1981

LARSEN, E. B.; RIES, F. X.

Design and Calibration of the NBS Isotropic Electric-Field Monitor (EFM-5), 0.2 to $1000 \mathrm{MHz}$

NBS TN 1033

March 1981

MA, M. T.; WILSON, P. F.; CHANG, D. C.

Excitation of a TEM Cell by a Vertical Electric Hertzian Dipole

NBS TN 1037

March 1981 
CRAWFORD, M. L.

Evaluation of a Reverberation Chamber Facility for Performing EM Radiated Fields Susceptibility Measurements

NBSIR 81-1638

February 1981

BOWMAN, R. R.

A Temperature Probe for Radio-Frequency Heated Material

NBSIR 81-1634

January 1981

CRAWFORD, M. L.; WORKMAN, J. L.

Predicting Free-Space Radiated Emissions from Electronic Equipment Using TEM Cell and Open-Field Site Measurements

Record, 1980 Intl. Electromagn. Compat. Symp.

1980

HILL, D. A.; WAIT, J. R.

Electromagnetic Characteristics of a Coaxial Cable with Periodic Slots

IEEE Trans. Electromagn. Compat., Vol. EMC-22, No. 4, pp. 303-307

November 1980

MILLER, C. K. S.

Challenges of EMI Measurements

Proc., Natl. Conf. of Standards Laboratories, 22-25 September 1980, pp. 8-1 through 8-17

September 1980

CRAWFORD, M. L.; WORKMAN, J. L.

Spherical Dipole for Radiating Standard Fields

Dig., 1980 Conf. on Prec. Electromagn. Meas., 23-27 June 1980, Braunschweig, FDR, pp. 424429

June 1980

KANDA, M.

The Characteristics of a Linear Antenna with Tapered Resistive and Capacitive Loading

Proc., IEEE Antenna Propagat. Soc. Symp., 2-6 June 1980, Quebec, Canada, Vol. 2, AP. 18-2, pp. 696-699

June 1980

KANDA, M.

The Time-Domain Characteristics of a Traveling-Wave Linear Antenna with Linear and Nonlinear

Parallel Loads

IEEE Trans. Antennas Propagat., Vol. AP-28, No. 2, pp. 267-276 March 1980

SREENIVASIAH, I.; CHANG, D. C.; MA, M. T.

Characterization of Electrically Small Radiating Sources by Tests Inside a Transmission Line Cell NBS TN 1017

March 1980

HILL, D. A.; WAIT, J. R.

Propagation Along a Coaxial Cable with a Helical Shield

IEEE Trans. Microwave Theory Tech., Vol. MTT-28, No. 2, pp. 84-89

February 1980 
KANDA, M.

Transients in Resistively Loaded Antennas and Their Comparison with Conical Antennas and a TEM Horn

IEEE Trans. Antennas Propagat., Vol. AP-28, No. 1, pp. 132-136 January 1980

KANDA, M.

Analytical and Numerical Techniques for Analyzing an Electrically Short Dipole with a Nonlinear Load

IEEE Trans. Antennas Propagat., Vol. AP-28, No. 1, pp. 71-78 January 1980

KANDA, M.; RIES, F. X.; BELSHER, D. R.

A Broadband Isotropic, Real-Time, Electric Field Sensor (BIRES) Using Resistively Loaded Dipoles NBSIR 79-1622

December 1979

KANDA, M.

The Effects of Resistive Loading on TEM Horns

NBSIR 79-1601

August 1979

ARTHUR, M. G.

Impulse Spectral Intensity -- What is it?

Proc., 1978 Electromagn. Interference Workshop NBS SP 551

July 1979

TIPPET, J. C.; CHANG, D. C.

Dispersion and Attenuation Characteristics of Modes in a TEM Cell with a Lossy Dielectric Slab NBSIR 79-1615

July 1979

CRAWFORD, M.L.

Comparison and Selection of Techniques for Measuring EM Radiated Emissions and Susceptibility of Large Equipment

Proc., 1979 Symp. and Technical Exhib. on Electromagn. Compat. (3rd), 1-3 May 1979, Rotterdam, Netherlands, pp. 115-122

May 1979

MA, M. T.

A Theoretical Study of Unbalanced Ground Effects on Receiving Dipoles

NBSIR 79-1605

May 1979

SREENIVASIAH, I.; CHANG, D. C.

Variational Expression for the Scattering Matrix of a Coaxial Line Step Discontinuity and its Application to an Over Moded Coaxial TEM Cell

NBSIR 79-1606

May 1979

CRAWFORD, M. L.; WORKMAN, J. L.

Using a TEM Cell for EMC Measurements of Electronic Equipment NBS TN 1013

April 1979 
DECKER, W. F.; CRAWFORD, M. L.; WILSON, W. A.

Construction of a Large Transverse Electromagnetic Cell

NBS TN 1011

February 1979

KANDA, M.

The Time Domain Characteristics of a Traveling Wave Linear Antenna with Linear and Non-Linear Loads

NBSIR 78-892 (Restricted)

February 1979

KANDA, M.

The Characteristics of a Traveling-Wave, Linear Antenna with a Nonlinear Load

Dig. Abstract, 1979 IEEE Antenna Propagat. Soc. Symp.

January 1979

MILLER, C. K. S.

EMI - A Problem of Growing Concern

Report of the 63rd Natl. Conf. on Weights and Measures NBS SP 532

1978

KANDA, M.

Analytical and Numerical Techniques for Analyzing an Electrically Short Dipole with a Nonlinear Load NBSIR 78-898

November 1978

LAWTON, R. A.; ONDREJKA, A. R.

Antennas and the Associated Time Domain Range for the Measurement of Impulsive Fields NBS TN 1008

November 1978

TIPPET, J. C.; CHANG, D. C.

Characteristic Impedance of a Rectangular Coaxial Line With Offset Inner Conductor

IEEE Trans. Microwave Theory Technol., Vol. MTT-26, No. 11, pp. 876-883

November 1978

CRAWFORD, M. L.; WORKMAN, J. L.; THOMAS, C. L.

Expanding the Bandwidth of TEM Cells for EMC Measurements

IEEE Trans. Electromagn. Compat., Vol. EMC-20, No. 3, pp. 368-375

August 1978

ANDREWS, J. R.; BALDWIN, E. E.

SHF Impulse Generator

NBSIR 78-888

June 1978

CRAWFORD, M. L.; WORKMAN, J. L.

Asymmetric Versus Symmetric TEM Cells for EMI Measurements

Proc., 1978 IEEE Intl. Symp. on Electromagn. Compat., 20-22 June 1978, Atlanta, GA, pp. 204-210

June 1978 
ANDREWS, J. R.; BALDWIN, E. E.

UHF Impulse Generator

NBSIR 78-880

April 1978

KANDA, $M$.

Transients in Resistively Loaded Antennas and Their Comparison with Conical Antennas and TEM

Horns

NBSIR 78-876

March 1978

TIPPET, J. C.; CHANG, D. C.

Higher Order Modes in Rectangular Coaxial Line with Infinitely Thin Inner Conductor

NBSIR 78-873

March 1978

ADAMS, J. W.; TAGGART, H. E.

Electromagnetic Compatibility Evaluation of Seven Electronic Brake Systems

NBSIR 78-872

February 1978

FLANIGAN, W. F. Jr.; BOWMAN, R. R.; LOWELL, W. R.

Nonmetallic Electrode System for Recording EEG and ECG in Electromagnetic Fields

Physiology and Behavior, Vol. 18, No. 3, pp. 531-533

1977

KANDA, M.

A Relatively Short Cylindrical Broadband Antenna with Tapered Resistive Loading for Picosecond Pulse Measurements

IEEE Trans. Antennas Propagat., Vol. AP-26, No. 3, pp. 439-447

1977

LARSEN, E. B.; SHAFER, J. F.

Surveys of Electromagnetic Field Intensities Near Representative Higher-Power FAA Transmitting Antennas

FAA-RD-77

December 1977

KANDA, M.

The Characteristics of Broadband, Isotropic Electrical Field and Magnetic Field Probes NBSIR 77-868

November 1977

ANDREWS, J. R.; ARTHUR, M. G.

Spectrum Amplitude--Definition, Generation and Measurement

NBS TN 699

October 1977

CRAWFORD, M. L.; WORKMAN, J. L.; THOMAS, C. L.

Generation of EM Susceptibility Test Fields Using a Large Absorber-Loaded TEM Cell

IEEE Trans. Instrum. Meas., Vol. IM-26, No. 3, pp. 336-343

September 1977 
ADAMS, J. W.; KANDA, M.; SHAFER, J. F.; WU, Y.

Near-Field Electric Field Strength Levels of EM Environments Applicable to Automotive Systems Proc., 1977 IEEE Intl. Symp. on Electromagn. Compat., 2-4 August 1977, Seattle, WA, pp. 336-343

August 1977

CRAWFORD, M. L.; THOMAS, C. L.

Converting a Rectangular Shielded Enclosure into a TEM Transmission Cell for EMI Measurements

Proc., 1977 IEEE Intl. Symp. on Electromagn. Compat., 2-4 August 1977, Seattle, WA, pp. 1-6 August 1977

KANDA, M.

A Relatively Short Cylindrical Broadband Antenna with Tapered Resistive Loading for Picosecond Pulse Measurements

NBSIR 77-861

August 1977

KANDA, M.

A Broadband Antenna with Tapered Resistive Loading for EMI Measurements

Proc., 1977 IEEE Intl. Symp. on Electromagn. Compat., 2-4 August 1977, Seattle, WA, pp. 13-18

August 1977

BENSEMA, W. D.

Amplitude, Time, and Frequency Statistics of Quasi-Impulsive Noise Proc., 1977 Electromagn. Compat. Symp., 18-30 June 1977, Montreux, Switzerland, pp. 347-352

June 1977

KANDA, $M$.

The Characteristics of a Relatively Short Broadband Linear Antenna with Tapered Resistive Loading Proc., Intl. Symp. Antenna Propagat. Soc., 20-22 June 1977, pp. 230-233 June 1977

BENSEMA, W. D.

A Noise Spectrum Measurement System Using the Fast Fourier Transform IEEE Trans. Electromagn. Compat., Vol. EMC-19, No. 2, pp. 37-43 May 1977

ANDREWS, J. R.

Impulse Generator Spectrum Amplitude Measurement Techniques

IEEE Trans. Instrum. Meas., Vol. IM-25, No. 4, pp. 380-384

December 1976

GREENE, F. M.

Measurement of RF Power-Absorption in Biological Specimens (10 to $100 \mathrm{MHz}$ )

NBS TN 687

November 1976

LARSEN, E. B.; ANDREWS, J. R.; BALDWIN, E. E.

Sensitive Isotropic Antenna with Fiber-Optic Link to a Conventional Receiver NBSIR 75-819

September 1976 
TIPPET, J. C.; CHANG, D. C.

A New Approximation for the Capacitance of a Rectangular, Coaxial, Strip Transmission Line IEEE Trans. Microwave Theory Tech., pp. 602-604

September 1976

CRAWFORD, M. L.

Improved Techniques and Instrumentation for EMC Measurements

IEEE 1976 Intl. Symp. on Electromagn. Compat., 13-15 July 1976, Washington, DC

July 1976

LARSEN, E. B.; ANDREWS, J. R.

Using Fiber Optics in A Broadband, Sensitive, Isotropic Antenna - $15 \mathrm{KHz}$ to $150 \mathrm{MHz}$

Proc., 1976 IEEE Intl. Symp. on Electromagn. Compat., 13-15 July 1976, Washington, DC July 1976

CRAWFORD, M. L.

Experimental Evaluation of the Radiation Characteristics of Dipole Sources Enclosed in a TEM

Transmission Cell

Proc., 1976 Conf. on Precision Electromagn. Meas., 28 June-1 July 1976, Boulder, CO, pp.

$57-59$

June 1976

REEVE, G. R.

A Standard Percent Modulation Measurement System

Dig. 1976 Conf. on Prec. Electromagn. Meas., 28 June-1 July 1976, Boulder, CO, pp. 165-166 June 1976

TIPPET, J. C.; CHANG, D. C.; CRAWFORD, M. L.

An Analytical and Experimental Determination of the Cutoff Frequencies of Higher-Order TE Modes in a TEM Cell

NBSIR 76-841

June 1976

BOWMAN, R. R.

Calibration Techniques for Electromagnetic Hazard Meters: $500 \mathrm{MHz}$ To $20 \mathrm{GHz}$

NBSIR 75-805

April 1976

ADAMS, J. W.; CRAWFORD, M. L.; SHAFER, J. F.

Electromagnetic Interference (EMI) Measurements for Automotive Applications

Proc., 1976 SAE Automotive Congress Conf., 23-27 February 1976, Detroit, MI, pp. 1-6

February 1976

BOWMAN, R. R.

A Probe for Measuring Temperature in Radio-Frequency-Heated Material

IEEE Trans. Microwave Theory Technol., Vol. MTT-24, No. 1, pp. 43-45

January 1976

TIPPET, J. C.; CHANG, D. C.

Radiation Characteristics of Dipole Sources Located Inside a Rectangular, Coaxial Transmission Line NBSIR 75-829

January 1976 
FITZGERRELL, R. G.

Experimental RF Field Polarization Meter

FAA-RD-74-188

1975

CRAWFORD, M. L.

Techniques for Measurement of Electromagnetic Radiation and Susceptibility of Electronic Equipment Proc., 1975 Symp. and Technical Exhib. on Electromagn. Compat. (1st), 20-22 May 1975, Montreux, Switzerland, pp. 38-44

May 1975

KANDA, M.; ADAMS, J. W.

Amplitude Statistics of Electromagnetic Noise in Coal Mines

Dig., Intl. Union of Radio Sci. (Union Radio Scientifique Internationale), IEEE Trans. Antennas Propagat.

May 1975

BELSHER, D. R.

Development of Near-Field Electric Energy Density Meter Model EDM-2

Department of Health, Education, and Welfare, Publ. No. (NIOSH) 75-140

March 1975

CRAWFORD, M. L.

Generation of Standard EM Fields for Calibration of Power Density Meters $20 \mathrm{kHz}$ to $1000 \mathrm{MHz}$

NBSIR 75-804

January 1975

GREENE, F. M.

Development of Electric and Magnetic Near-Field Probes

NBS TN 658

January 1975

ANDREWS, J. R.

Picosecond Pulse Generators Using Microminiature Mercury Switches

NBSIR 74-377

1974

SCOTT, W. W., Jr.; ADAMS, J. W.; BENSEMA, W. D.; DOBROSKI, H.

Electromagnetic Noise in Lucky Friday Mine

NBSIR 74-391

1974

CRAWFORD, M. L.

Generation of Standard EM Fields Using TEM Transmission Cells

IEEE Trans. Electromagn. Compat., Vol. EMC-16, No. 4, pp. 189-195 November 1974

KANDA, $M$.

Time and Amplitude Statistics for Electromagnetic Noise in Mines

NBSIR 74-378

July 1974 
NEWELL, A. C.; CRAWFORD, M. L.

Planar Near-Field Measurements on High Performance Array Antennas NBSIR 74-380

July 1974

ADAMS, J. W.; BENSEMA, W. D.; TOMOEDA, N. C.

Surface Magnetic Field Noise Measurements at Geneva Mine

NBSIR 74-369

June 1974

ADAMS, J. W.; BENSEMA, W. D.; KANDA, M.

Electromagnetic Noise in Grace Mine

NBSIR 74-388

June 1974

BENSEMA, W. D.; KANDA, M.; ADAMS, J. W.

Electromagnetic Noise in Itmann Mine

NBSIR 74-390

June 1974

CRAWFORD, M. L.

Evaluation of Reflectivity Level of Anechoic Chambers Using Isotropic, 3-Dimensional Probing

Proc., 1974 Intl. IEEE Antenna Propagat. Soc. Symp., 10-12 June 1974, Atlanta, GA, pp. 28-34 June 1974

HUDSON, P. A.; MELQUIST, D. G.; ONDREJKA, A. R.; WERNER, P. E.

Completion of the Program to Evaluate/Improve Instrumentation and Test Methods for Electroexplosive Device Safety Qualification

NBSIR 74-379

June 1974

KANDA, M.; ADAMS, J. W.; BENSEMA, W. D.

Electromagnetic Noise in McElroy Mine

NBSIR 74-389

June 1974

SIMPSON, P. A.

Broadband Pulsed/CW Calibration Signal Standard for Field Intensity Meter (FIM) Receivers

NBSIR 74-371

June 1974

ARTHUR, M. G.

Impulse Spectral Intensity -- What is it?

NBSIR 74-365

May 1974

GREENE, F. M.

Development and Construction of an Electromagnetic Near-Field Synthesizer

NBS TN 652

May 1974 
BENSEMA, W. D.; KANDA, M.; ADAMS, J. W.

Electromagnetic Noise in Robena No. 4 Coal Mine

NBS TN 654

April 1974

REEVE, G. R.

Calibration of Impulse Noise Generators

NBSIR 73-343

1973

BAIRD, R. C.

Methods of Calibrating Microwave Hazard Meters

Proc., 1973 Intl. Symp on Biological Effects and Health Hazards of Microwave Radiation, 15-18

October 1973, Warsaw, Poland,pp. 228-236

October 1973

BOWMAN, R. R.

Some Recent Developments in the Characterization and Measurement of Hazardous Electromagnetic Fields

Proc., 1973 Intl. Symp. on Biological Effects and Health Hazards of Microwave Radiation, 15-18 October 1973, Warsaw, Poland, pp. 217-227

October 1973

LARSEN, E. B.

Calibration of Radio Receivers to Measure Broadband Interference

NBSIR 73-335

September 1973

KANDA, M.; ADAMS, J. W.

Amplitude Statistics of Electromagnetic Noise in Coal Mines

Proc., 1973 Thru-the-Earth Electromagn. Workshop, 15-17 August 1973, Colorado School of Mines, Golden, CO, pp. 156-160

August 1973

HUDSON, P. A.; MELQUIST, D. G.; ONDREJKA, A. R.; WERNER, P. E.

A Program to Evaluate/Improve Instrumentation and Test Methods for Electroexplosive Device Safety Qualification

NBSIR 73-323

June 1973

NAHMAN, N.S.

Miniature Superconductive Coaxial Transmission Lines

Proc., IEEE, Vol. 61, pp. 76-79

January 1973

BENSEMA, W. D.

Coal Mine Magnetic-Field Noise Measurements

Proc., 1972 W. Virginia Conf. on Coal Mine Electrotechnology (1st), 2-4 August 1972, West Virginia Univ., Morgantown, WV, pp. XII-1 through XII-12

August 1972 
ADAMS, J. W.; TAGGART, H. E.; SPAULDING, A. D.

Survey Report of the U.S. Bureau of Mines Electromagnetic Noise Measurement Program NBS Rept. 10723

November 1971

WACKER, P. F.; BOWMAN, R. R.

Quantifying Hazardous Electromagnetic Fields: Scientific Basis and Practical Considerations

IEEE Trans. Microwave Theory Tech., Vol. MTT-19, No. 22

February 1971

BOWMAN, R. R.

Quantifying Hazardous Electromagnetic Microwave Fields: Practical Considerations

Proc., 1969 Symp. Biological Effects and Health Implications of Microwave Radiation, 17-19

September 1969, Richmond, VA., BRH/DBE 70-2, U.S. Dept. of Health, Education, and

Welfare, Rockville, MD, pp. 204-209

June 1970

WACKER, P. F.

Quantifying Hazardous Microwave Fields: Analysis

Proc., 1969 Symp. on Biological Effects and Health Implications of Microwave Radiation, 17-19 September 1969, Richmond, VA, BRH/DBE 70-2, U.S. Dept. of Health Education, and

Welfare, Rockville, MD pp. 197-203 June 1970

\section{LAWTON, R. A.}

A New Standard for Electric Field Strength

IEEE Trans. Instrum. Meas., Vol. IM-19, pp. 45-51

February 1970 


\section{MICROWAVE METROLOGY}

JARGON, J. A.

Revised Uncertainty of the NIST $30 \mathrm{MHz}$ Phase Shifter Measurement Service Proc., Meas. Sci. Conf., 5-6 February 1998, Pasadena, CA ( pages not numbered) February 1998

DEGROOT, D. C.; MARKS, R. B.; JARGON, J. A.

A Method for Comparing Vector Network Analyzers

Dig., ARFTG Conf., 4-5 December 1997, Portland, OR, pp. 107-114

December 1997

DEGROOT, D. C.; JARGON, J. A.

RF and Microwave Device Measurements Using a Digital Sampling Oscilloscope

Instrum. Newsletter, Vol. 9, No. 4, pp. T1-4

December 1997

MARKS, R. B.; HEUTMAKER, M. S.

The 1997 Wireless Communications Conference

NIST JRES, Vol. 102, No. 6

November/December 1997

MARKS, R. B.

Formulations of the Basic Vector Network Analyzer Error Model Including Switch Terms

Dig., ARFTG Conf., 4-5 December 1997, Portland, OR, pp. 115-126

December 1997

MARKS, R. B.; ALLEY, G. D.

The Automatic Radio Frequency Techniques Group Conference on "Characterization of Broadband Telecommunications Components and Systems"

NIST JRES, Vol. 102, No. 6

November/December 1997

WILLIAMS, D. F.; WALKER, D. K.

Series-Resistor Calibration

Dig., ARFTG Conf., 4-5 December 1997, Portland, OR, pp. 131-137

December 1997

JUROSHEK, J. R.

A Direct Calibration Method for Measuring Equivalent Source Mismatch

Microwave J., pp. 106-118

October 1997

WILLIAMS, D. F.; JANEZIC, M.D.; RALSTON, A.

Quasi-TEM Model for Coplanar Waveguide on Silicon

Dig., Electrical Performance of Electronic Packaging Conf., 27-29 October 1997, San Jose, CA, pp. $225-228$

October 1997

RANDA, J.; TERRELL, L. A.

Noise-Temperature Measurement System for the WR-28 Band

NIST TN 1395

August 1997 
WILLIAMS, D. F.; MARKS, R. B.; HAYDEN, L. A.

A Complete Multinode Equivalent-circuit Theory for Electrical Design

NIST JRES, Vol. 102, No. 4, pp. 405-423

July/August 1997

STORK, F.J.B.; BEALL, J. A.; ROSHKO, A.; DEGROOT, D. C.; RUDMAN, D. A.; ONO, R. H. Microwave Characterization of Coplanar Waveguide Transmission Lines Fabricated by Ion Implantation Patterning of $\mathrm{YBa}_{2} \mathrm{Cu}_{3} \mathrm{O}_{7-\delta}$

IEEE Trans. Appl. Supercond., Vol. 7, No. 2, pp. 2780-2783

June 1997

WILLIAMS, D. F.

Characterization of Embedded Multiconductor Transmission Lines

Dig., IEEE 1997 MTT Intl. Symp., 8-12 June 1997, Denver, CO, pp. 1773-1776

June 1997

GINLEY, R. A.

Line-Reflect-Match (LRM) Calibration Technique for the Dual Six-Port ANA

IEEE Trans. Instrum. Meas., Vol. 46, No. 2, pp. 523-526

April 1997

WALKER, D. K.; WILLIAMS, D. F.

Compensation for Geometrical Variations in Coplanar Waveguide Probe-Tip Calibration

IEEE Microwave Guided Wave Lett., Vol. 7, No. 4, pp. 97-99

April 1997

MARKS, R. B.; JARGON, J. A.; JUROSHEK, J. R.

Calibration Comparison Method for Vector Network Analyzers

Dig., 48th Auto. RF Tech. Group Conf., 5-6 December 1996, Clearwater, FL, pp. 38-45

December 1996

WILLIAMS, D. F.

Calibration in Multiconductor Transmission Lines

Dig., 48th Auto. RF Tech. Group Conf., 5-6 December 1996, Clearwater, FL, pp. 46-53

December 1996

WILLIAMS, D. F.; OLYSLAGER, F.

Modal Cross Power in Quasi-TEM Transmission Lines

IEEE Microwave Guided Wave Lett., Vol 6., No. 11, pp. 413-415

November 1996

DEGROOT, D. C.; WALKER, D. K.; MARKS, R. B.

Impedance Mismatch Effects on Propagation Constant Measurements

Proc., 5th Topical Mtg. Elect. Perf. of Electronic Packaging, 28-30 October 1996, Napa, CA, pp. 141-143

October 1996

DEGROOT, D. C.; RUDMAN, D. A.; ZANG, K.; MA, Q. V.; KATO, H.; JAEGER, N. A. F.

Planar Microwave Devices Fabricated by Ion-Implantation Patterning of High-Temperature

Superconductors

Appl. Phys. Lett., Vol. 69, No. 14, pp. 2119-2121

September 1996 
MARKS, R. B.; WILLIAMS, D. F.

Electrical Characterization Methods for High-Speed Interconnects

Intl. J. Microcircuits and Electronic Packaging, Vol. 18, No. 3, pp. 207-216

August 1996

MASTERSON, K. D.; NOVOTNY, D. R.; CAVCEY, K. H.

Standard Antennas Designed with Electrooptic Modulators and Optical-Fiber Linkage

Proc., SPIE Intense Microwave Pulses IV Conf., 4-9 August 1996, Denver, CO, Vol. 2843, pp. 188-196

August 1996

WILLIAMS, D. F.

Thermal Noise in Lossy Waveguides

IEEE Trans. Microwave Theory Tech., Vol. 44, No. 7, pp. 1067-1073

July 1996

GINLEY, R. A.

Calibrating a Dual Six Port ANA With a Single Impedance Standard

Proc., 1996 Conf. on Prec. Electromagn. Meas., 17-20 June 1996, Braunschweig, Germany, pp. 532-533

June 1996

HUANG, D. X.; REBULDELA, G.; HARPER, J.

RF-DC Differences of Micropotentiometers

Proc., 1996 Conf. on Prec. Electromagn. Meas., 17-20 June 1996, Braunschweig, Germany, pp. $460-461$

June 1996

WILLIAMS, D. F.; BELQUIN, J.; DAMBRINE, G.; FENTON, R.

On-Wafer Measurement at Millimeter Wave Frequencies

Proc., 1996 IEEE MTT Intl. Microwave Symp., 17-21 June 1996, San Francisco, CA, pp. 16831686

June 1996

WEIDMAN, M. P.

Direct Comparison Transfer of Microwave Power Sensor Calibrations

NIST TN 1379

January 1996

JARGON, J. A.; MARKS, R. B.

Two-Tier Multiline TRL for Calibration of Low-Cost Network Analyzers

Dig., 46th Auto. RF Tech. Group Conf. 30 Nov.-1 Dec. 1995, Scottsdale, AZ, pp. 1-8 December 1995

WILLIAMS, D. F.; SCHAPPACHER, J. B.

Line-Reflect-Match Calibrations with Nonideal Microstrip Standards

Dig., 46th Auto. RF Tech. Group Conf., 30 Nov.-1 Dec. 1995, Scottsdale, AZ, pp. 35-38

December 1995

JARGON, J. A.; MARKS, R. B.; WILLIAMS, D. F.

Coaxial Line-Reflect-Match Calibration

Proc., Asia-Pacific Microwave Conf., 10-13 October 1995, Taejon, Korea, Vol. 1, pp. 86-89

October 1995 
JARGON, J. A.

A $30 \mathrm{MHz}$ Comparison Receiver

Proc., Asia-Pacific Microwave Conf., 10-13 October 1995, Taejon, Korea, Vol. 1, pp. 94-96 October 1995

MARKS, R. B.; JARGON, J. A.; PAO, C. K.; WEN, C. P.

Electrical Measurements of Microwave Flip-Chip Interconnections

Proc., Int1. Symp on Microelectronics, 24-26 October 1995, Los Angeles, CA, pp. 424-429 October 1995

\section{CLAGUE, F. R.}

A Calibration Service for Coaxial Reference Standards for Microwave Power

NIST TN 1374

May 1995

MARKS, R. B.; JARGON, J. A.; PAO, C. K.; WEN, C. P.; SHIH, Y. C.

Microwave Characterization of Flip-Chip MMIC Components

Proc., 1995 Electronic Components and Tech. Conf. 21-24 May 1995, Las Vegas, NV, pp. 343-350

May 1995

MARKS, R. B.; JARGON, J. A.; PAO, C. K.; WEN, C. P.

Microwave Characterization of Flip-Chip MMIC Interconnections

Proc., 1995 IEEE MMT-S Intl. Microwave Symp., 15-16 May 1995, Orlando, FL,

pp. 1463-1466

May 1995

PUCIC, S. P.; DAYWITT, W. C.

Single-Port Technique for Adaptor Efficiency Evaluation

Proc., Auto. RF Tech. Group Conf., 15 May 1995, Orlando, FL, pp. 113-118 May 1995

MARKS, R. B.; WILLIAMS, D. F.

Comments on "Conversation Between S, Z, Y, h, ABCD, and T Parameters Which are Valid for Complex

Source and Load Impedances"

IEEE Trans. Microwave Theory Tech., Vol. 43, No. 4, pp. 914-915

April 1995

WILLIAMS, D. F.; MARKS, R. B.

LRM Probe-Tip Calibrations Using Nonideal Standards

IEEE Trans. Microwave Theory Tech., Vol. 43, No. 2, pp. 466-469

February 1995

WILLIAMS, D. F.; MARKS, R. B.

Compensation for Substrate Permittivity in Probe-Tip Calibration

Proc., 44th Auto. RF Tech. Group Conf., 1-2 December 1994, Boulder, CO, pp. 20-30

December 1994

MARKS, R. B; WILLIAMS, D. F.

Accurate Electrical Characterization of High-Speed Interconnections

Proc., Intl. Symp on Microelectronics, 14-17 November 1994, Boston, MA, pp. 96-101

November 1994 
CLAGUE, F. R.; SPLETT, J. D.

Developing a NIST Coaxial Microwave Power Standard at $1 \mathrm{~mW}$

Proc., NCSL Conf., 3 August 1994, Chicago, IL, pp. 291-298

August 1994

CLAGUE, F. R.

A Method to Determine the Calorimetric Equivalence Correction for a Coaxial Microwave Microcalorimeter

IEEE Trans. Instrumen.Meas., Vol. 43, No. 3, pp. 421-425

June 1994

MARKS, R. B.; WILLIAMS, D. F.

Microwave Characterization of Printed Circuit Transmission Lines

Proc., Natl. Electronic Packaging and Production Conf., June 16, 1994, Boston, MA, pp. 520-527

June 1994

WILLIAMS, D. F.; MARKS, R. B.

On-Wafer Impedance Measurement on Lossy Substrates

IEEE Microwave Guided Wave Lett., Vol. 4, No. 6, pp. 175-176

June 1994

ENGEN, G. F.; JUDISH, R. M.; JUROSHEK, J. R.

The Multi-State Two-port: An Alternative Transfer Standard

Dig, 43rd Auto. RF Tech. Group Conf., 27 May 1994, San Diego, CA, pp. 11-18

May 1994

JUROSHEK, J. R.; FREE, G. M.

Measurements of the Characteristic Impedance of Coaxial Air Line Standards

IEEE Trans. Microwave Theory Tech., Vol. 42, No. 2, pp. 186-19l

February 1994

JARGON, J. A.; GINLEY, R. A.; SUTTON, D. D.

The NIST $30 \mathrm{MHz}$ Linear Measurement System

NIST JRES, Vol. 99, No. 1, pp. 19-30

January-February 1994

JARGON, J. A.

A Revised Uncertainty Analysis for the NIST 30-MHz Attenuation Calibration System

1994 Meas. Sci. Conf., 27-28 January 1994, Pasadena, CA (no page numbers)

January 1994

CLAGUE, F. R.

NIST Model PM2 Power Measurement System for $1 \mathrm{~mW}$ at $1 \mathrm{GHz}$

NISTIR 5016

December 1993

MARKS, R. B.; WILLIAMS, D. F.

Verification of Commercial Probe-Tip Calibrations

Dig., 42nd Auto. RF Tech. Group Conf., 2-3 December 1993, San Jose, CA, pp. 37-41

December 1993 
WILLIAMS, D. F.; MARKS, R. B.

LRM Probe-Tip Calibrations with Imperfect Resistors and Loss Lines

Dig., 42nd Auto. RF Tech. Group Conf., 2-3 December 1993, San Jose, CA, pp. $32-36$

December 1993

CLAGUE, F. R.

Microcalorimeter for $7 \mathrm{~mm}$ Coaxial Transmission Line

NIST TN 1358

August 1993

WILLIAMS, D. F.; MARKS, R. B.

Accurate Transmission Line Characterization

IEEE Microwave Guided Wave Lett., Vol. 3, No. 8, pp. 247-249

August 1993

WILLIAMS, D. F.; MARKS, R. B.

Reciprocity Relations in Waveguide Junctions

IEEE Trans. Microwave Theory Tech., Vol. 41, No. 6/7, pp. 1105-1110

June/July 1993

CLAGUE, F. R.; VORIS, P. C.

Coaxial Reference Standard for Microwave Power

NIST TN 1357

April 1993

MARKS, R. B.

Comments on "Protecting EFIE-Based Scattering Computations from Effects of Interior Resonances"

IEEE Trans. Antennas Propagat., Vol. 41, No. 3, pp. 387-389

March 1993

JARGON, J. A.; REBULDELA, G.

Measurement Service for High-Power CW Wattmeters at the National Institute of Standards and Technology

Proc., Meas. Sci. Conf., 20-22 January 1993, Anaheim, CA, Session 5-B (no page numbers) January 1993

SHERWOOD, G. V.

Dimensional Characterization of Precision Coaxial Transmission Line Standards

Proc., Meas. Sci. Conf., 20-22 January 1993, Anaheim, CA, pp. 1-10

January 1993

MARKS, R. B.; WILLIAMS, D. F.

Interconnection Transmission Line Parameter Characterization

Dig., 40th Auto. RF Tech. Group Conf., 3-4 December 1992, Orlando, FL, pp. 88-95

December 1992

WALKER, D. K.; WILLIAMS, D. F.; MORGAN, J. M.

Planar Resistors for Probe Station Calibration

Dig., 40th Auto. RF Tech. Group Conf., 3-4 December 1992, Orlando, FL, pp. 1-9

December 1992 
WILLIAMS, D. F.; MARKS, R. B.

Calibrating On-Wafer Probes to the Probe Tips

Dig., 40th Auto. RF Tech. Group Conf., 3-4 December 1992, Orlando, FL, pp. 136-143

December 1992

REBULDELA, G.; JARGON, J. A.

High Power CW Wattmeter Calibration at NIST

NIST JRes, Vol. 97, No. 6, pp. 673-687

November-December 1992

WILLIAMS, D. F.; MARKS, R. B.; WALKER, D. K.; CLAGUE, F. R.

Wafer Probe Transducer Efficiency

IEEE Microwave and Guided Wave Lett., Vol. 2, No. 10, pp. 388-390

October 1992

MARKS, R. B.; WILLIAMS, D. F.

A General Waveguide Circuit Theory

NIST JRES, Vol. 97, No. 5, pp. 533-562

September-October 1992

HUANG, D. X.; REBULDELA, G.

High-Frequency Response of Micropotentiometers

Proc., NCSL, 2-6 August 1992, Washington, DC, pp. 547-555

August 1992

MARKS, R. B.; WILLIAMS, D. F.

Accurate Experimental Characterization of Interconnects: A Discussion of "Experimental Electrical Characterization of Interconnects and Discontinuities in High-Speed Digital Systems"

IEEE Trans. Components, Hybrids, and Manufacturing Technol., Vol. 15, No. 4, pp. 601-604 August 1992

WILLIAMS, D. F.; MARKS, R. B.

Comments on "Characterization of Resistive Transmission Lines by Short-Pulse Propagation"

IEEE Microwave Guided Wave Lett., Vol. 2, No. 8, p. 346

August 1992

CLAGUE, F. R.

A New Coaxial Microwave Microcalorimeter Evaluation Technique

Dig., 1992 Conf. on Prec. Electromagn. Meas., 9-12 June 1992, Paris, France, pp. 387-388 June 1992

MARKS, R. B.; WILLIAMS, D. F.

Traceability for On-Wafer MMIC Measurements

Dig., 1992 Conf. on Prec. Electromagn. Meas., 9-12 June 1992, Paris, France, pp. 91-92 June 1992

REBULDELA, G.; JARGON, J. A.

High Power CW Wattmeter Calibration at NIST

Dig., 1992 Conf. on Prec. Electromagn. Meas., 9-12 June 1992, Paris, France, pp. 381-382 June 1992 
WILLIAMS, D. F.; MARKS, R. B.

Verification of Scattering Parameter Measurements

Dig., 1992 Conf. on Prec. Electromagn. Meas., 9-12 June 1992, Paris, France, pp. 371-372 June 1992

ESTIN, A. J.; JUROSHEK, J. R.; MARKS, R. B.; CLAGUE, F. R.; ALLEN, J. W.

Basic RF and Microwave Measurements: A Review of Selected Programs

Metrologia, Vol. 29, No. 2, pp. 135-151

May 1992

MARKS, R. B.

Comments on "Rapid Pulsed Microwave Propagation"

IEEE Microwave Guided Wave Lett., Vol. 2, No. 5, p. 204

May 1992

\section{WILLIAMS, D. F.; MARKS, R. B.}

Frequency-Dependent Transmission Line Parameters

Dig., IEEE Topical Meeting on Electrical Performance of Electronic Packaging, 22-24 April

1992, Tucson, AZ, pp. 125-127

April 1992

FREE, G. M.; JONES, R. N.

Calibration Service for Low-Loss, Three-Terminal Capacitance Standards at $100 \mathrm{kHz}$ and $1 \mathrm{MHz}$

NIST TN 1348

February 1992

FURLOW, R.; SHIMODA, R. Y.; WILLIAMS, D. F.; MARKS, R. B.; GUPTA, K. C.

Benchmark for the Verification of Microwave CAD Software

Proc., 38th Auto. RF Tech. Group Conf., 5-6 December 1991, San Diego, CA, pp. 97-106

December 1991

MARKS, R. B.; WILLIAMS, D. F.

Reciprocity Relations for On-Wafer Power Measurements

Proc., 38th Auto. RF Tech. Group Conf., 5-6 December 1991, San Diego, CA,pp. 82-89

December 1991

WILLIAMS, D. F.; MARKS, R. B.; DAVIDSON, A.

Comparison of On-Wafer Calibrations

Proc., 38th Auto. RF Tech. Group Conf., 5-6 December 1991, San Diego, CA, pp. 68-81

December 1991

MARKS, R. B.; WILLIAMS, D. F.

Transmission Line Capacitance Measurement

IEEE Microwave Guided Wave Lett., Vol. 1, No. 9, pp. 243-245

September 1991

REEVE, G. R.

Millimeter Wave Metrology at the National Institute of Standards and Technology

Proc., 1991 NCSL Workshop and Symp., 18-22 August 1991, Albuquerque, NM, pp. 183-195 August 1991 
MLARKS, R. B.

A Multi-Line Method of Network Analyzer Calibration

IEEE Trans. Microwave Theory Tech., Vol. MTT-39, No. 7, p. 1205-1215

July 1991

MLARS, R. B.; WILLIAMS, D. F.

Characteristic Impedance Determination Using Propagation Constant Measurement

IEEE Microwave Guided Wave Lett., Vol. 1, No. 6, p. 141-143

June 1991

JUDISH, R. M.; BURNS, J. G.

Measurement Program Compares Automatic Vector Analyzers

Microwaves and RF, pp. 203-206

May 1991

WILLIAMS, D. F.; MARKS, R. B.; PHILLIPS, K. R.

Translate LRL and LRM Calibrations

Microwaves and RF, pp. 78-84

February 1991

SHERWOOD, G. V.

Air Gage Size Measurement of Microwave Standards

Proc., 1991 Measurement Science Conf. 31 Jan.-1 Feb. 1991, Anaheim, CA, pp. 1-10

January 1991

REEVE, G. R.; MARKS, R. B.; BLACKBURN, D.

Microwave Monolithic Integrated Circuit-Related Metrology at the National Institute of Standards and Technology

IEEE Trans. Instrum. Meas., Vol. IM-39, No. 6, pp. 958-961

December 1990

CLAGUE, F. R.

Power Measurement System for $1 \mathrm{~mW}$ at $1 \mathrm{GHz}$

NIST TN 1345

November 1990

MARKS, R. B.

Multi-Line Calibration for MMIC Measurement

Dig., 36th Auto. RF Tech. Group Conf., 29-30 November 1990, Monterey, CA, pp. 47-55

November 1990

PHILLIPS, K. R.; WILLIAMS, D. F.

MMIC Package Characterization with Active Loads

Dig., 36th Auto. RF Tech. Group Conf., 29-30 November 1990, Monterey, CA, pp. 64-72 November 1990

WILliaMS, D. F.; MARKS, R. B.; PHILLIPS, K. R.; MIERS, T.

Progress Toward MMIC On-Wafer Standards

Dig., 36th Auto. RF Tech. Group Conf., 29-30 November 1990, Monterey, CA, pp. 73-83

November 1990 
WILLIAMS, D. F.; MARKS, R. B.

The Interpretation and Use of S-Parameters in Lossy Lines

Dig., 36th Auto. RF Tech. Group Conf., 29-30 November 1990, Monterey, CA, pp. 84-89 November 1990

HOLT, D. R.

Determination of Scattering Parameters with Respect to the Characteristic Impedance of Precision Coaxial Air-Line Standards

Dig., Conf. on Prec. Electromagn. Meas., 11-14 June 1990, Ottawa, Canada, pp. 282-283 June 1990

\section{MARKS, R. B.}

Comments on "Improved Calibration and Measurement of the Scattering Parameters of Microwave Integrated Circuits"

IEEE Trans. Microwave Theory Tech., Vol. MTT-38, No. 4, p.453 April 1990

MARKS, R. B.; PHILLIPS, K. R.

Wafer-Level ANA Calibrations at NIST

Dig., 34th Auto. RF Tech. Group Conf., 30 November-1 December 1989, Ft. Lauderdale, FL, pp. 11-25

December 1989

\section{WILLIAMS, D. F.}

Development of On-Wafer Microwave Standards at NIST

Dig., 34th Auto. RF Tech. Group Conf., 30 November-1 December 1989, Ft. Lauderdale, FL, pp. $5-10$

December 1989

\section{ADAIR, R. T.; LIVINGSTON, E. M. \\ Coaxial "Intrinsic" Impedance Standards \\ NIST TN 1333 \\ October 1989}

JUROSHEK, J. R.; HOER, C. A.; KAISER, R. K. F.

Calibrating Network Analyzers with Imperfect Test Ports

IEEE Trans. Instrum. Meas., Vol. 38, No. 4, pp. 898-901

August 1989

HOER, C. A.

Systematic Errors in Power Measurements Made with a Dual Six Port ANA;

NIST TN 1332

July 1989

WEIL, C. M.; MARLER, F. E.; MAJOR, J. R.; WEIDMAN, M. P.; RUSSELL, D. H.

Dual Six-Port Reflectometer Systems Using Waveguide in the Frequency Range $18-50 \mathrm{GHz}$.

Dig., 33rd Auto. RF Tech. Group Conf., 15-16 June 1989, Long Beach, CA, pp. 76-87 June 1989

\section{HOLT, D. R.}

Scattering Parameters Representing Imperfections in Precision Coaxial Air Lines

NIST JRES, Vol. 94, No. 2, pp. 117-133

March-April 1989 
CLAGUE, F. R.

The NIST Automated Coaxial Microwave Power Standard

Proc., 1989 Measurement Science Conf., 26-27 January 1989, Anaheim, CA, pp. 1C-1 through $1 \mathrm{C}-14$ January 1989

LIVINGSTON, E. M.; ADAIR, R. T.

Performance Evaluation of Radiofrequency, Microwave and Millimeter Wave Power Meters NIST TN 1310

December 1988

COUNAS, G. J.; YATES, B. C.

Measurement of Adapter Loss, Mismatch, and Efficiency Using the Dual Six-Port

NBSIR 88-3095

July 1988

HOER, C. A.

An Equivalent Circuit for Imperfect Transmission Line Connectors

Dig., Conf. on Prec. Electromagn. Meas., 7-10 June 1988, Tsukuba Science City, Japan, pp. 264-265

June 1988

HOLT, D. R.

Determination of Scattering Parameters from Precision Coaxial Air-Line Standards

Dig., Conf. on Prec. Electromagn. Meas., 7-10 June 1988, Tsukuba Science City, Japan, p. 263

June 1988

ADAIR, R. T.; RUSSELL, D. H.

A Calibration Service for $30 \mathrm{MHz}$ Attenuation and Phase Shift

NBS SP 250-32

April 1988

LARSEN, N. T.

Microwave Power Standards at the National Bureau of Standards

1987 NCSL Workshop and Symp., 12-16 July 1987, Denver, CO, pp. 34-1 through 34-7 July 1987

ENGEN, G. F.

On-Line Accuracy Assessment for the Dual Six-Port ANA: Background and Theory

IEEE Trans. Instrum. Meas., Vol. IM-36, No. 2, pp. 501-506

June 1987

HOER, C. A.

Some Questions and Answers Concerning Air Lines as Impedance Standards

Dig., 29th Auto. RF Tech. Group Conf., 12-13 June 1987, Las Vegas, NV, pp. 161-173 June 1987

HOER, C. A.

On-Line Accuracy Assessment for the Dual Six-Port ANA; Treatment of Systematic Errors IEEE Trans. Instrum. Meas., Vol. IM-36, No. 2, pp. 514-519

June 1987 
JUDISH, R. M.; ENGEN, G. F.

On-Line Accuracy Assessment for the Dual Six-Port ANA: Statistical Methods for Random Errors IEEE Trans. Instrum. Meas., Vol. IM-36, No. 2, pp. 507-513 June 1987

JUROSHEK, J. R.

On-Line Accuracy Assessment for the Dual Six-Port ANA: Experimental Results IEEE Trans. Instrum. Meas., Vol. IM-36, No. 2, pp. 520-523 June 1987

JUROSHEK, J. R.

A Study of Measurement of Connector Repeatability Using Highly Reflecting Loads

IEEE Trans. Microwave Theory Tech., Vol. MTT-35, No. 4, pp. 457-460 April 1987

ADAIR, R. T.; REEVE, G. R.; GATTERER, L. E.

The Expanding Need for Microwave and Millimeter Wave Calibration Services NCSL Newsletter, Vol. 27, No. 1, pp. 21-31 January 1987

SAULSBERY, L. F; ADAIR, R. T.

ANA Measurement Results on the Auto. RF Tech. Group Traveling Experiment Conf. Dig., 28th Auto. RF Tech. Group Conf., 4-5 December 1986, St. Petersburg Beach, FL, pp. $65-78$ January 1987

HOER, C. A.

Multiport Network Analyzers

McGraw-Hill Yearbook of Science and Technol., pp. 289-292 1986

CLAGUE, F. R.; LARSEN, N. T.

A Transient Response Error in Microwave Power Meters Using Thermistor Detectors

Dig., 28th Auto. RF Tech. Group Conf., 4-5 December 1986, St. Petersburg Beach, FL, pp. 79-89

December 1986

ENGEN, G. F.

On-Line Accuracy Assessment for the Dual Six-Port ANA: Background and Theory

Dig., 1986 Conf. on Prec. Electromagn. Meas., 23-27 June 1986, NBS/Gaithersburg, MD, p. 236

June 1986

ENGEN, G. F.

In Search of a More Realistic Accuracy Statement for Microwave Metrology

Dig., 27th Auto. RF Techniques Group, 5-6 June 1986, Baltimore, MD, pp. 181-183 June 1986

HOER, C. A.; ENGEN, G. F.

Calibrating a Dual Six-Port or Four-Port for Measuring Two-Ports with Any Connectors

Dig., 1986 IEEE Microwave Theory Tech. Symp., 2-4 June 1986, Baltimore, MD, pp. 665-668

June 1986 
HOER, C. A.

On-Line Accuracy Assessment for the Dual Six-Port ANA; Treatment of Systematic Errors

Dig., 1986 Conf. on Prec. Electromagn. Meas., 23-27 June 1986, NBS, Gaithersburg, MD, pp. 238-239

June 1986

HOER, C. A.; ENGEN, G. F.

On-Line Accuracy Assessment for the Dual Six-Port ANA: Extension to Non-Mating Connectors

Dig., 1986 Conf. on Prec. Electromagn. Meas., 23-27 June 1986, NBS, Gaithersburg, MD pp. 241-242

June 1986

JUDISH, R. M.

On-Line Accuracy Assessment for the Dual Six-Port ANA: Statistical Methods for Random Errors

Dig., 1986 Electromagn. Meas., 23-27 June 1986, NBS, Gaithersburg, MD, p. 237 June 1986

JUROSHEK, J. R.

On-Line Accuracy Assessment for the Dual Six-Port ANA: Experimental Results

Dig., 1986 Conf. on Prec. Electromagn. Meas., 23-27 June 1986, NBS, Gaithersburg, MD, p. 240

June 1986

ADAIR, R. T.; REEVE, G. R.; GATTERER, L. E.

Millimeter Wave Standards: An Emerging Need

Proc., IEEE Instrum. and Meas. Technol. Conf., 25-27 March 1986, Boulder, CO, Vol. IM-35, No. 4, pp. 376-382

March 1986

GINLEY, R. A.; ALLRED, C. M.

1.25 MHz Attenuation Measurement System

Proc., IEEE Instrum. and Meas. Technol. Conf., 25-27 March 1986, Boulder, CO, Vol. IM-35, No. 4, pp. 463-466

March 1986

MAJOR, J. R.; LIVINGSTON, E. M.; ADAIR, R. T.

Automatic Frequency Response of Frequency Modulated Generators Using the Bessel Null Method NBS TN 1093

March 1986

JUDISH, R. M.

Quality Control of Measurements--Measurement Assurance

Proc., IEEE Spec. Issue on Radio Meas. Methods and Standards, Vol. 74, No. 1, pp. 23-25 January 1986

SLADEK, M. J.; JESCH, R. L.

Standardization of Coaxial Connections in the IEC

Proc., IEEE Spec. Issue on Radio Meas. Methods and Standards, pp. 14-18 January 1986

HOLT, D. R.; HOER, C. A.

Estimation of True Power Ratios in Six-port Network Analyzers Using Diode Detectors

IEEE Trans. Instrum. Meas., Vol. IM-34, No. 4, pp. 558-563

December 1985 
WEIDMAN, M. P.

Finline Diode Six-Port: Fundamentals and Design Information

NBS TN 1090

December 1985

SEQUEIRA, H. B.; YATES, B. C.

Approach for Evaluating Effects of Wall Losses on Quarter-Wave Losses on Quarter-Wave Short-Circuit Impedance Standards

IEEE Trans. Microwave Theory Tech., Vol. MTT-33, No. 11, pp. 1106-1109 November 1985

JUROSHEK, J. R.; HOER, C. A.

A Technique for Extending the Dynamic Range of the Dual Six-Port Network Analyzer

IEEE Trans. Microwave Theory Tech., Vol. MTT-33, No. 6, pp. 453-459 June 1985

MAJOR, J. R.; LIVINGSTON, E. M.; ADAIR, R. T.

Automatic Frequency Response of Frequency Modulated Generators Using the Bessel Null Method Proc., 1985 Test and Measurement World Expo, 14-16 May 1985, San Jose, CA, pp. 78-100 May 1985

HOLT, D. R.; HOER, C. A.

Estimation of True Power Ratios in Six-port Network Analyzers Using Diode Detectors

Proc., 1985 Instrum. and Meas. Technol. Conf., 20-22 March 1985, Tampa, FL, pp. 140-141

March 1985

REEVE, G. R.; MILLER, C. K. S.

Current NBS Metrology Capabilities and Limitations at Millimeter Wave Frequencies

Proc., 1985 Meas. Science Conf., 17-18 January 1985, Santa Clara, CA, pp. 296-314

January 1985

MAJOR, J. R.; LIVINGSTON, E. M.; ADAIR, R. T.

Automatic Frequency Response of Frequency Modulated Generators Using the Bessel Null Method

Dig., 24th Auto. RF Tech. Group Conf., 5-6 December 1984, Columbia, MD, pp. 131-153

December 1984

JUDISH, R. M.; JONES, R. N.

A Generalized Method for the Calibration of Four-Terminal-Pair Type Digital Impedance Meters NBSIR 84-3016 August 1984

JUROSHEK, J. R.; HOER, C. A.

A High-Power Automatic Network Analyzer for Measuring the RF Power Absorbed by Biological Samples in a TEM Cell

IEEE Trans. Microwave Theory Tech., Vol. MTT-32, No. 8, pp. 818-824 August 1984

JUROSHEK, J. R.; HOER, C. A.

A Dual Six-Port Network Analyzer Using Diode Detectors

IEEE Trans. Microwave Theory Tech., Vol. MTT-32, No. 1, pp. 78-82

January 1984 
KAMPER, R. A.; HOER, C. A.

Millimeter Wave Standards at the National Bureau of Standards (NBS)

Proc., The Intl. Soc. for Optical Engineering (SPIE), 23-24 August 1983, San Diego, CA, Vol.

423, pp. 144-146

August 1983

ENGEN, G. F.

Redundancy: A Monitor of Six-Port Performance

Dig., IEEE 1983 Colloquium on Advances in S-Parameter Measurement at Microwave Lengths,

23 May 1983, Savoy Place, London, England, No. 1983/53, pp. 4-1 through 4-2

May 1983

HOER, C. A.

Choosing Line Lengths for Calibrating Network Analyzers

IEEE, Trans. Microwave Theory Tech., Vol. MTT-31, No. 2, pp. 76-78

January 1983

KAMPER, R. A.

Current Trends in NBS Calibration Services

NCSL Newsletter, Vol. 22, No. 1, pp. 38-39

March 1982

EBBESEN, H.; ENGEN, G. F.

Singularities in Calibration of Six-Port Network Analyzers

Dig., 1981 IEEE MTT-S Intl. Microwave Symp., p. 149

1981

HOER, C. A.

A High Power Dual Six-Port Automatic Network Analyzer Used in Determining Biological Effects of RF and Microwave Radiation

IEEE Trans. Microwave Theory Tech., Vol. MTT-29, pp. 157-159

December 1981

WEIDMAN, M. P.

WR-10 Single Six-Port Measurement System

NBSIR 81-1650

September 1981

WEIDMAN, M. P.; HUDSON, P. A.

WR-10 Millimeter Wave Microcalorimeter

NBS TN 1044

June 1981

ENGEN, G. F.

A Least Squares Solution for Use in Six-Port Measurement Technique

IEEE Trans. Microwave Theory Tech., Vol. MTT-28, No. 12, pp. 1473-1477

December 1980

JONES, R. N.

Evaluation of Three-Terminal and Four-Terminal Pair Capacitors at High Frequencies

NBS TN 1024

September 1980 
HILL, D. A.; WAIT, J. R.

Electromagnetic Theory of the Loosely Braided Coaxial Cable: Part II - Numerical Results IEEE Trans. Microwave Theory Tech., Vol. MTT-28, No. 4, pp. 326-331 April 1980

HILL, D. A.; WAIT, J. R.

Propagation Along a Coaxial Cable with a Helical Shield

IEEE Trans. Microwave Theory Tech., Vol. MTT-28, No. 2, pp. 84-89 February 1980

ENGEN, G. F.; HOER, C. A.

Through-Load-Delay: An Improved Technique for Calibrating the Dual Six-Port Automatic Network Analyzer

Dig., Intl. Microwave Symp., Orlando, FL, p. 53 1979

REEVE, G. R.; ARTHUR, M. G.

A Standard for RF Modulation Factors

NBS TN 1016

September 1979

HOER, C. A.

Performance of a Dual Six-Port Automatic Network Analyzer

Proc., IEEE MTT-S 1979, Intl. Microwave Symp.: The World of Microwaves, 3 April-2 May

1979, Orlando, FL, IEEE Cat. No. 79CHI439-9 MTT-S

April 1979

JONES, R. N.

A Comparison of Centrifuge and Freezing Calorimeter Methods for Measuring Free Water in Snow NBSIR 79-1604

April 1979

HOER, C. A.

Calibrating a Six-Port Reflectometer with Four Impedance Standards

NBS TN 1012

March 1979

ENGEN, G. F.; HOER, C. A.; SPECIALE, R. A.

The Application of "Through-Short-Delay" to the Calibration of the Dual Six-port

IEEE Intl. Microwave Symp. Dig., pp. 184-185

1978

KOMAREK, E. L.

Advantages of the Six-port Reflectometer for RF/Microwave Power Measurement in Operational

Systems

Government Micro-Circuit Applications Conf. Dig.

1978

DRIVER, L. D.; RIES, F. X.; REBULDELA, G.

NBS RF Voltage Comparator

NBSIR 78-871

December 1978 
ENGEN, G. F.

Calibrating the Six-Port Reflectometer by Means of Sliding Terminations

IEEE Trans. Microwave Theory Tech., Vol. MTT-26, No. 12, pp. 951-957

December 1978

KAMPER, R. A.

Status of RF and Microwave Calibration Services at NBS

Nat1. Conf. of Standards Laboratories Newsletter, Vol. 18, No. 3, p. 40

December 1978

HOER, C. A.

Calibrating Two Six-Port Reflectometers with Only One Impedance Standard

NBS TN 1004

June 1978

HOER, C. A.

Calibrating Two Six-Port Reflectometers With an Unknown Length of Precision Transmission Line

Proc., 1978 IEEE MTT-S Intl. Microwave Symp., 27-29 June 1978, Ottawa, Canada,

pp. 176-178

June 1978

ENGEN, G. F.

The Six-Port Measurement Technique: A Status Report

Microwave J., Vol. 21, pp. 18, 21-22, 24, 84, 87, 89

May 1978

ENGEN, G. F.

Advances in Microwave Measurement Science

Proc., IEEE, Vol. 66, pp. 374-384

April 1978

ENGEN, G. F.

Instrumentation: Six Ports Simplify Network

Microwave Syst. News, pp. 54-55

January 1978

ENGEN, G. F.

An Improved Circuit for Implementing the Six-Port Technique of Microwave Measurements

IEEE Trans. Microwave Theory Tech., Vol. MTT-25, No. 12, pp. 1080-1083 December 1977

HOER, C. A.

A Network Analyzer Incorporating Two 6-Port Reflectometers

IEEE Trans. Microwave Theory Tech., Vol. MIT-25, No. 12, pp. 1070-1074

December 1977

JESCH, R. L.

Evaluation of Low-Loss/Low-Reflection Two-Port Devices or Adapters by Automated Measurement Techniques

NBSIR 78-870

December 1977 
KOMAREK, E. L.

Performance Characteristics of an Automated Broad-Band Bolometer Unit Calibration System IEEE Trans. Microwave Theory, Vol. MTT-25, No. 12, pp. 1122-1127

December 1977

WEIDMAN, M. P.

A Semi-Automated Six-Port for Measuring Millimeter Wave Power and Complex Reflection Coefficient IEEE Trans. Microwave Theory Technol., Vol. MTT-25, No. 12, pp. 1083-1085 December 1977

LARSEN, N. T.

NBS Type IV RF Power Meter Operation and Maintenance

NBSIR 77-866

October 1977

ENGEN, G. F.

Design Considerations for Automatic Network Analyzers Based on the Six-Port Concept

Proc., IEE Euromeas 1977 Conf., 1-9 September 1977, Sussex, England; London, England, pp. $110-111$

September 1977

ENGEN, G. F.

An Improved Circuit for Implementing the Six-Port Technique of Microwave Measurements

1977 IEEE-MTT-S Intl. Microwave Symp., 21-23 June 1977, San Diego, CA, pp. 53-55 June 1977

ENGEN, G. F.

The Six-Port Reflectometer: An Alternative Network Analyzer

Proc., 1977 IEEE-MTT-S Intl. Microwave Symp., 21-23 June 1977, San Diego, CA, pp. 44-46 June 1977

HOER, C. A.

A Microwave Network Analyzer Using Two 6-Port Reflectometers;

Proc., 1977 IEEE-MTT-S Intl. Microwave Symp., 21-23 June 1977, San Diego, CA, pp. 47-49 June 1977

KAMPER, R. A.

The National Electromagnetic Measurement System

NBSIR 75-936

June 1977

KOMAREK, E. L.

An Application of the Six-Port Junction to Precision Measurement of Microwave One-Port Parameters

Proc., 1977 IEEE-MTT-S Intl. Microwave Symp., $21-23$ June 1977, San Diego, CA, pp. 56-57 June 1977

WEIDMAN, M. P.

A Semi-Automated Six-Port for Measuring Millimeter Wave Power and Complex Reflection Coefficient Proc., 1977 IEEE-MTT-S Intl. Microwave Symp., 21 -23 June 1977, San Diego, CA, pp. 58-60 June 1977 
ENGEN, G. F.

Determination of Microwave Phase and Amplitude from Power Measurements

IEEE Trans. Instrum. Meas., Vol. IM-25, pp. 414-418

December 1976

ESTIN, A. J.

Scattering Parameters of SMA Coaxial Connectors

IEEE Trans. Instrum. Meas., Vol. IM-25, No. 4, pp. 329-334

December 1976

JESCH, R. L.

Repeatability of SMA Coaxial Connectors

IEEE Trans. Instrum. Meas., Vol. IM-25, No. 4, pp. 314-320

December 1976

\section{LARSEN, N. T.}

A New Self-Balancing DC-Substitution RF Power Meter

IEEE Trans. Instrum. Meas., Vol. IM-25, No. 4, pp. 343-347

December 1976

RIES, F. X.

An International Intercomparison of Voltage Standards at $1 \mathrm{GHz}$ in Coaxial Transmission Line

IEEE Trans. Instrum. Meas., Vol. IM-25, No. 3, pp. 254-255

September 1976

ROE, K. C.; HOER, C. A.

A Microwave Vector Voltmeter System

NBSIR 76-844

August 1976

SIMPSON, P. A.; HUDSON, P. A.

Implementation of the Notch Technique as an RF Peak Pulse Power Standard

NBS TN 682

July 1976

ENGEN, G. F.

Measurement of Complex Microwave Circuit Parameters Using Only Power Detectors

Proc., 1976 Conf. on Prec. Electromagn. Meas., 28 June-1 July 1976, Boulder, CO, p. 171

June 1976

HOER, C. A.; ROE, K. C.; ALLRED, C. M.

Measuring and Minimizing Diode Detector Nonlinearity

Proc., 1976 Conf. on Prec. Electromagn. Meas., 28 June-1 July 1976, Boulder, CO, pp. 108-109 June 1976

KOMAREK, E.L.

An Automated Broadband System for Measurement of One-Port Microwave Parameters

Proc., 1976 Conf. on Prec. Electromagn. Meas., 28 June-1 July 1976, Boulder, CO, pp. 167-170 June 1976

LARSEN, N. T.

A New Self-Balancing DC-Substitution RF Power Meter

Proc., 1976 Conf. on Prec. Electromagn. Meas., 28 June-1 July 1976, Boulder, CO, pp. 203-205 June 1976 
LITTLE, W. E.; WAKEFIELD, J. P.; HEIM, L.; ALLRED, C. M.; ZAPF, T. L.

An NBS Developed Automatic Network Analyzer

Proc., 1976 Conf. on Prec. Electromagn. Meas., 28 June-1 July 1976, Boulder, CO, pp. 382-385 June 1976

LITTLE, W. E.; YATES, B. C.

Reflection Coefficient Standards for Automated Network Analyzers

Proc., 1976 Conf. on Prec. Electromagn. Meas., 28 June-1 July 1976, Boulder, CO, pp. 128-129 June 1976

ALLRED, C. M.; MANNEY, C. H.

The Calibration and Use of Directional Couplers Without Standards

IEEE Trans. Instrum. Meas., Vol. IM-25, No. 1, pp. 84-89

March 1976

HUDSON, P. A.

The National Measurement System for Medical Ultrasonics

NBSIR 75-937

February 1976

ADAIR, R. T.

A Precision $30 \mathrm{MHz}$ Waveguide-Below-Cutoff Attenuator with an Absolute Electronic Readout, NBS Model XII

NBSIR 76-833

January 1976

ENGEN, G. F.

Automated Calibration of Directional-Coupler-Bolometer-Mount Assemblies

IEEE Trans. Microwave Theory Tech., Vol. MTT-23, No. 12, pp. 984-990

December 1975

HOER, C. A.; ROE, K. C.

Using an Arbitrary Six-Port Junction to Measure Complex Voltage Ratios

IEEE Trans. Microwave Theory Tech., Vol. MTT-23, No. 12, pp. 978-984

December 1975

HOER, C. A.

Using Six-Port and Eight-Port Junctions to Measure Active and Passive Circuit Parameters NBS TN 673

September 1975

KANDA, M.; MAY, W. G.

A Millimeter Wave Reflection Beam Isolator

IEEE Trans. Microwave Theory Tech., Vol. MTT-23, No. 6, pp. 506-508

June 1975

ENGEN, G. F.

Automated Calibration of Directional-Coupler-Bolometer-Mount Assemblies

Proc., 1975 IEEE Microwave Theory and Technique Symp. Int., 12-14 May 1975, Palo Alto,

CA, pp. 98-99

May 1975 
JESCH, R. L.; HOER, C. A.

Characterization of a High Frequency Probe Assembly for Integrated Circuit Measurements NBS TN 663 January 1975

ENGEN, G. F.

Calibration Technique for Automated Network Analyzers with Application to Adapter Evaluation IEEE Trans. Microwave Theory Tech. Part II, 1974 Symp. Issue, Vol. MTT-22, pp. 1255-1260 December 1974

KOMAREK, E. L.; TRYON, P. V.

An Application of the Power Equation Concept and Automation Techniques to Precision Bolometer Unit Calibration

IEEE Trans. Microwave Theory Tech. Part II, 1974 Symp. Issue, Vol. MTT-22, pp. 1260-1267 December 1974

ENGEN, G. F.

An Alternative Calibration Technique for Automated Network Analyzers with Application to Adapter Evaluation

Proc., 1974 IEEE S-MTT Intl. Microwave Symp., 12-14 June 1974, Atlanta, GA, pp. 261-262 June 1974

HUDSON, P. A.; MELQUIST, D. G.; ONDREJKA, A. R.; WERNER, P. E.

Completion of the Program to Evaluate/Improve Instrumentation and Test Methods for Electroexplosive Device Safety Qualification

NBSIR 74-379

June 1974

JONES, R. N.

The Measurement of Lumped Parameter Impedance

NBS MN 141

June 1974

KOMAREK, E. L.

An Application of the Power Equation Concept and Automation Techniques to Precision Bolometer Unit Calibration

Proc., 1974 IEEE S-MTT Intl. Microwave Symp., 12-14 June 1974, Atlanta, GA, pp. 263-265 June 1974

SIMPSON, P. A.

Broadband Pulsed/CW Calibration Signal Standard for Field Intensity Meter (FIM) Receivers

NBSIR 74-371

June 1974

ENGEN, G. F.

Comments on Practical Analysis of Reflectometers and Power Equation Concepts

IEEE Trans. Instrum. Meas., Vol. IM-23, pp. 104-105

March 1974

LARSON, W.; CAMPBELL, E.

Microwave Attenuation Measurement System (Series Substitution)

NBS TN 647

February 1974 
ENGEN, G. F.

Calibration of an Arbitrary Six-Port Junction for Measurement of Active and Passive Circuit Parameters IEEE Trans. Instrum. Meas., Vol. IM-22, pp. 295-299 December 1973

WEIDMAN, M. P.; ENGEN, G. F.

Application of a Non-Ideal Sliding Short to Two-Port Loss Measurement NBS TN 644

October 1973

YATES, B. C.; COUNAS, G. J.

Summary of WR 15 Flange Evaluation at $60 \mathrm{GHz}$

NBS TN 642

October 1973

ENGEN, G. F.

Mismatch Considerations in Evaluating Amplifier Noise Performance

IEEE Trans. Instrum. Meas., Vol. IM-22, pp. 274-278

September 1973

ALLRED, C. M.; MANNEY, C. H.

Self-Calibration of Complex Ratio Measuring System

Proc., 1973 Intl. Conf. Meas. and Instrum., 17-23 June 1973, Dresden, Germany, Acta

Imeko Vol. 1, pp. 157-166

June 1973

HOER, C. A.; ENGEN, G. F.

Analysis of a Six-Port Junction for Measuring, V, I, A, B, Z, Gamma, and Phase

Proc., 1973 Intl. Conf. Meas. and Instrum., 17-23 June 1973, Dresden, Germany, Acta Imeko,

Vol. 1, pp. 213-222

June 1973

ENGEN, G. F.

Theory of UHF and Microwave Measurements Using the Power Equation Concept

NBS TN 637

April 1973

NAHMAN, N. S.

A Note on the Transition (Rise) Time Versus Line Length in Coaxial Cables

IEEE Trans. Circuit Theory, Vol. CT-20, pp. 165-167 March 1973

ENGEN, G. F.; HOER, C. A.

Application of an 'Arbitrary' 6-Port Junction to Power Measurement Problems

IEEE Trans. Instrum. Meas., Vol. IM-21, pp. 470-474

November 1972

HOER, C. A.

The Six-Port Coupler: A New Approach to Measuring Voltage, Current, Power, Impedance, and Phase IEEE Trans. Instrum. Meas., Vol. IM-21, pp. 466-470

November 1972 
FOOTE, W. J.; HUNTER, R. D.

Improved Gearing for Rotary-Vane Attenuators

Rev. Scientific Instrum., Vol. 43, No. 7, pp. 1042-1043

July 1972

YATES, B. C.; LARSON, W.

Millimeter Attenuation and Reflection Coefficient Measurement System

NBS TN 619

July 1972

ENGEN, G. F.; HOER, C. A.

Application of an 'Arbitrary' 6-Port Junction to Power Measurement Problems

Proc., 1972 Conf. on Prec. Electromagn. Meas., 26-29 June 1972, Boulder, CO, pp. 100-101 June 1972

ESTIN, A. J.

A Calculable Microwave Attenuation Standard: The High Q Cavity

Proc., 1972 Conf. on Prec. Electromagn. Meas., 26-29 June 1972, Boulder, CO, pp. 100-101 June 1972

HOER, C. A.

The Six-Port Coupler; A New Approach to Measuring V, I, P, Z, and Theta

Dig., 1972 Conf. on Prec. Electromagn. Meas., 26-29 June 1972, Boulder, CO, pp. 6-29 June 1972

HUDSON, P. A.; SAULSBERY, L. F.

An Adjustable-Slot-Length UHF Coaxial Coupler with Decade Bandwidth

IEEE Trans. Microwave Theory Tech., Vol. MTT-19, pp. 781-783

September 1971

ENGEN, G. F.; HUDSON, P. A.

International Intercomparison of Power Standards at $3 \mathrm{GHz}$

IEEE Trans. Microwave Theory Tech., Vol. MTT-19, pp. 411-413

April 1971

COOK, C. C.; ALLRED, C. M.

An Excitation System for Piston Attenuators

IEEE Trans. Instrum. Meas., Vol. IM-20, pp. 10-16

February 1971

ENGEN, G. F.

Theorem Giving Limits for $\mathrm{S}_{22}$ When $\mathrm{S}_{11}$ and the Two-Port Efficiency are Known

IEEE Trans. Instrum. Meas., Vol. IM-20, p. 78

February 1971

ENGEN, G. F.

Power Equations: A New Concept in the Description and Evaluation of Microwave Systems

IEEE Trans. Instrum. Meas., Vol. IM-20, pp. 49-57

February 1971

ENGEN, G. F.

A New Method of Characterizing Amplifier Noise Performance

IEEE Trans. Instrum. Meas., Vol. IM-19, pp. 344-349

November 1970 
HOER, C. A.; AGY, D. L.

Broad-Band Resistive-Divider-Type Directional Coupler

IEEE Trans. Instrum. Meas., Vol. IM-19, pp. 336-343

November 1970

CRAWFORD, M. L.; SMART, G. R.

New Coaxial Thermistor Mounts for Use as Precision Transfer Standards

Proc., 1970 Instrument Soc. of America Conf., 26-29 October 1970, Philadelphia, PA, N708-70, pp. $1-6$

October 1970

SIMPSON, P. A.; ONDREJKA, A. R.

Improvements to the NBS RF Peak-Pulse Power Standard

1970 Instrument Soc. of America Silver Jubilee Intl. Conf. and Exhibit, October 1970,

Philadelphia, PA, Paper 709-70, pp. 1-7

October 1970

WEIDMAN, M. P.; CAMPBELL, E.

A Method for Designing Multi-Screw Waveguide Tuners

NBS TN 393

October 1970

AGY, D. L.; NELSON, R. E.

An Immittance Transcomparator

1970 Annual Precision Measurement Association Metrology Conf. (3rd), 17-18 June 1970,

Gaithersburg, MD, Vol. 1, pp. 147-154

June 1970

SCOTT, W. W., Jr.

A New Coaxial RF-DC Ammeter

Proc., 1970 Conf. on Prec. Electromagn. Meas., 2-5 June 1970, Boulder, CO, Spec. Issue Conf.

on Prec. Electromagn. Meas., p. 13

June 1970

JONES, R. N.; HUNTLEY, L. E.

A Precision, High Frequency Calibration Facility for Coaxial Capacitance Standards

NBS TN 386

March 1970

LAWTON, R. A.; ALLRED, C. M.; HUDSON, P. A.

A Wide Range CW Power Measurement Technique

IEEE Trans. Instrum. Meas., Vol. IM-19, pp. 28-34

February 1970

ENGEN, G. F.

An Introduction to the Description and Evaluation of Microwave Systems Using Terminal Invariant

Parameters

NBS MN 112

October 1969

BEATTY, R. W.

Impedance Measurements and Standards for Uniconductor Waveguide

Proc., IEEE Spec. Issue on Radio Meas. Methods and Standards, Vol. 55, No. 6, pp. 933-941 June 1967 
ELLERBRUCH, D. A.

UHF and Microwave Phase-Shift Measurements

Proc., IEEE Spec. Issue on Radio Meas. Methods and Standards, Vol. 55, No. 6, pp. 960-969 June 1967

HUDSON, P. A.

Measurement of RF Peak Pulse Power

Proc., IEEE Spec. Issue on Radio Meas. Methods and Standards, Vol. 55, No. 6, pp. 851-855 June 1967

HUNTLEY, L. E.; JONES, R. N.

Lumped Parameter Impedance Measurements

Proc., IEEE Spec. Issue on Radio Meas. Methods and Standards, Vol. 55, No. 6, pp. 900-911 June 1967

JESCH, R. L.

Impedance Measurements in Coaxial Waveguide Systems

Proc., IEEE Spec. Issue on Radio Meas. Methods and Standards, Vol. 55, No. 6, pp. 912-923 June 1967

KERNS, D. M.

Definitions of v, i, Z, Y, a, b, $\Gamma$, and S

Proc., IEEE Spec. Issue on Radio Meas. Methods and Standards, Vol. 55, No. 6, pp. 892-900 June 1967

ONDREJKA, A. R.

Peak Pulse Voltage Measurement (Baseband Pulse)

Proc., IEEE Spec. Issue on Radio Meas. Methods and Standards, Vol. 55, No. 6, pp. 882-885 June 1967

RUMFELT, A. Y.; ELWELL, L. B.

Radio Frequency Power Measurements

Proc., IEEE Spec. Issue on Radio Meas. Methods and Standards, Vol. 55, No. 6, pp. 837-850 June 1967

\section{RUSSELL, D. H.; LARSON, W.}

RF Attenuation

Proc., IEEE Spec. Issue on Radio Meas. Methods and Standards, Vol. 55, No. 6, pp. 942-959 June 1967

SCOTT, W. W., Jr.; FREDERICK, N. V.

The Measurement of Current at Radio Frequencies

Proc., IEEE Spec. Issue on Radio Meas. Methods and Standards, Vol. 55, No. 6, pp. 886-891 June 1967

SELBY, M. C.

Voltage Measurement at High and Microwave Frequencies in Coaxial Systems

Proc., IEEE Spec. Issue on Radio Meas. Methods and Standards, Vol. 55, No. 6, pp. 877-882 June 1967 


\section{NOISE}

RANDA, J.

Uncertainties in NIST Noise-Temperature Measurements

NIST TN 1502

March 1998

WAIT, D. F.; RANDA, J.

Amplifier Noise Measurements at NIST

IEEE Trans. Instrum. Meas., Vol. 46, No. 2, pp. 482-485

April 1997

RANDA, J.

Noise Temperature Measurements on Wafer

NIST TN 1390

March 1997

WAIT, D. F.

Radiometer Equation for Noise Comparison Radiometers

IEEE Trans. Instrum. Meas., Vol. 44, No. 2, pp. 336-339

April 1995

PUCIC, S. P.

Uncertainties of the NIST Coaxial Noise Calibration System

Dig., 1994 Conf. on Prec. Electromagn. Meas., 27 June-1 July 1994, Boulder, CO, pp. 254-255 June 1994

WAIT, D. F.

Relative Accuracy of Isolated and Unisolated Noise Comparison Radiometers

Dig., 1994 Conf. on Prec. Electromagn. Meas., 27 June-1 July 1994, Boulder, CO, pp. 256-257 June 1994

PUCIC, S. P.

Evaluation of Uncertainties of the Null-Balanced Total-Power Radiometer System NCS1

NIST JRES, Vol. 99, No. 1, pp. 65-75

January-February 1994

PUCIC, S. P.

A Null-Balanced Total-Power Radiometer System NCS1

NIST JRES, Vol. 99, No. 1, pp. 45-53

January-February 1994

PUCIC, S. P.

Derivation of the System Equation for Null-Balanced Total-Power Radiometer System NCS1

NIST JRES, Vol. 99, No. 1, pp. 55-63

January-February 1994

PUCIC, S. P.

Diffusion of Copper into Gold Plating

Record, IEEE Instrum. Meas. Technol. Conf., 18-20 May 1993, Irvine, CA, pp. 114-117

May 1993

ENGEN, G. F.; WAIT, D. F.

A "Terminal Invariant" Description of Amplifier Noise

Dig., 1992 Conf. on Prec. Electromagn. Meas., 9-12 June 1992, Paris, France, pp. 250-251 June 1992 
PERERA, S.

"Broadband Mismatch Error" in Noise Measurement Systems

Dig., 1992 Conf. on Prec. Electromagn. Meas., 9-12 June 1992, Paris, France, pp. 256-257 June 1992

WAIT, D. F.

Measuring Accuracies for Various Techniques for Measuring Amplifier Noise

Dig., 39th Auto. RF Tech. Group Conf., 5 June 1992, Albuquerque, NM, pp. 43-52 June 1992

WAIT, D. F.

A Comparison of Three Techniques for the Precision Measurement of Amplifier Noise

Dig., 1992 Conf. on Prec. Electromagn. Meas., 9-12 June 1992, Paris, France, pp. 252-253 June 1992

DAYWITT, W. C.

Exact Principal Mode Field for a Lossy Coaxial Line

IEEE Trans. Microwave Theory Tech., Vol. MTT-39, No. 8, pp. 1313-1322 August 1991

WAIT, D. F.; ENGEN, G. F.

Application of Radiometry to the Accurate Measurement of Amplifier Noise

IEEE Trans. Instrum. Meas., Vol. IM-40, No. 2, pp. 433-437

April 1991

WAIT, D. F.; COUNAS, G. J.; KESSEL, W.; BUCHHOLZ, F. IM.

PTB - NIST Bilateral Comparison of Microwave Noise Power in Coaxial Transmission Line IEEE Trans. Instrum. Meas., Vol. IM-40, No. 2, pp. 449-454 April 1991

DAYWITT, W. C.

First Order Symmetric Modes for a Slightly Lossy Coaxial Transmission Line

IEEE Trans. Microwave Theory Tech., Vol. MTT-38, No. 11.pp. 1644-1650 November 1990

WAIT, D. F.

Comments Concerning On-Wafer Noise Parameter Measurements

Dig., 36th Auto. RF Tech. Group Conf., 29-30 November 1990, Monterey, CA, pp. 5-15 November 1990

DAYWITT, W. C.

First Order Principal Mode Fields and Distributed Line Parameters for a Slightly Lossy Coaxial Transmission Line.

Dig., Conf. on Prec. Electromagn. Meas., 11-14 June 1990, Ottawa, Canada, pp. 404-405 June 1990

PERERA, S.

A Simple Noise Calibration Radiometer

Dig., Conf. on Prec. Electromagn. Meas., 11-14 June 1990, Ottawa, Canada, pp. 32-33 June 1990 
WAIT, D. F.; COUNAS, G. J.; KESSEL, W.; BUCHHOLZ, F. IM.

PTB - NIST Bilateral Comparison of Microwave Noise Power in Coaxial Transmission Line

Dig., Conf. on Prec. Electromagn. Meas., 11-14 June 1990; Ottawa, Canada, pp. 34-35 June 1990

DAYWITT, W. C.; COUNAS, G. J.

Measuring Adapter Efficiency Using a Sliding Short Circuit

IEEE Trans. Microwave Theory Tech., Vol. MTT-38, No. 3, pp. 231-237

March 1990

DAYWITT, W. C.

Radiometer Equation and Analysis of systematic Errors for the NIST Automated Radiometers NIST TN 1327

March 1989

WAIT, D. F.; DAYWITT, W. C.

Intercomparison of NBS Noise Calibration Services

Dig., Conf. on Prec. Electromagn. Meas., 7-10 June 1988, Tsukuba Science City, Japan, pp. 209-210

1988

GEYER, R. G.

Magnetostatic Measurements for Mine Detection

NISTIR 88-3098

October 1988

DAYWITT, W. C.

Complex Admittance of a Lossy Coaxial Open Circuit with a Hollow Center Conductor

Intl. J. Sci. Metrol., Metrologia, Vol. 24, pp. 13-22

1987

DAYWITT, W. C.

A Simple Technique for Determining Joint Losses on a Coaxial Line from Swept-Frequency Reflection Data

IEEE Trans. Instrum. Meas., Vol. IM-36, pp. 468-473 June 1987

DAYWITT, W. C.

A Simple Technique for Investigating Defects in Coaxial Connectors

IEEE Trans. Microwave Theory Tech., Vol. MTT-35, No. 4, pp. 460-464 April 1987

WAIT, D. F.

The Impact of Automation on NBS Noise Temperature Measurements

Proc., IEEE Spec. Issue Radio Meas. Methods Standards, pp. 117-120 1986

DAYWITT, W. C.

A Simple Technique for Determining Joint Losses on a Coaxial Line from Swept-Frequency Reflection Data

Dig., 1986 Conf. on Prec. Electromagn. Meas., 23-27 June 1986, NBS, Gaithersburg, MD, p. 40 June 1986 
DAYWITT, W. C.

10-60 GHz G/T Measurements Using the Sun as a Source--A Preliminary Study

NBSIR 86-3046

April 1986

DAYWITT, W. C.

Broadband Noise Source Applications

Proc., 1985 Instrum. and Meas. Technol. Conf., 20-22 March, 1985, Tampa, FL, pp. 165-166 1985

DAYWITT, W. C.

A Derivation for the Noise Temperature of a Horn-type Noise Standard

Metrologia, 21 pp. 127-133

1985

HALFORD, D.

Transparent Metrology of Signal to Noise Ratios of Noisy Band-limited Digital Signals

NBS TN 1077

June 1985

PERERA, S.

Noise Temperature Measurements at the National Bureau of Standards

Proc., 1985 Instrum. and Meas. Technol. Conf., 20-22 March, 1985, Tampa, FL, pp. 159-160 March 1985

DAYWITT, W. C.

An Error Analysis for the Use of Presently Available Lunar Radio Flux Data in Broadbeam Antenna-System Measurements

Proc., 1984 Antenna Applications Symp., 19-21 September 1984, University of Illinois,

Urbana-Champaign, IL, pp. 1-26

September 1984

DAYWITT, W. C.

A Preliminary Investigation into Using the Sun as a Source for G/T Measurements

NBSIR 84-3015

August 1984

MILLER, C. K. S.; DAYWITT, W. C.

The Reference Noise Standards NBS WR62 and WR90

NBSIR 84-3005

May 1984

MILLER, C. K. S.; DAYWITT, W. C.

The NBS Switching Radiometers

NBSIR 84-3004

May 1984

DAYWITT, W. C.

A Coaxial Noise Standard for the $1 \mathrm{GHz}$ to $12.4 \mathrm{GHz}$ Frequency Range NBS TN 1074

March 1984 
DAYWITT, W. C.

An Error Analysis for the Use of Presently Available Lunar Radio Flux Data in Broadbeam Antenna-System Measurements

NBS TN 1073

February 1984

COUNAS, G. J.

NBS $2.0 \mathrm{GHz}$ to $4.0 \mathrm{GHz}$ Automated Radiometer Operation and Service Manual

NBSIR 84-1697

January 1984

DAYWITT, W. C.

Design and Error Analysis for the WR10 Thermal Noise Standard

NBS TN 1071

December 1983

WAIT, D. F.; DAYWITT, W. C.

Preliminary Examination of $20 \mathrm{GHz}$ G/T Measurements of Earth Terminals NBSIR 83-1686

March 1983

WAIT, D. F.

Precision Measurement of Antenna System Noise Using Radio Stars

IEEE Trans. Instrum. Meas., Vol. IM-32, No. 1, pp. 110-116

March 1983

WAIT, D. F.

Earth Terminal Measurement System Operations Manual (Revised)

NBSIR 83-1679 (Restricted)

January 1983

WAIT, D. F.

Precision Measurement of Antenna System Noise Using Radio Stars

Dig., 1982 Conf. on Prec. Electromagn. Meas., 28 June-1 July 1982, Boulder, CO, p. F-17 June 1982

COUNAS, G. J.; BREMER, T. H.

NBS 30/60 Megahertz Noise Measurement System Operation and Service Manual

NBSIR 81-1656

December 1981

WAKEFIELD, J.P.

Addendum to Earth Terminal Measurement System Maintenance Manual

NBSIR 81-1641

October 1981

ESTIN, A. J.; DAYWITT, W. C.

Evaluation of Signal Plus Noise Detection Error in an Envelope Detector with Logarithmic Compression IEEE Trans. Inf. Theory, Vol. IT-17, No. 5, pp. 663-664

September 1981 
ESTIN, A. J.; DAYWITT, W. C.

Evaluation of Signal Plus Noise Detection Error in an Envelope Detector with Logarithmic Compression NBSIR 79-1614 (Restricted)

August 1979

ESTIN, A. J.; DAYWITT, W. C.

Evaluation of Signal Plus Noise Detection Error in an Envelope Detector with Logarithmic Compression Proc., 1978 Conf. on Prec. Electromagn. Meas., 26-29 June 1978, Ottawa, Canada 1978

DAYWITT, W. C.

An Error Analysis for the Measurement of Satellite EIRP using a Calibrated Radio Star

IEEE Trans. Instrum. Meas., Vol. IM-27, No. 3, pp. 253-258

September 1978

DAYWITT, W. C.

Atmospheric Propagation Equations Used in the NBS Earth Terminal Measurement System NBSIR 78-883

April 1978

WAIT, D. F.

Earth Terminal Measurement System Operations Manual

NBSIR 78-879

April 1978

DAYWITT, W. C.

Error Equations Used in the NBS Earth-Terminal Measurement System

NBSIR 78-869

December 1977

DAYWITT, W. C.

A Precision Earth-Terminal System for Accurate C/KT, G/T, and EIRP Measurements with a Calibrated Radio Star

Proc., 1977 Union Radio Scientifique Internationale Intl. Symp. on Meas. in

Telecommunications, 3-7 October 1977, Lannion, France, pp. 1-4

October 1977

KANDA, M.

An Improved Solid State Noise Source

IEEE Trans. Microwave Theory Tech. pp. 173-182

December 1976

DAYWITT, W. C.

Error Equations Used in the NBS Precision G/T Measurement System

NBSIR 76-842

September 1976

KANDA, M.

Study of Errors in Absolute Flux Density Measurements of Cassiopeia A

IEEE Trans. Instrum. Meas., Vol. IM-25, No. 3, pp. 173-182

September 1976 
KANDA, M.

An Improved Solid State Noise Source

Proc., 1976 IEEE MTT-S Microwave Symp., 14-16 June, 1976, Cherry Hill, NJ, pp. 224-226 June 1976

KANDA, M.; LARSON, T. R.

Stability of Silicon Avalanche Noise Diodes at $\mathrm{K}$ and $\mathrm{Ka}$ Bands

Proc., 1976 Conf. on Precision Electromagn. Meas., 28 June-1 July 1976, Boulder, CO June 1976

KANDA, M.

Study of Errors in Absolute Flux Density Measurements of Cassiopeia A

NBSIR 75-822

October 1975

DAYWITT, W. C.; KANDA, M.

G/T Measurement Errors with Radio Stars

Proc., 1975 IEEE Intl. Antenna and Propagat. Symp., 2-4 June 1975, Urbana-Champaign, IL, Session 20, pp. 460-463

June 1975

KANDA, M.; LARSON, T. R.

A Measure for the Stability of Solid State Noise Sources

Proc., 1975 IEEE Intl. Microwave Theory and Technique Symp., 12-14 May 1975, Palo Alto, CA, pp. 315-317

May 1975

WAIT, D. F.; KANDA, M.; DAYWITT, W. C.; MILLER, C. K. S.

A Study of the Measurement of G/T Using Cassiopeia A

NBSIR 74-382

October 1974

ARTHUR, M. G.

The Measurement of Noise Performance Factors: A Metrology Guide

NBS MN 142

June 1974

DAYWITT, W. C.

A Reference Noise Standard for Millimeter Waves

IEEE Trans. Microwave Theory Tech., Vol. MTT-21, No. 12, pp. 845-847

December 1973

ENGEN, G. F.

Mismatch Considerations in Evaluating Amplifier Noise Performance

IEEE Trans. Instrum. Meas., Vol. IM-22, pp. 274-278

September 1973

HALFORD, D.

Comparing Frequencies

Physics Today, Vol. 26, No. 2, p. 15

February 1973 
BOYLE, D. R.; CLAGUE, F. R.; REEVE, G. R.; WAIT, D. F.; KANDA, M.

An Automated Precision Noise Figure Measurement System at $60 \mathrm{GHz}$

IEEE Trans. Instrum. Meas., Vol. IM-21, No. 4, pp. 543-549

November 1972

HALFORD, D.

Infrared-Microwave Frequency Synthesis Design: Some Relevant Conceptual Noise Aspects

Proc., 1971 Frequency Standards and Metrology Seminar, 30 August-1 September 1972, Quebec, Canada, pp. 431-466

August 1972

DAYWITT, W. C.; FOOTE, W. J.; CAMPBELL, E.

WR15 Thermal Noise Standard

NBS TN 615

March 1972

ARTHUR, M. G.

A Precision HF-Noise Power Measurement System

Proc., Instrum. Soc. Amer. ISA-70 Conf., October 1971 October 1971

\section{ENGEN, G. F.}

A New Method of Characterizing Amplifier Noise Performance

IEEE Trans. Instrum. Meas., Vol. IM-19, pp. 344-349

November 1970 


\section{REMOTE SENSING}

GANS, W. L.; GEYER, R. G.; KLEMPERER, W. K.

Quantifying Standard Performance of Electromagnetic-Based Mine Detectors

NISTIR 3982

October 1991

HILL, D. A.

Magnetic Dipole Excitation of an Insulated Conductor of Finite Length

IEEE Trans Geosci. Remote Sensing, Vol. GE-28, No. 3, pp. 289-294

May 1990

HILL, D. A.; ANDERSON, L. R.

Propagation Along a Two-Wire Line Located at the Air-Earth Interface

IEEE Trans. Geosci. Remote Sensing, Vol. GE-28, No. 3, pp. 400-402

May 1990

HILL, D. A.

Magnetic Dipole Excitation of a Long Conductor in a Lossy Medium

IEEE Trans. Geosci. Remote Sensing, Vol. GE-26, No. 6, pp. 720-725

November 1988

HILL, D. A.

Fields of Horizontal Currents Located Above the Earth

IEEE Trans. Geosci. Remote Sensing, Vol. GE-26, No. 6, pp. 726-732

November 1988

HIILL, D. A.

Electromagnetic Wave Propagation in a Symmetrical Coal Seam

IEEE Trans. Antennas Propagat., Vol. AP-34, No. 2, pp. 244-247

February 1986

HILL, D. A.

Radio Propagation in a Coal Seam and the Inverse Problem

Proc., 1985 Intl. Symp. on Antennas and EM Theory, 26-28 August 1985, Beijing, China, pp. 422-427

August 1985

BELSHER, D. R.; MCLAUGHLIN, R. G.; REPJAR, A. G.; BUSSEY, H. E.

Detection of Lost Wells and Unknown Water-Filled Voids in Coal Mines through Development of an

Experimental Microwave System

NBSIR 84-3017

September 1984

HILL, D. A.

Radio Propagation in a Coal Seam and the Inverse Problem

NBS JRES, Vol. 89, No. 5, pp. 385-394

September/October 1984

CHEW, $\mathrm{H}$.

Electromagnetic Modeling of Oil Shale Retorts for Remote Sensing Purposes

IEEE Trans. Geosci. Remote Sensing, Vol. GE-20, No. 4, pp. 510-517

October 1982 
ROE, K. C.; WITTMANN, R. C.

Improved Coal Interface Detector

NBSIR 82-1663

May 1982

CHEW, H.

Modeling of Oil Shale Retorts for Electromagnetic Sensing Techniques

NBSIR 81-1653

November 1981

CHANG, A. T. C.; SHIUE, J. C.; BOYNE, H. S.; ELLERBRUCH, D. A.; COUNAS, G. J.

Preliminary Results of Passive Microwave Snow Experiment During February and March 1978

NASA-TP-1408, Rept-7802-F22, p. 114

1979

BELSHER, D. R.

Feasibility Study on the Use of a Microwave System for the Nondestructive Evaluation of Historic Adobe Structures

NBSIR 79-1610

July 1979

MONTGOMERY, J. P.; CHANG, D. C.

Electromagnetic Boundary-Value Problems Based Upon a Modification of Residue Calculus and

Function Theoretic Techniques

NBS MN 164

June 1979

BOYNE, H. S.; ELLERBRUCH, D. A.

Microwave Measurements of Snow Stratigraphy and Water Equivalence

Proc., Western Snow Conf.

April 1979

ELLERBRUCH, D. A.; JOHNSON, R. B.; BELSHER, D. R.; YAGHJIAN, A. D.; STEPPE, M. C.;

FLEMING R. W.

High Resolution Sensing Techniques for Slope Stability Studies

Federal Highway Administration Report, FHWA-7-3-0001

March 1979

ELLERBRUCH, D. A.; JESCH, R. L.; JONES, R. N.; BUSSEY, H. E.; BOYNE, H. S.

Electromagnetic Scattering Properties of Soils and Snow

Proc., 1978 Intl. Symp. on Remote Sensing of Environment (12th), 20-26 April 1978,

Ann Arbor, MI, Vol. 2, pp. 957-974

April 1978

ELLERBRUCH, D. A.; BELSHER, D. R.

Electromagnetic Technique of Measuring Coal Layer Thickness

IEEE Trans. Geosci. Electron., Vol. GE-16, No. 2, pp. 126-133

April 1978

BEREUTER, W. A.; CHANG, D. C.

Electromagnetic Remote Sensing of Inhomogeneous Media

NBSIR 76-851

January 1977 
ELLERBRUCH, D. A.; BELSHER, D. R.

FM-CW Electromagnetic Technique of Measuring Coal Layer Thickness

NBSIR 76-840

May 1976

ELLERBRUCH, D. A.; ADAMS, J. W.

Microwave Measurement of Coal Layer Thickness

NBSIR 74-387

September 1974

ELLERBRUCH, D. A.

Electromagnetic Attenuation Properties of Clay and Gravel Soils NBSIR 74-381

August 1974

STOLTENBERG, R. E.

Detection of Human Intruders by Low Frequency Sonic Interferometric Techniques NBSIR 74-364

May 1974

CRUZ, J. E.; ROGERS, E. H.; HIESTER, A. E.

Continuous Liquid Level Measurements with Time-Domain Reflectometry Adv. Cryo. Eng., Vol. 18, pp. 323-327 (Paper H-4)

1973

COLLIER, R. S.; ELLERBRUCH, D. A.

RF (Radiofrequency) Total Mass Gauging in Large Storage Containers: Empty Tank Modes NBSIR 73-346

October 1973

COLLIER, R. S.; ELLERBRUCH, D. A.; CRUZ, J. E.; STOKES, R. W.; LUFT, P. E. Mass Quantity Gauging by RF Mode Analysis

NBSIR 73-318

June 1973 
JOHNK, R. T.; ONDREJKA, A. R.

The Extraction of Electrical Matereial Properties Using a Free-Space Time-Domain RF Absorber Reflectivity Measurement System

Proc., IEEE EMC Symp., 18-22 August 1997, Austin TX, pp. 537-542

August 1997

JARGON, J. A.; JANEZIC, M. D.

Measuring Complex Permittivity and Permeability Using Time Domain Network Analysis Proc., 1996 IEEE MTT-S Intl. Microwave Symp., 17-21 June 1996, San Francisco, CA, pp. $1407-1410$

June 1996

DEGROOT, D. C.; MARKS, R. B.

Optimizing Time-Domain Network Analysis

Dig., 46th Auto. RF Tech. Group Conf., 30 Nov.-1 Dec. 1995, Scottsdale, AZ, pp. 19-28 December 1995

MARKS, R. B.; DEGROOT, D. C.; JARGON, J. A.

High-Speed Interconnection Characterization Using Time Domain Network Analysis Advancing Microelectronics, Vol. 22, No. 6, pp. 35-39

November/December 1995

MARKS, R. B.; HAYDEN, L. A.; RETTIG, J. B.

Accuracy and Repeatability in Time Domain Network Analysis

Proc., 44th Auto. RF Tech. Group Conf., 1-2 December 1994, Boulder, CO, pp. 39-46

December 1994

MARKS, R. B.; HAYDEN, L. A.; JARGON, J. A.; WILLIAMS, D. F.

Time Domain Network Analysis Using the Multiline TRL Calibration

Proc., 44th Auto. RF Tech. Group Conf., 1-2 December 1994, Boulder, CO, pp. 47-55

December 1994

HAYDEN, L. A.; MARKS, R. B.

Accuracy in Time Domain Transmission Line Measurements

Proc., 3rd Topical Mtg. on Elect. Perf. of Electronic Packaging, November 2-4, 1994, Monterey, CA, pp. 176-178

November 1994

BLEJER, D. J.; WITTMANN, R. C.; YAGHJIAN, A. D.

On-Axis Fields from a Circular Uniform Surface Current

Proc., Ultra-Wideband Short-Pulse Electromagnetics Intl Conf., 8-10 October 1992, Brooklyn, NY, pp. 285-292

1993

JOHNK, R. T.; ONDREJKA, A. R.; TOFANI, S.; KANDA, M.

Time-Domain Measurements of the Electromagnetic Backscatter of Pyramidal Absorbers and Metallic

Plates

IEEE Trans. Electromagn. Compat., Vol. 35, No. 4, pp. 429-433

November 1993

ALLEN, O. E.; HILL, D. A.; ONDREJKA, A. R.

Time-Domain Antenna Characterizations

IEEE Trans. Electromagn. Compat., Vol. 35, No. 3, pp. 339-346

August 1993 
KANDA, M.; ONDREJKA, A. R.

An Ultra-Broadband and Nondispersive Sensor for the Measurement of Time-Domain Signals

Proc., Symp. on Electromagnetic Security for Information Protection, 21-22 November 1991,

Rome, Italy, pp. 65-78

November 1991

TOFANI, S.; ONDREJKA, A. R.; KANDA, M.

A Time-Domain Method for Characterizing the Reflection Coefficient of Absorbing Materials from 30 to $1000 \mathrm{MHz}$

IEEE Trans. Electromagn. Compat., Vol. 33, No. 3, pp. 234-240 August 1991

GANS, W. L.; GEYER, R. G.; KLEMPERER, W. K.

Suggested Methods and Standards for Testing and Verification of Electromagnetic Buried Object Detectors

NISTIR 88-3915

March 1990

ADAMS, J. W.; ONDREJKA, A. R.; CAVCEY, K. H.; CRUZ, J. E.; MEDLEY, H. W.;

GROSVENOR, J. H.

Recent Improvements in Time-Domain EMC Measurement System

NISTIR 89-3927

November 1989

GEYER, R. G.

Magnetostatic Measurements for Mine Detection

NISTIR 88-3098

October 1988

ADAMS, J. W.; ONDREJKA, A. R.; MEDLEY, H. W.

Time-Domain System for Indentification of the Natural Resonant Frequencies of Aircraft Relevant to Electromagnetic Compatibility Testing

NBSIR 87-3077

November 1987

LAWTON, R. A.; RIAD, S. M.; ANDREWS, J. R.

Pulse and Time Domain Measurements

Proc., IEEE, Spec. Issue on Radio Meas. Methods and Stds, Vol. 74, No. 1, pp. 77-81

January 1986

LAWTON, R. A.; ONDREJKA, A. R.

Standards for the Measurement of Impulsive Fields Radiated by a TEM Horn Antenna

Proc., 1977 Union Radio Scientifique Intl. E Comm., 3-7 October 1977, Lannion, France, pp. 1-4

1977

ANDREWS, J. R.

Impulse Generator Spectrum Amplitude Measurement Techniques

IEEE Trans. Instrum. Meas., Vol. IM-25, No. 4, pp. 380-384

December 1976 
ANDREWS, J. R.; GANS, W. L.

Time Domain Automatic Network Analyzer

L'Onde Electrique, Vol. 55, No. 10, pp. 569-574

December 1975

ANDREWS, J. R.; BALDWIN, E. E.

Baseband Impulse Generator Useful to $5 \mathrm{GHz}$

Proc., 1975 IEEE Intl. Symp. on Electromagn. Compat., 7-9 October 1975, San Antonio, TX, pp. 1-4

October 1975

GANS, W. L.; ANDREWS, J. R.

Time Domain Automatic Network Analyzer for Measurement of RF and Microwave Components NBS TN 672

September 1975

NAHMAN, N. S.; JICKLING, R. M.

Frequency Domain Measurement of Baseband Instrumentation NBSIR 73-330

July 1973

NAHMAN, N. S.; JICKLING, R. M.; HOLT, D. R.

Reference-Waveform Generation Using Debye Dielectric Dispersion

NBSIR 73-304

December 1972 


\section{WAVEFORM METROLOGY}

CHESNUT, S. M.; PAULTER, N. G., Jr.

Automatic Waveform Analysis and Measurement System User Manual

NISTIR 3978

December 1991

TOFANI, S.; ONDREJKA, A. R.; KANDA, M.

A Time-Domain Method for Characterizing the Reflection Coefficient of Absorbing Materials from 30 to $1000 \mathrm{MHz}$

IEEE Trans. Electromagn. Compat., Vol. 33, No. 3, pp. 234-240

August 1991

ONDREJKA, A. R.; KANDA, M.

A Time-Domain Method for Measuring the Reflection Coefficient of Microwave Absorbers at

Frequencies Below $1 \mathrm{GHz}$

Dig., 1991 Antennas and Propagation Society Symp., 24-28 June 1991, London, Ontario,

Canada, Vol. 3, pp. 1656-1663

June 1991

PAULTER, N. G., Jr.; MAJUMDAR, A.

A New Triple Correlation Technique for Measuring Ultrashort Laser Pulses

Rev. Sci. Instrum., Vol. 62, No. 3, pp. 567-578

March 1991

PAULTER, N. G., Jr.; MAJUMDAR, A.

A New Proposed Triple Correlator for the Measurement of Ultrashort Laser Pulses

Opt. Comm., Vol. 81, No. 1,2, pp. 95-100

February 1991

GANS, W. L.

Dynamic Calibration of Oscilloscopes and Waveform Recorders Using Pulse Standards

Record, Instrumentation and Measurement Technology (IMTC) Conf., 13-15 February 1990,

San Jose, CA, pp. 246-250

February 1990

LAWTON, R. A.; MEXER, K.

Waveform Standards for Electro-Optics: A Pulse Duration Comparison

IEEE Trans. Microwave Theory Tech., Vol. MTT-35, No. 4, pp. 450-453 April 1987

NAHMAN, N. S.

Software Correction of Measured Pulse Data

NATO ASI Series on Fast Electrical and Optical Meas., Vol. 1, Series E, No. 108, pp. 351-417 1986

GANS, W. L.

Picosecond Pulse Waveform Error Analysis at NBS

Proc., IEEE Spec. Issue on Radio Meas. Methods and Standards, pp. 86-90

January 1986

RIAD, S. M.

The Deconvolution Problem: An Overview

Proc., IEEE, Spec. Issue on Radio Meas. Methods and Standards, pp. 82-85

January 1986 
LAWTON, R. A.

An Efficient Antialiasing Filter

IEEE Trans. Instrum. Meas., Vol. IM-34, No. 4, pp. 570-573

December 1985

GANS, W. L.

Picosecond Pulse Measurements at NBS

Proc., 1985 Instrum. and Meas. Technol. Conf., 20-22 March 1985, Tampa, FL, pp. 142-144 March 1985

LAWTON, R. A.

Pulse Waveform Standards for Electro-Optics

Proc., 1985 Topical Meeting on Picosecond Electronics and Opto-Electronics, 13-15 March 1985, Lake Tahoe, NV, pp. 205-206

March 1985

LAWTON, R. A.

Status Report Transient Waveform Recorder Standard

Proc., 1985 Instrum. and Meas. Technol. Conf., 20-22 March 1985, Tampa, FL, pp. 154-155

March 1985

LAWTON, R. A.; NAHMAN, N. S.; BIGELOW, J. M.

A Solid State Reference Waveform Standard

IEEE Trans. Instrum. Meas., Vol. IM-33, No. 3, pp. 201-205

September 1984

LAWTON, R. A.

An Efficient Antialiasing Filter

Dig., 1984 Conf. on Prec. Electromagn. Meas., 20-24 August 1984, Delft, The Netherlands, pp. 104-105

August 1984

LAWTON, R. A.; NAHMAN, N. S.; BIGELOW, J. M.

A Solid State Reference Waveform Standard

Dig., Instrum. and Meas. Technol. Conf., 17-18 January 1984, Long Beach, CA, pp. 74-76 January 1984

ANDREWS, J. R.; BELL, B. A.; BALDWIN, E. E.

Reference Flat Pulse Generator

NBS TN 1067

October 1983

LAWTON, R. A.

Photoconductive Switches Used for Waveform Generation at the National Bureau of Standards

Proc., 1983 Picosecond Optoelectronics Conf., SPIE - The International Society for Optical

Engineering, 24-26 August 1983, San Diego, CA, Vol. 439, pp. 88-94

August 1983

ANDREWS, J. R.; BELL, B. A.; NAHMAN, N. S.; BALDWIN, E. E.

Reference Waveform Flat Pulse Generator

IEEE Trans. Instrum. Meas., Vol. IM-32, No. 1, pp. 27-32

March 1983 
GANS, W. L.

The Measurement and Deconvolution of Time Jitter in Equivalent-Time Waveform Samplers IEEE Trans. Instrum. Meas., Vol. IM-32, No. 1, pp. 126-133 March 1983

NAHMAN, N. S.

Picosecond Domain Waveform Measurements; Status and Future Directions

IEEE Trans. Instrum. Meas., Vol. IM-32, No. 1, pp. 117-124 March 1983

\section{LAWTON, R. A.}

Performance Standards for Waveform Recorders

IEEE Trans. Nucl. Sci., Vol. NS-30, No. 1, pp. 263-266

February 1983

ANDREWS, J. R.; NAHMAN, N. S.; BELL, B. A.

Status of Reference Waveform Standards Development at NBS

Proc., 1981 Waveform Recorder Seminar, 15 October 1981, Boulder, CO, NBS SP 634, pp. $69-88$

June 1982

ANDREWS, J. R.; BELL, B. A.; NAHMAN, N. S.; BALDWIN, E. E.

Reference Waveform Flat Pulse Generator

Dig., Conf. on Prec. Electromagn. Meas. June 1982

June 1982

\section{LAWTON, R. A.}

Precision Picosecond-Microsecond Electromagnetic Waveform Measurement at NBS

NBS SP 628, pp. 392-407

June 1982

\section{LAWTON, R. A.}

Measurement Needs and Techniques for Evaluation/Calibration,

Proc., 1981 Waveform Recorder Seminar, 15 October 1981, Boulder, CO, NBS SP 634 June 1982

NAHMAN, N. S.

Some Generic Waveform Recorder Problems

Proc., 1981 Waveform Recorder Seminar, 15 October 1981, Boulder, CO, NBS SP 634, pp. 1-5 June 1982

KANDA, M.

Time Domain Sensors and Radiators

Natl. Radio Sci. Meeting, January 1982, University of Colorado, Boulder, CO January 1982

GANS, W. L.; NAHMAN, N. S.

Shielded Balanced and Coaxial Transmission Lines - Parametric Measurements and Instrumentation

Relevant to Signal Waveform Transmission in Digital Service

NBSIR 81-1042

June 1981 
ANDREWS, J. R.; BALDWIN, E. E.

Amplitude Calibrator for Oscilloscopes

NBSIR 81-1646

April 1981

NAHMAN, N. S.

Error Criteria and the Use of Reference Waveforms

Electron. Test, Vol. 4, No. 2, pp. 72-76

February 1981

ANDREWS, J. R.

Pulse Reference Waveform Standards Development at NBS

Proc., ATE Seminar/Exhib., January 1981, Pasadena, CA, p. IV.13 through IV.19 January 1981

ANDREWS, J. R.

The Estimation of the Pulse Waveform in the Calibration of Impulse Generators

Dig., Intl. Union of Radio Sci. (Union Radio Scientifique Internationale) Meeting, January 1981, Boulder, CO, p. 87

January 1981

NAHMAN, N. S.; GUILLAUME, M. E.

Deconvolution of Duration Limited Waveforms

Dig., Union Radio Scientifique Internationale, January 1981, Boulder, CO, p. 86 January 1981

NAHMAN, N. S.

Error Criteria and the Use of Reference Waveforms

Proc. ATE Seminar/Exhib., January 1981, Pasadena, CA, pp. IV.21 through IV.28 January 1981

GANS, W. L.

Present Capabilities of the NBS Automatic Pulse Measurement System

Dig., Conf. on Prec. Electromagn. Meas., pp. 25-26

1976

NAHMAN, N.S.

Picosecond Domain Waveform Measurements (Japanese translation)

Nikkei Electron., Nikkei-McGraw Hill

April 1979

YOUNG, M.; LAWTON, R. A.

Measurement of Pulsed-Laser Power

NBS TN 1010

February 1979

NAHMAN, N.S.

Picosecond-Domain Waveform Measurements

Proc., IEEE, Vol. 66, pp. 414-423

April 1978 
ANDREWS, J. R.; NAHMAN, N. S.

The Measurement of Pulse Transition Duration

Proc., Union Radio Scientifique Internationale Symp., 3-7 October, 1977, Lannion, France, pp. 159-164

October 1977

\section{LAWTON, R. A.; YOUNG, M.}

Laser-Mode Beating Used for Detector Frequency-Response Measurements

Appl. Opt., Vol. 16, pp. 2703-2705

October 1977

\section{ANDREWS, J. R.}

Impulse Generator Spectrum Amplitude Measurement Techniques

Dig., Conf. on Prec. Electromagn. Meas., pp. 23-24

1976

RIAD, S. M.; NAHMAN, N. S.

Application of the Homomorphic Deconvolution for the Separation of TDR Signals Occurring in Overlapping Time Windows

Dig., Conf. on Prec. Electromagn. Meas. pp. 27-29

1976

\section{GANS, W. L.}

Present Capabilities of the NBS Automatic Pulse Measurement System

IEEE Trans. Instrum. Meas., IM-25, pp. 384-388

December 1976

RIAD, S. M.; NAHMAN, N. S.

Application of the Homomorphic Deconvolution for the Separation of TDR Signals Occurring in Overlapping Time Windows

IEEE Trans. Instrum. Meas., IM-25, pp. 388-391

December 1976

LAWTON, R. A.; ANDREWS, J. R.

Electrical Strobing of Photoconductor Cuts Sampling Oscilloscope's Risetime

Laser Focus, pp. 62-65

November 1976

ANDREWS, J. R.; LAWTON, R. A.

Electrically Strobed Optical Waveform Sampling Oscilloscope

Rev. Sci. Instrum., Vol. 47, pp. 311-313

March 1976

\section{ANDREWS, J. R.}

Measurement on Pulses and Pulse Transmission Media, Circuits and Components

Intl. Union of Radio Sci. (Union Radio Scientifique Internationale) Review of Radio Sci., p. 9 1975-1977

\section{ANDREWS, J. R.}

Directional-Coupler Technique for Triggering a Tunnel Diode

IEEE Trans. Instrum. Meas., IM-24, pp. 275-277

September 1975 
LAWTON, R. A.; ANDREWS, J. R.

Pulsed-Laser Application to Sampling Oscilloscope

Electron. Lett., Vol. 11, pp. 138

April 1975

ANDREWS, J. R.; GANS, W. L.

Pulsed Wavemeter Timing Reference for Sampling Oscilloscope Calibration

IEEE Trans. Instrum. Meas., IM-24, p. 82

March 1975

LAWTON, R. A.; SCAVENNEC, A.

Photoconductive Detector of Fast-Transition Optical Waveforms

Electron. Lett., Vol. 11, pp. 74-75

February 1975

ANDREWS, J. R.

Precision Picosecond-Pulse Measurements Using a High Quality Superconducting Delay Line IEEE Trans. Instrum. Meas., IM-23, pp. 468-472

December 1974

ANDREWS, J. R.; LAWTON, R. A.

Picosecond Pulse Research at NBS

Proc., Joint Meas. Conf., 12-14 November 1974, Gaithersburg, MD, pp. 123-140

November 1974

ANDREWS, J. R.

Precision Picosecond-Pulse Measurements Using a High Quality Superconducting Delay Line

Proc.Conf. on Prec. Electromagn. Meas., 1-5 July 1974, London, England, pp. 316-318 July 1974

SCAVENNEC, A.; NAHMAN, N.S.

Active and Passive Mode Locking of Continuously Operating Rhodamine 6G Dye Lasers

NBSIR 73-347

February 1974

ANDREWS, J. R.

Inexpensive Laser Diode Pulse Generator for Optical Waveguide Studies

Rev. Sci. Instrum., Vol. 45, pp. 22-24

January 1974

HONDA, T.; NAHMAN, N. S.

Application of Ultrashort Optical Pulses to Electrical Pulse Measurements

Proc. Symp. on New Instrum., 28-29 January 1974, IEE of Japan, Hokkaido University, pp. 29-38

January 1974

ANDREWS, J. R.

Random Sampling Oscilloscope for the Observation of Mercury Switch Closure Transition Times IEEE Trans. Instrum. Meas., IM-22, pp. 375-381

December 1973 
GANS, W. L.; NAHMAN, N. S.

Pulse Testing of RF and Microwave Components NBSIR 1973

July 1973

ANDREWS, J. R.

Random Sampling Oscilloscope Time Base

NBSIR 73-309

June 1973

HOLT, D. R.; NAHMAN, N. S.

Coaxial Line Pulse-Response Error Due to a Planar Skin Effect Approximation

IEEE Trans. Instrum. Meas., IM-21, pp. 515-519 November 1972

HONDA, T.; NAHMAN, N. S.

A Dye Modelocked Nd+3 Glass Laser for Generating Electrical Reference Waveforms NBSIR 74-360

September 1972

NAHMAN, N. S.; HOLT, D. R.

Transient Analysis of Coaxial Cables Using the Skin Effect Approximation A + B (the square root of s) IEEE Trans. Circuit Theory, CT-19, pp. 443-451

September 1972

BOUILLE, R.; ANDREWS, J. R.

Measurement of Broadening of Pulses in Glass Fibers

Electron. Lett., Vol. 8, pp. 309-310

June 1972

HOLT, D. R.; NAHMAN, N. S.

Coaxial Line Pulse-Response Error Due to a Planar Skin Effect Approximation

Proc., 1972 Conf. on Prec. Electromagn. Meas., 26-29 June 1972, Boulder, CO, pp. 1-2 June 1972

LAWTON, R. A.; NAHMAN, N. S.

Pyroelectric Detector Application to Baseband Pulse Measurements

Electron. Lett., Vol. 8, pp. 318-330

June 1972

ANDREWS, J. R.

A Frequency Calibrator for UHF Using an Avalanche Transistor

Q S T, Vol. 56, pp. 16-18

May 1972

ANDREWS, J. R.; WHITTEMORE, T.; MCCAA, W. D. Jr.

Survey of Present Waveform Sampling System Limitations

NBS Rept. 10731

February 1972

ANDREWS, J. R.

An Interfacing Unit for a $28 \mathrm{psec}$ Feedthrough Sampling Oscilloscope

Rev. Sci. Instrum., Vol. 42, pp. 1882-1885

December 1971 
ANDREWS, J. R.

Horizontal Time Base Sweep Generator for a Traveling Wave Oscilloscope

IEEE Trans. Nucl. Sci., NS-18, pp. 3-18

October 1971

EKSTROM, M. P.; MCCAA, W. D. Jr.; NAHMAN, N. S.

The Measured Time and Frequency Response of a Miniature Superconducting Coaxial Line

IEEE Trans. Nucl. Sci., NS-18, pp. 18-25

October 1971

ANDREWS, J. R.; NAHMAN, N.S.

Electron-Beam Deflection in Traveling-Wave Oscilloscopes

Record of Symp. on Electron, Ion, and Laser Beam Technol. (11th), 12-14 May 1971, Boulder, $\mathrm{CO}$

May 1971

MCCAA, W. D. Jr.; NAHMAN, N. S.

Generation of Reference Waveforms by Uniform Lossy Transmission Lines

IEEE Trans. Instrum. Meas., Vol. IM-19, pp. 382-390

November 1970

ANDREWS, J. R.

Improved Bias Supply for Tunnel-Diode Picosecond Pulse Generators

IEEE Trans. Instrum. Meas., Vol. IM-19, pp. 171-175

August 1970

NAHMAN, N. S.; EKSTROM, M. P.; MCCAA, W. D. Jr.

Measured Time and Frequency Response of a Miniature Superconducting Coaxial Line

Applied Superconductivity Conf., June 1970, Boulder, CO

June 1970 


\section{MISCELLANEOUS}

LYONS, R. M.

A Bibliography of the NIST Electromagnetic Fields Division Publications

NISTIR 5063

August 1997

STORK, F.J.B.; BEALL, J. A.; ROSHKO, A.; DEGROOT, D. C.; RUDMAN, D. A.; ONO, R. H.

Surface Resistance and Morphology of YBCO Films as a Function of Thickness

IEEE Trans. Appl. Superconduct., Vol. 7, No. 2, pp. 1921-1924 June 1997

LYONS, R. M.

A Bibliography of the NIST Electromagnetic Fields Division Publications

NISTIR 5050

August 1996

KNOPP, K. J.; CHRISTENSEN, D. H.; HILL, J. R.; MASTERSON, K. D.

Thin-Film Design for Enhanced Stability of Optically Pumped Vertical-Cavity Surface-Emitting Lasers (VCSELs)

Surface and Coatings Tech., Vol. 86-87, pp. 783-787 and Proc., Intl. Conf. Metallurgical

Coatings and Thin Films, 22-26 April 1966, San Diego, CA

April 1996

LYONS, R. M.

A Bibliography of the NIST Electromagnetic Fields Division Publications

NISTIR 5039

August 1995

REEVE, G. R.; FRIDAY, D. S.

NIST and the Navy-Past, Present and Future

Proc., Test and Calib. Symp., American Soc. Naval Engineers, 30 November-1 December 1994,

Washington, DC, pp. 229-237

December 1994

WITTMANN, R. C.; ALPERT, B. K.

An Integral Occurring in Coherence Theory

SIAM Review, Vol 36, No. 4, p. 655

December 1994

LYONS, R. M.; GIBSON, K. A.

A Bibliography of the NIST Electromagnetic Fields Division Publications

NISTIR 5028

September 1994

WEIL, C. M.

Electromagnetic Properties of Materials: The NIST Metrology Program

Proc., 5th DOD Electromagnetic Windows Symp., 19-21 October 1993, Boulder, CO, pp. 175-182

October 1993

YOUNG, M.; WITTMANN, R. C.

Vector Theory of Diffraction by a Single-Mode Fiber: Application to Mode-Field Diameter Measurements

Opt. Lett., Vol. 18, No. 20, pp. 1715-1717

October 1993 
LYONS, R. M.; GIBSON, K. A.

A Bibliography of the NIST Electromagnetic Fields Division Publications

NISTIR 5009

September 1993

LYONS, R. M.; GIBSON, K. A.

A Bibliography of the NIST Electromagnetic Fields Division Publications NISTIR 3993

August 1992

LYONS, R. M.; GIBSON, K. A.

A Bibliography of the NIST Electromagnetic Fields Division Publications NISTIR 3973

August 1991

LYONS, R. M.; GIBSON, K. A.

A Bibliography of the NIST Electromagnetic Fields Division Publications NISTIR 3945

August 1990

REEVE, G. R.; MARKS, R. B.; BLACKBURN, D.

MMIC Related Metrology at the National Institute of Standards and Technology

Record, Instrum. Meas. Tech. (IMTC) Conf.; 13-15 February 1990; San Jose, CA; pp. 196-199 February 1990

LYONS, R. M.; GIBSON, K. A.

A Bibliography of the NIST Electromagnetic Fields Division Publications NISTIR 89-3920

September 1989

FITZGERRELL, R. G.

Mobile Antennas

National Institute of Justice Std.-0205.01

May 1989

VANZURA, E. J.

Creating CSUBs in Basic, Part II

HP Design and Automation Magazine, p. 25

November 1988

VANZURA, E. J.

Creating CSUBs in BASIC, Part I

HP Design and Automation Magazine, pp. 18-21

October 1988

REIDY, A. R.; GIBSON, K. A.

A Bibliography of NIST Electromagnetic Fields Division Publications

NISTIR 88-3900

September 1988 


\section{Miscellaneous (cont.)}

VANZURA, E. J.

Creating CSUBs Written in FORTRAN That Run in BASIC

Proc., INTEREX Technical Computer Users Conf., 7-12 August 1988, Orlando, FL, paper 20, pp. 1-18

August 1988

JESCH, R. L.

Fixed and Base Station FM Receivers

National Institute of Justice Std. - 0206.01

July 1988

JESCH, R. L.

Fixed and Base Station FM Transmitters

National Institute of Justice Std. - 0201.01

September 1987

BENSEMA, W. D.

Mobile FM Transceivers

National Institute of Justice Std. - 0210.00

May 1986

JESCH, R. L.

Personal/Mobile FM Transceivers

National Institute of Justice Std. - 0224.00

May 1986

GIBSON, K. A.; PAGE, J. M.; MILLER, C. K. S.

A Bibliography of the NBS Electromagnetic Fields Division Publications

NBSIR 85-3040

February 1986

KU, H. H.; JUDISH, R. M.

Fundamentals of Error Analysis

Special Issue on Radio Measurements and Standards, Proc., Vol. 74, No. 1, pp. 25-27

January 1986

BENSEMA, W. D.

Personal FM Transceivers

National Institute of Justice Std. - 0209.01

September 1985

JESCH, R. L.

Measured Vehicular Antenna Performance

IEEE Trans. Veh. Technol., Vol. VT-34, No. 2, pp. 97-107

May 1985

GIBSON, K. A.; MULLER, C. K. S.

A Bibliography of the NBS Electromagnetic Fields Division Publications

NBSIR 85-3022

April 1985 
LARSEN, N. T.; VECCHIA, D. F.; SUGAR, G. R.

VOR Calibration Services

NBS TN 1069

April 1985

KUFFEL, J.; MALEWSKI, R.; VAN HEESWIJK, R.; LAWTON, R. A.

Dynamic Performance of Digital Recorders Used for Monitoring High Voltage Impulse Tests

Proc., 1985 Instrum. and Meas. Technol. Conf., 20-22 March 1985, Tampa, FL, pp. 211-215 March 1985

HILL, D. A.

Book Review - Leaky Feeders and Subsurface Radio Communications

IEEE Antennas Propagat., Vol. IM-25, No. 3, pp. 23-24

June 1983

JESCH, R. L.

Dielectric Measurements of Oil Shale as Functions of Temperature and Frequency

NBSIR 83-1683

January 1983

HILL, D. A.

HF Ground Wave Propagation over Forested and Built-up Terrain

NTIA Report 82-114

December 1982

CHEW, $\mathbf{H}$.

Electromagnetic Modeling of Oil Shale Retorts for Remote Sensing Purposes

IEEE Trans. Geosci. Remote Sensing, Vol. GE-20, No. 4, pp. 510-517

October 1982

GIBSON, K. A.; MILLER, C. K. S.

Bibliography of the Electromagnetic Fields Division Publications

NBSIR 82-1673

August 1982

STOLTENBERG, R. E.; WITTMANN, R. C.

Acoustical Interferometer Wader AIW Final Report of Advanced Development Test and Evaluation

NBSIR 82-167 (Restricted)

June 1982

ROE, K. C.; WITTMANN, R. C.

Improved Coal Interface Detector

NBSIR 82-1663

May 1982

HILL, D. A.; WAIT, J. R.

Ground Wave Propagation over a Mixed Path with an Elevation Change

IEEE Trans. Antennas Propagat., Vol. AP-30, No. 1, pp. 139-141

January 1982

NELSON, R. E.; JCKLING, R. M.

Control Cable Assemblies Used with Mobile FM Receivers

National Institute of Justice Std. - 0216.00

December 1981 
CHEW, H.

Modeling of Oil Shale Retorts for Electromagnetic Sensing Techniques NBSIR 81-1653

November 1981

YAGHJIAN, A. D.

Augmented Electric- and Magnetic-Field Integral Equations

Radio Sci., Vol. 16, No. 6, pp. 987-1001

November-December 1981

HILL, D. A.; WAIT, J. R.

HF Radio Wave Transmission over Sea-Ice and Remote Sensing Possibilities

IEEE Trans. Geosci. Remote Sensing, Vol. GE-19, No. 4, pp. 204-209

October 1981

HILL, D. A.; WAIT, J. R.

HF Ground Wave Propagation over Mixed Land, Sea, and Sea-Ice Paths

IEEE Trans. Geosci. Remote Sensing, Vol. GE-19, No. 4, pp. 210-216

October 1981

WAKEFIELD, J. P.

Addendum to Earth Terminal Measurement System Maintenance Manual

NBSIR 81-1641

October 1981

CLARK, H. E.

Requirements for an Effective National Nonionizing Radiation Measurement System NBS SP 613

June 1981

LEWIS, R. L.

Spherical Scanning Data Processing: An Algorithm for Halving the Data Processing Effort When the Radiation into the Back Hemisphere is Negligible

Dig., 1981 IEEE Antenna Propagat. Soc. Intl. Symp., June 1981

June 1981

YAGHJAN, A. D.

A Delta-Distribution Derivation of the Electric Field in the Source Region

Dig., 1981 IEEE Antenna Propagat. Soc. Intl. Symp., June 1981

June 1981

HILL, D. A.; WAIT, J. R.

HF Ground Wave Propagation over Sea Ice for a Spherical Earth Model

IEEE Trans. Antennas Propagat., Vol. AP-19, No. 3, pp. 525-527

May 1981

YAGHJAN, A. D.

Reply to Criticism on Electric Dyadic Functions in the Source Region

Proc., IEEE, Vol. 69, No. 2

February 1981 
BOWMAN, R. R.

A Temperature Probe for Radio-Frequency Heated Material

NBSIR 81-1634

January 1981

ELLERBRUCH, D. A.; BOYNE, H. S.

Active Microwave Water Equivalence Measurements

Proc., Ft. Collins Snow Workshop

1980

HILL, D. A.; WAIT, J. R.

Electromagnetic Characteristics of a Coaxial Cable with Periodic Slots

IEEE Trans. Electromagn. Compat., Vol. EMC-22, No. 4, pp. 303-307

November 1980

MILLER, C. K. S.

A Bibliography of Publications in the NBS Electromagnetic Fields Division

NBSIR 80-1635

November 1980

JONES, R. N.

Evaluation of Three-Terminal and Four-Terminal Pair Capacitors at High Frequencies NBS TN 1024

September 1980

WAIT, J. R.; HILL, D. A.

Fields of a Horizontal Loop of Arbitrary Shape Buried in a Two-Layer Earth

Radio Sci., Vol. 15, No. 5, pp. 903-912

September-October 1980

YAGHJIAN, A. D.

Augmented Electric- and Magnetic-Field Integral Equations

Dig., Union Radio Scientifique Internationale Symp. on EM Waves, Munich Germany August 1980

DRIVER, L. D.; RIES, F. X.

A Wideband RF Voltage Comparator

Dig., 1980 Conf. Precision Electromagn. Meas., 23-27 June 1980, Braunschweig, FDR, pp. 487-488

June 1980

HILL, D. A.; WAIT, J. R.

Ground Wave Attenuation Function for a Spherical Earth with Arbitrary Surface Impedance Radio Sci.

Radio Sci., Vol. 15, No. 3, pp. 637-643

May-June 1980

HILL, D. A.; WAIT, J. R.

On the Excitation of the Zenneck Surface Wave Over the Ground at $10 \mathrm{MHz}$

Annales des Telecommunications, Vol. 35, Nos. 5-6, pp. 1-4

May-June 1980 
HILL, D. A.; WAIT, J. R.

Electromagnetic Theory of the Loosely Braided Coaxial Cable: Part II - Numerical Results IEEE Trans. Microwave Theory Technol., Vol. MTT-28, No. 4, pp. 326-331 April 1980

HILL, D. A.; WAIT, J. R.

Propagation Along a Coaxial Cable with a Helical Shield

IEEE Trans. Microwave Theory Technol., Vol. MTT-28, No. 2, pp. 84-89 February 1980

WYSS, J. C.; STWALLEY, W. C.; KOCH, M. E.; VERMA, K. K.

Laser Fluorescence Diagnostics of Lithium Vapor for Inertial Confinement Applications Topical Meeting on Inertial Confinement Fusion, February 1980, San Diego, CA February 1980

YAGHJIAN, A. D.

Electric Dyadic Green's Functions in the Source Region

Proc., IEEE, Vol. 68, No. 2

February 1980

HAMULTON, C.; LlOYD, F.; PETERSON, R.; ANDREWS, J. R.

A Superconducting Sampler for Josephson Logic Circuits

Appl. Phys. Lett., Vol. 35, No. 9, pp. 718-719

November 1979

WHARTON, M. J.; FRUSH, C. L.; NAHMAN, N. S.

A Video Recorder for Coherent Doppler Radar

IEEE Trans. Geosci. Electron., Special Issue on Radio Metrology, GE-17, No. 4, pp. 171-178 October 1979

ARTHUR, M. G.; REEVE, G. R.

Modulation Factor Standards

NBS TN 1016

September 1979

NAHMAN, N. S.; ALLRED, C. M.; ANDREWS, J. R.; HOER, C. A.; LAWTON, R. A.

Radar Absorber Measurement Techniques at Frequencies Above $20 \mathrm{GHz}$

NBSIR 79-1613

August 1979

MONTGOMERY, J. P.; CHANG, D. C.

Electromagnetic Boundary-Value Problems Based Upon a Modification of Residue Calculus and

Function Theoretic Techniques

NBS MN 164

June 1979

JONES, R. N.

A Comparison of Centrifuge and Freezing Calorimeter Methods for Measuring Free Water in Snow

NBSIR 79-1604

April 1979 
KING, J.; PEISER, H. S.; SANGSTER, R. C.

Guidelines for Specification and Procurement of Measurement Instrumentation NBSIR 78-891

June 1978

SANGSTER, R. C.

Collected Executive Summaries: Studies of the National Measurement System NBSIR 75-947-Rev. S.

February 1978

YAGHJIAN, A. D.

A Direct Approach to the Derivation of Electric Dyadic Green's Functions NBS TN 1000

January 1978

JESCH, R. L.

Evaluation of Low-Loss/Low-Reflection Two-Port Devices or Adapters by Automated Measurement Techniques

NBSIR 78-870

December 1977

JICKLING, R. M.; SHAFER, J. F.

FM Repeater Systems

National Institute of Justice Std. - 0213.00

November 1977

YOUNG, M.; LAWTON, R. A.

Saturation of Optical Detectors at High Modulation Frequency

Proc., Annual Meeting Opt. Soc. of America, 12 October 1977, Toronto, Canada, Vol. 67, p. 1398

October 1977

ZAPF, T. L.; HARVEY, M. E.; LARSEN, N. T.; STOLTENBERG, R. E.

Ultrasonic Calorimeter for Beam Power Measurements from 1 to 15 Megahertz

Proc., 1976 Ultrasonics Symp., 29 September-1 October 1977, Annapolis, MD, IEEE Cat 76,

CH1120-5SU, pp. 573-576

September 1977

NELSON, R. E.

A Guide to Voice Scramblers for Law Enforcement Agencies

NBS SP 480-8

December 1976

SANGSTER, R. C.

Study of the National Measurement System. Final Summary Report, 1972-75

NBSIR 75-925

December 1976

YAGHJAN, A. D.

A Direct Approach to the Derivation of Electric Dyadic Green's Functions

Dig., AP Symp., October 1976, pp. 76-156

October 1976 
SANGSTER, R. C.

Collected Executive Summaries: Studies of the National Measurement System 1972-75

NBSIR 75-947

September 1976

ZAPF, T. L.; HARVEY, M. E.; LARSEN, N. T.; STOLTENBERG, R. E.

Ultrasonic Calorimeter for Beam Power Measurements from 1 to 15 Megahertz NBS TN 686

September 1976

SANGSTER, R. C.

Transactions Matrix Description of the National System of Physical Measurements

NBSIR 75-943

August 1976

LAWTON, R. A.; ANDREWS, J. R.

Optically Strobed Sampling Oscilloscope

IEEE Trans. Instrum. Meas., Vol. IM-25, pp. 56-60

March 1976

KANDA, M.; MAY, W. G.

Reflection Beam Isolator for Submillimeter Wavelength

NASA-CR-140761

June 1975

KERNS, D. M.

Scattering-Matrix Description and Near-Field Measurements of Electroacoustic Transducers

J. Acoust. Soc. Amer., Vol. 57, No. 2, pp. 497-507

February 1975

JESCH, R. L.; HOER, C. A.

Characterization of a High Frequency Probe Assembly for Integrated Circuit Measurements NBS TN 663

January 1975

ROGERS, G. J.; SAWYER, D. E.; JESCH, R. L.

Semiconductor Measurement Technology: Measurement of Transistor Scattering Parameters NBS SP 400-5

January 1975

KANDA, M.; MAY, W. G.

Hollow-Cylinder Waveguide Isolators for use at Millimeter Wavelengths

NASA-CR-140760

November 1974

JICKLING, R. M.; SHAFER, J. F.

Repeaters for Law Enforcement Communication Systems-Law Enforcement Standard Program National Institute of Justice Std.-0206.00

October 1974

LITTLE, W. E.

Automated Computer Controlled Measurements

Proc., 1974 Conf. Preci. Electromagn. Meas., 1-5 July 1974, London, England

July 1974 
BUSSEY, H. E.; LARSEN, E. B.

Buried Antenna Performance: Development of Small Resonant Buried Antennas RADC-TR-74-169M, AD 783274

June 1974

JESCH, R. L.; BAILEY, R. A.; TAUSCH, H. J.

Characterization of A H.F. Probe for Integrated Circuits Measurements

Proc., Government Micro-Circuit Applications Conf., 25-27 June 1974, Boulder, CO, pp. 120-121

June 1974

WEITZEL, D. H.; LOWE, L. T.; ELLERBRUCH, D. A.; CRUZ, J. E.; SINDT, C. F.

Hydrogen Slush Density Reference System

NASA-CR-124764

June 1974

YAGHJIAN, A. D.

Direct Integration of the Field Equations for Electroacoustic Transducers

IEEE Lett., Vol. 62, No. 6, pp. 858-859

June 1974

STOLTENBERG, R. E.

Detection of Human Intruders by Low Frequency Sonic Interferometric Techniques

NBSIR 74-364

May 1974

MILLER, C. K. S.

Millimeter Wave Metrology Capabilities at NBS

Proc., 1974 Millimeter Waves Techniques Conf., 26-28 March 1974, San Diego, CA, p. 11 March 1974

YAGHJIAN, A. D.

Theory of Adjoint Reciprocity for Electroacoustic Transducers

NBSIR 73-329

February 1974

SCAVENNEC, A.; NAHMAN, N. S.

A Simple Passively Mode-Locked CW Dye Laser

IEEE J. Quantum Electron., Vol. QE-10, pp. 95-96

January 1974

BERRY, L. A.; FITZGERRELL, R. G.; VOGLER, L. E.

Investigation of Effect of Antenna Site and Antenna Type on LF Non-Directional Beacon Performance FAA-RD-73-174

1973

WAIT, D. F.; BEATTY, R. W.

The 1973 Intl. Microwave Symp.

IEEE Trans. Microwave Theory Technol., Vol. MTT-21, No. 12, pp. 747-751

December 1973 
ELLERBRUCH, D. A.

Test Results for the Mooring Line Data Line

NBSIR 73-341

October 1973

HALFORD, D.; SHOAF, J. H.; RISLEY, A. S.

Spectral Density Analysis: Frequency Domain Specification and Measurement of Signal Stability 1973 Annual Symp. on Frequency Control (27th), 12-14 June 1973, Cherry Hill, NJ, pp. 421-431

June 1973

SCOTT, W. W., Jr.

Batteries Used with Law Enforcement Communications Equipment: Chargers and Charging Techniques NBS Rept. 10732

June 1973

STOLTENBERG, R. E.

RF Null Detector NBS/SND

NBSIR 73-302

June 1973

ELLERBRUCH, D. A.

Application of Measurements of Electromagnetic Phenomena to Oceanography

Proc., 1972 UJNR/MEC Symp. (1st), Record of the U.S.-Japan UJNR Joint Symp. on Marine

Electronics, (Japanese Electrical Industrial Committee, Tokyo, Japan), 9 October 1972, Tokyo, Japan, pp. 23-30

October 1972

\section{HALFORD, D.}

Infrared-Microwave Frequency Synthesis Design: Some Relevant Conceptual Noise Aspects

Proc., 1971 Frequency Standards and Metrology Seminar, 30 August-1 September 1971,

Quebec, Canada, pp. 431-466

August 1972

SANGSTER, R. C.

Relevancy of Measurements by a Systems Approach

Proc., 1972 Joint Meas. Conf., 21-23 June 1972, Boulder, CO, pp. 31-37

June 1972

MANNEY, C. H.

Applicability of Speech Bandwidth Compression Techniques in Mine Electromagnetic Communications

NBS Rept. 10728

February 1972

ENGEN, G. F.

An Extension to the Sliding Short Method of Connector and Adaptor Evaluation

NBS JRES, Vol. 75C, pp. 177-183

1971

JESCH, R. L.; BERRY, I. S.

Batteries Used with Law Enforcement Communications Equipment

NBS Rept. 10722

October 1971 
KANDA, M.

Non-Reciprocal Devices Using Solid State Magnetoplasmas at Millimeter and Submillimeter

Wavelengths NASA-CR-122923

August 1971

COLLIER, R. S.; CRUZ, J. E.; ELLERBRUCH, D. A.; LOWE, L. T.; WEITZEL, D. H.

Instrumentation for Hydrogen Slush Storage Containers

NASA-CR-119241

June 1971

WEITZEL, D. H.; LOWE, L. T.; ELLERBRUCH, D. A.; CRUZ, J. E.; SINDT, C. F.

Hydrogen Slush Density Reference System

NBS Rept. 9796

June 1971

BRANDENBERGER, H.; HADORN, F.; HALFORD, D.; SHOAF, J. H.

High Quality Quartz Crystal Oscillators: Frequency Domain and Time Domain Stability

Proc., 1971 Frequency Control Annual Symp. (25th), 26-28 April 1971, Atlantic City, NJ, pp. 226-234

April 1971

TAGGART, H. E.; WORKMAN, J. L.; NELSON, R. E.

Emergency Locator Transmitters: Effective Radiated Power Levels and Techniques of Determining

Effective Radiated Power

NBS Rept. 9769

February 1971

\section{ELLERBRUCH, D. A.}

Microwave Methods for Cryogenic Liquid and Slush Instrumentation

IEEE Trans. Instrum. Meas., Vol. IM-19, No. 4, pp. 412-416

November 1970

SCOTT, w. W., Jr.

New Coaxial RF-DC Ammeter

IEEE Trans. Instrum. Meas., Vol. IM-19, No. 4, pp. 318-323

November 1970

LEWIS, R. L.

The Wave-Hop Fields for an Inclined Dipole Over a Spherical Earth with an Anisotropic Ionosphere

OT-ITR-RR5

October 1970

WEIDMAN, M. P.; CAMPBELL, E.

A Method for Designing Multi-Screw Waveguide Tuners

NBS TN 393

October 1970

FOOTE, W. J.

A New Flange Design for O-Ring Seals

Rev. Sci. Instrum.

August 1970 
Miscellaneous (cont.)

HAYES, R. E.; KANDA, M.; MASCLET, G.; MAY, W. G.

The Application of Semiconductors to Quasi-Optical Isolators for use at Submillimeter Wavelengths NASA-CR-86379

April 1970

WACKER, P. F.; CORD, M. S.; BURKHARD, D. G.; PETERSEN, J. D.; KUKOL, R. F.

Microwave Spectral Tables, Volume III, Polyatomic Molecules with Internal Rotation NBS MN 70, Vol 3.

June 1969 


\section{AUTHOR INDEX}

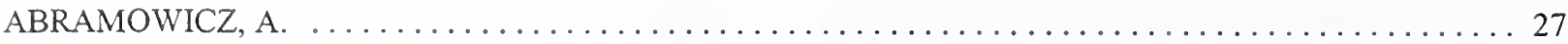

ADAIR, R. T.

ADAMS, J. W.

$83-87,93$

AGEE, D. A.

$40-42,44,46,47,49,51,53,54,57,58,61,67-73,109,111$

AGY, D. L. .

ALLEN, J. W.

ALLEN, O. E.

ALLEY, G. D.

ALLRED, C. M.

ALPERT, B. K.

ALSPACH, W. J.

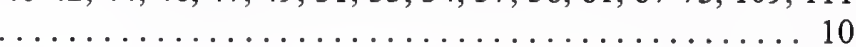

ANDERSH, D.

ANDERSON, L. R.

ANDERSON, W. T.

ANDREWS, J. R.

. 74

ANGLESIO, L.

ANSON, W. J.

ARTHUR, M. G.

$86,92,93,95-97,127$

$3-5,121$

AULD, B. A.

. 58

BABЛ, $\mathrm{T}$.

BACHMAN, D. D

BAILEY, R. A

BAIRD, R. C.

BAKER-JARVIS, J. R

BALDWIN, E. E.

BEALL, J. A.

$24,66-70,111,112,114-120,127,129$

BEAN, J. L.

$16,46,57$

BEATTY, R. W.

BEHE, R. .

BELL, B. A.

BELQUIN, J.

BELSHER, D. R.

$61,62,65,67,71,89,105,106,127$
$\ldots \ldots \ldots \ldots \ldots \ldots \ldots \ldots \ldots$

BENNETT, W. S.

BENSEMA, W. D.

18,60

BEREUTER, W. A.

BERRY, I. S.

$11,13,14,17-19,22,26,36,72$

BERRY, L. A

$66-68,112,114-116$

$66-68,112,114-116$

BIGELOW, J. M.

75,121

BLACK, D. N.

97,130

BLACKBURN, D.

BLEJER, D. J.

114,115

BLONDY, P.

BLOOMFIELD, R. A.

$65,70,107-109$

BORSERO, M.

BOUILLE, $\mathrm{R}$

BOWMAN, R. R.

BOYLE, D. R.

BOYNE, H.S.

BRANDENBERGER

....... 34

BREMER, T. H.

$17,19,45,46,49,51,56,59,62,68,70-72,123$

BUCHHOLZ, F. IM.

108

131

130

114

4,5

BUDLONG, A.

82,122

BURKHARD, D. G.

BURNS, J. G. 
BUSSEY, H. E. $26,33-35,107,108,130$

BUTLER, C. M. 36 CAMELL, D. G. $36-39,43,45,46$ CAMPBELL, E. $94,97,106,132$

CANALES, $\mathrm{S}$. $4,5,7-9$

CAPOBIANCO, T. E. . 31

CAVCEY, K. H.

CEREMUGA, J. $12,38,39,41,42,46,48,76,111$

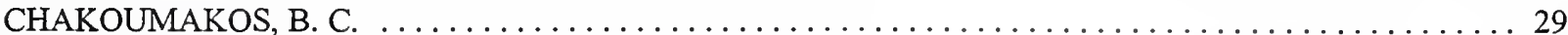

CHANG, A. T. C.

CHANG, D. C.

$17,18,45,46,48,49,55,59,61-67,69,108,127$

CHESNUT, S. M.

$107,108,124,125$

CHEW, $\mathrm{H}$.

$\ldots \ldots \ldots 121$

CHRISTENSEN, D. H.

$77-82,84,85,106$

CLARK, A. V.

CLARK, H. E.

CLEARY, J.

109,132

COOK, C. C.

CORD, M. S.

CORMACK, R. H.

COUNAS, G. J.

CRAWFORD, M. L.

CROS, D.

CRUZ, J. E.

D'AMORE, G.

DAMBRINE, G.

DAVIDSON, A.

DAY, G. W.

DAYWITT, W. C.

DECKER, W. F.

DeGroot, D. C

DELYSER, R. R.

DERZAKOWSKI, K.

81

DEVREEDE, J. P. M.

DOBROSKI, H.

$\ldots \ldots \ldots \ldots \ldots 22$

$13,16,17,22-25,33,77,100-106$

DOMICH, P. D.

$74,75,110,121$

DOUGHERTY, H. T.

DRIVER, L. D.

DUNGAN, D. F.

......... 36

EBBESEN, $\mathrm{H}$.

27,28

EHRET, R. L.

EKSTROM, M. P.

ELLERBRUCH, D. A.

ELWELL, L. B.

ENGEN, G. F.

ESTIN, A. J.

FEDOR, L. S.

FENTON, R.

FITZGERRELL, R. G

FLANIGAN, W. F. Jr.

$\ldots 4$

70

30-32

FLEMING R. W.

FOOTE, W. J.

. 22

$41,42,44-47,50,51,53,54,58,60,61,89,126$

FRANCIS, M. H. 
FREYER, G. J.

FRUSH, C. L.

FURLOW, R.

GALLIANO, P. G.

GANS, W. L

107, 111-119

GARY, J. M.

GATTERER, L. E.

GENTLE, D. G.

GERMAN, R. F.

GEYER, R. G.

$27-33,101,107,11$

GIBSON, K. A.

121-124

GILLILAND, D.

38,40

GINLEY, R. A.

$75,76,78,86$

GJERTSON, W.

38,40

GOLDFARB, R. B.

... 29

GREENE, F. M.

$68,70,71$

GREENLEE, D. H.

17,18

GRIMM, K. R.

$5-7,15$

GROSVENOR, J. H.

$28-32,111$

GUERRIERI, J. R

GUILLAUME, M. E

GUILLON, P.

GUPTA, K. C.

HADORN, F.

HALFORD, D.

$102,105,106,131,132$

HAMILTON, C. .

HARPER, J.

HARVEY, M. E

HATFIELD, M. O

HAYDEN, L. A.

HAYES, R. E.

HAZEN, D. A.

HEIM, L.

HEUTMAKER, M. S.

HIESTER, A. E.

HILL, D. A.

HILL, D. A.

HILL, J. R.

$9,10,12-15,17,20,32,33,36-44,46,48,50,52-56,58,64,89,107,110,124-127$

HINKEN, J.

HOER, C. A

HOFFMAN, J.

$83-97,127,129$

HOLLMANN, $\mathrm{H}$

HOLLOWAY, C. L

HOLMLARSEN, F.

HOLT, D. R.

HONDA, T.

HUANG, D. X.

HUANG, Q.

HUDSON, P. A.

HUNTER, J. D.

HUNTER, R. D.

HUNTLEY, L. E.

JACOBSON, M. D.

119

JAEGER, N. A. F.

$\ldots \ldots \ldots 118,119$

76,80

JANEZIC, M. D.

JARGON, J. A.

$71,72,88,92-94,96-98$ 
JARVIS, J. R.

JESCH, R. L.

JICKLING, R. M.

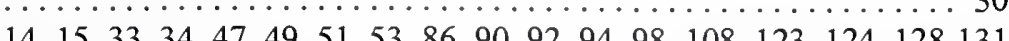

JOHNK, R. T.

JOHNSON, R. B.

...................................

$112,124,128,129$

$36,37,39-42,110$

JONES, C. A.

JONES, R. N.

$27-30$

JUDISH, R. M.

$34,81,87-89,94,97,98,108,126,127$

JUROSHEK, J. R.

$78,82,85-87,123$

KAISER, R. K. F.

$74,75,78,81,83,85-87$

KAMPER, R. A.

$88,90,91$

KANDA, M. . .

KANTOR, Y.

$13,14,16-18,20-26,36-61,63-68,70-72,93,104-106,110,111,113,115,129,132,133$

KATO, $\mathrm{H}$.

KENT, B.

75

KENT, B. M.

KERNS, D. M.

KESSEL, W.

KNNG, J.

KNNZEL, G. A.

KISSICK, W. A .

$19,25,26,98,129$
$\ldots \ldots 100,101$

KLEMPERER, W. K.

$19,25,26,98,129$
$\ldots \ldots 100,101$

KNOPP, K. J.

KOCH, M. E.

107,11

KODALI, V.P.

KOEPKE, G. H.

KOMAREK, E. L.

KREMER, D. P.

KRUPKA, J.

KU, H. H.

127

KUFFEL, J.

KUHN, M.

KUKOL, R. F.

. 37

$13,15,36-39,41,42,44-46,49-53,55,56,58-61$

$89,91,92,94$

$6-11,15,16,18,20$

KWALKO, S. F.

27-30

LADBURY, J. M.

LARSEN, E. B.

LARSEN, N. T.

LARSON, T. R.

. 123

LARSON, W.

53,124

LAUBER, W.

LAWTON, R. A.

. 30

133

LEMANCYZYK, J

$36,37,43,44,46,48,49$

LEWIS, R. L.

LITTLE, W. E

LIU, B. H.

$13,15,24,26,42-46,50-52,54,56,57,62,63,67-69,72,130$

LIVINGSTON, E. M.

$84,85,91,92,124,128,129$

LLOYD, F.

$94,96,98$

LOUGHRY, T. A

$4,96,98$

LOWE, L. T.

LOWELL, W. R.

$21,33,34,53,66,73,97,111,113-119,124,127-129$

LUFT, P. E.

LYONS, R. M.

MA, M. T.

MA, Q. V.

MACREYNOLDS, $\mathrm{K}$

MADSEN, W. B.

$5-12,19,20,23,27,125,132$

MAJOR, J. R.

$34,93,129$

....... 59

$83,84,86,87$

127

.... 37

130,132

121,122

$17-19,21,23,38-50,52-54,56-65$ 
MAJUMAR, A.

MALEWSKI, R.

53,124

MANNEY, C. H.

$93,95,131$

MANTESE, J. F.

$27,29,30$

MARKS, R. B.

$74-83,110,122$

MARLER, F. E.

MARTZLOFF, F. D

MASCLET, G.

50

MASTERSON, K. D

MAY, W. G.

$\ldots \ldots \ldots \ldots \ldots 133$

$36,40-42,44,45,50,76,121$

$93,129,133$

MCCAA, W. D. Jr.

119,120

MCFARLANE, E. J.

$\ldots 10$

MCINERNEY, $M$.

MCKENNA, $P$

MCLAUGHLIN, R. G.

MEDLEY, H. W

51,111

MELQUIST, D. G.

$18,41,45,47,48,61,71,72,94$

MENSA, D. L

METZKER, R. F.

MEYER, $\mathrm{K}$.

MICHELI, A. L.

MIERS, T.

MILLER, C. K. S.

82

MILLIGAN, T.

$58,60,62,64,66,87,102,105,123,124,126,130$

MODELSKI, J.

MONTGOMERY, J. P.

MORA, P.

MORGAN, J. M.

MORGAN, W. L.

MORRIS, D.

MUTH, L. A.

$3-10,12,14-16$

NAHMAN, N. S

NELSON, R. E.

NEWELL, A. C.

NORGARD, J. D

NORGARD, J.D.

NOVOTNY, D. R

OLYSLAGER, F.

$54,56,72,95,112-120,127,130$

ONDREJKA, A. R.

ONO, R. H.

ORR, R. D.

$19,97,124,128,132$

4-7, 9-19, 24-26, 71

$3-5,47$

OSSOLA, P.

PAGE, J. M.

PAO, C. K.

PARNELL, W.

$\ldots \ldots 75$

$18,21,36,37,39-42,47,48,57,58,61,66,71,72,94,97,98,110,111,113$

PEISER, H.S

75,121

$13,14,16,41,42,44,45,47,51,55,57,60,61$

PERERA, S.

PERSINGER, R. R.

PESTA, A.

PETERSEN, J. D.

PETERSON, R.

$13,100,102$

PHILLIPS, K. R.

PIETRUSZKO, S.

PINKASI, M.

PORTIER, G.

PRICKETT, M. J. 
PUCIC, S. P.

RAKOSKI, $\mathrm{B}$.

RALSTON, A.

RANDA, J.

$37-43,45,47-52,55-57,74,99$

RANDA, J.

REBULDELA, G.

REEVE, G. R.

REIDY, A. R.

REPJAR, A. G.

RETTIG, J. B.

$37-43,45,47-52,55-57,74,99$
$\ldots \ldots \ldots \ldots \ldots \ldots \ldots 38$

RIAD, S. M.

$76,79,80,89$

RICHMOND, J. H

RIDDLE, B. F.

RIES, F. X.

$19,20,46,53,56,58,61-63,69,72,81,82,85-87,89,106,121,122,127$

RISLEY, A. S.

ROE, K. C.

ROGERS, E. H.

ROGERS, G. J.

ROGERS, J. E.

ROSE, C.

$4,7,8,10-14,17-20,24,107$

ROSHKO, A.

$111,113,117$

ROTH, R. S.

$\ldots \ldots 34$

RUDMAN, D. A.

RUMFELT, A. Y.

RUSSELL, D. H.

RUSYN, T.

$7,29,37,39-41,43,44$

SANGSTER, R. C. $59-63,65,89,92,126$

SANTORO, A.

$92,93,108,124$

SAULSBERY, L. F.

SAWYER, D. E.

SCAVENNEC, A.

SCHAPPACHER, J. B.

SCHAPS, S. R.

SCHRANK, H. E.

SCOTT, W. W., Jr

SEGA, R. M.

SEIFERT, M.

SELBY, M. C.

SENGUPTA, L.

75,121

SENGUPTA, S.

SEQUEIRA, H. B.

SHAFER, J. F.

SHEERAN, $S$.

... 29

75,121

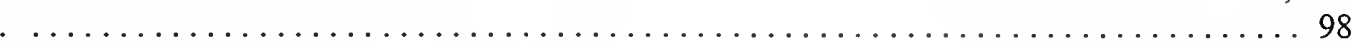

$83,84,98$

SHERWOOD, G. V.

$128,129,131$

SHIH, Y. C.

85,96

. 129

118,130

SHIMODA, R. Y

SHIUE, J. C.

SHOAF, J. H.

. 76

SIMPSON, P. A.

SINDT, C. F.

SKINNER, J. P.

SLADEK, M. J.

SMART, G. R.

SMITH, R. B.

SORGNIT, J.

$22,57,67-69,128,129$

15,56

79,82 
SPIESS, W.

SPLETT, J. D.

SREENIVASIAH, I.

STAFFORD, R. B.

STEPPE, M. C. .

STILES, J. A.

STOKES, R.W.

109

STOLTENBERG, R. E. .

$109,124,128-131$

STORK, F.J.B.

STUBENRAUCH, C. F.

$3-5,7,11,12,14,16,18-22,60$

STWALLEY, W. C.

SUGAR, G. R.

SUTTON, D. D.

TAGGART, H. E.

TAMURA, D. T.

TAUSCH, H. J.

$19,22,34,60,62,67,73,132$

TERRELL, L. A.

$4-7,12$

THOMAS, C. L.

TIPPET, J. C.

TOBAR, M.

TOFANI, S.

TOMOEDA, N. C.

$37,41,110,111,113$

TRABELSI, A.

TREADO, M. J.

TRYON, P. V.

TUTTLE, J. D.

VAN HEESWIJK, R.

VANDERAH, T. A.

VANZURA, E. J.

VECCHIA, D. F.

VERMA, K. K.

VOGLER, L. E.

VORIS, P. C.

WACKER, P. F.

WAINWRIGHT, A. E

WAIT, D. F.

WAIT, J. R.

WAKEFIELD, J. P.

WALKER, D. K.

WEIDMAN, M. P.

WEIL, C. M.

WEITZEL, D. H.

WEN, C. P.

WERNER, P. E

. 71

\section{7}

\section{9} 94

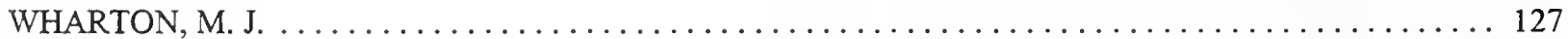

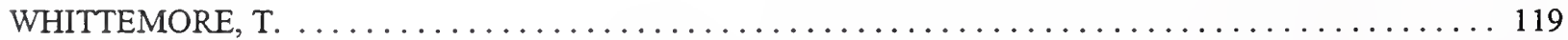

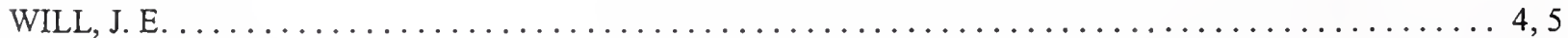

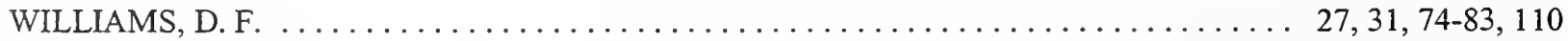

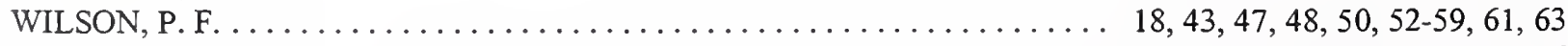

WILSON, W. A.

WITTMANN, R. C. . . . . . . . . . . . . . . . . . . . . . 3-12, 14, 16, 108, 110, 121, 124

WONG-NG, W.

WOODRUFF, J. J. . . . . . . . . . . . . . . . . . . . . . . . . . . . . . . . 22

WORKMAN, J. L. . . . . . . . . . . . . . . . . . . . . . . . . . . . 19, 64-67, 132

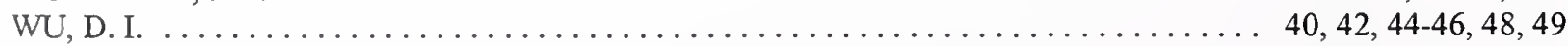

WU, Y. .

WYSS, J. C.

$15,16,18,51,56,57,61,127$ 


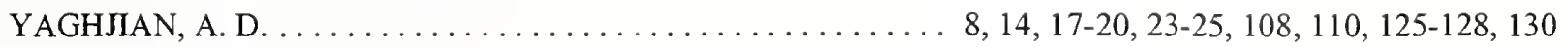

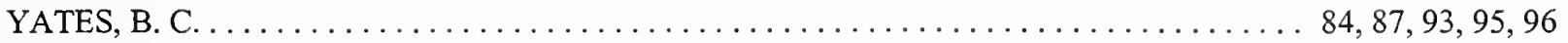

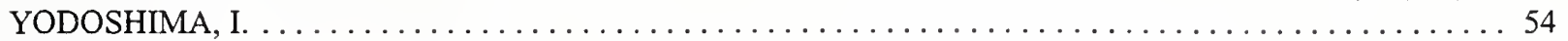

YOUNG,

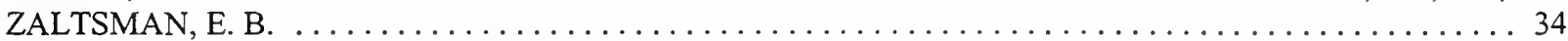

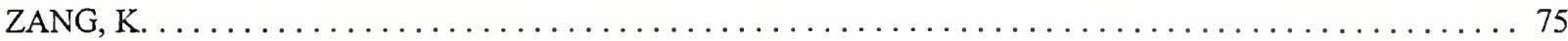

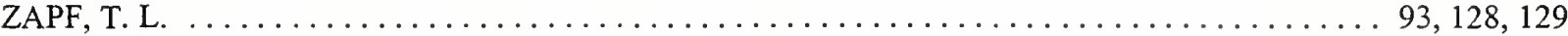

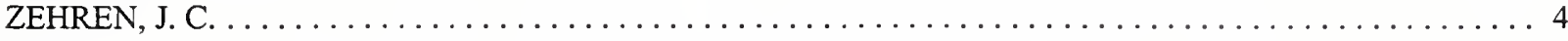




